\title{
Ion mobility-mass spectrometry for structural characterization and applied 'omics: A study in neurodegenerative diseases
}

James Russell Arndt III

Follow this and additional works at: https://researchrepository.wvu.edu/etd

\section{Recommended Citation}

Arndt III, James Russell, "Ion mobility-mass spectrometry for structural characterization and applied 'omics: A study in neurodegenerative diseases" (2015). Graduate Theses, Dissertations, and Problem Reports. 5123.

https://researchrepository.wvu.edu/etd/5123

This Dissertation is protected by copyright and/or related rights. It has been brought to you by the The Research Repository @ WVU with permission from the rights-holder(s). You are free to use this Dissertation in any way that is permitted by the copyright and related rights legislation that applies to your use. For other uses you must obtain permission from the rights-holder(s) directly, unless additional rights are indicated by a Creative Commons license in the record and/ or on the work itself. This Dissertation has been accepted for inclusion in WVU Graduate Theses, Dissertations, and Problem Reports collection by an authorized administrator of The Research Repository @ WVU.

For more information, please contact researchrepository@mail.wvu.edu. 
Ion mobility-mass spectrometry for structural characterization and applied 'omics: A study in neurodegenerative diseases

\author{
by \\ James Russell Arndt, III \\ Dissertation submitted to the \\ Eberly College of Arts and Sciences \\ at West Virginia University \\ in partial fulfillment of the requirements \\ for the degree of \\ Doctor of Philosophy \\ in \\ Chemistry
}

\author{
Approved by \\ Stephen J. Valentine, Ph.D., Committee Chairperson \\ Justin Legleiter, Ph.D. \\ Lisa Holland, Ph.D. \\ Suzanne Bell, Ph.D. \\ David Smith, Ph.D.
}

C. Eugene Bennett Dept. of Chemistry

Morgantown, West Virginia

2015

Key Terms: ion mobility, mass spectrometry, hydrogen-deuterium exchange, electrospray ionization, huntingtin protein, flanking sequences, neuropathy, proteomics, metabolomics, lipidomics

Copyright 2015 James Russell Arndt, III 


\section{ABSTRACT \\ Ion mobility-mass spectrometry for structural characterization and applied 'omics: A study in neurodegenerative diseases}

by

\section{James Russell Arndt}

Huntington's disease is neurodegenerative disease caused by an expanded polyglutamine-coding CAG repeat in exon 1 of the huntingtin gene. Huntingtin exon 1 forms the primary toxic amyloid structure in Huntington's disease; disease severity is directly correlated with polyglutamine length. Recent works have shown that fully formed amyloid plaques may not represent the most toxic species in Huntington's disease; the most neurotoxic species may be small, diffuse oligomer (4 - 20 monomer units) that are precursors to amyloid plaques. While the polyglutamine region is undisputed as the primary constituent of amyloid structure, aggregation kinetics and morphology are regulated by the presence of flanking sequences that are $\mathrm{N}$ - and $\mathrm{C}$ terminal to theamyloid forming tract. The first seventeen residues of huntingtin exon 1 (Nt17) can form an amphipathic $\alpha$-helix depending upon solution conditions and the presence of a binding partner, and in most cases, mediates oligomer formation. Cterminal to the polyglutamine tract is a proline-rich region, or in the case of a model peptide a polyproline region (polyP), that can form a polyproline-type II (PPII) helix, which may regulate Nt17 in huntingtin protein with short polyglutamine regions. Much is unknown regarding residue-specific Nt17-Nt17 and Nt17-polyP interactions. The work described here utilized state-of-the-art deuterium exchange mass spectrometry techniques to identify critical hydrophilic residues in early stages of oligomer formation. Monomeric and multimeric conformations of $\mathrm{Nt17}$, idependent og the polyglutamine 
domain, were then studied using ion mobility-mass spectrometry and molecular dynamics to gain insight into the earliest stages of Nt17-Nt17 association, and thus, aggregation. Monomeric and multimeric Nt17 could form extended helices in the gas phase. Key hydrophilic residues were chemically modified, which resulted in a sharp decline in multimer formation. Finally, Nt17-polyP interactions were probed using gasphase deuterium exchange mass spectrometry, supplemented with molecular dynamics and an exchange kinetics model. The obtained gas-phase structures showed a reduction in Nt17 extended a-helix, when compared to a monomeric and extended homodimeric conformation. Thus, it is hypothesized that polyP regulates Nt17 by not allowing transition to the amphipathic $\alpha$-helix. The results of this study examine the structural heterogeneity of a sequence thought to drive a potentially toxic aggregate morphology, pinpoint key residues in early oligomer formation, and provide strategies for regulation of oligomer formation. 


\section{DEDICATION}

For Laura.

"No problem that is describable is unsolvable."

-Rick Laborde, November 28, 2014 


\section{ACKNOWLEDGEMENTS}

I would like to express my sincerest gratitude toward every individual who has served as a mentor over my graduate career: Suzanne Bell, for getting me started on my path; Justin Legleiter, for providing hours of meaningful insight, conversation, and direction about an extremely complex system; and, most importantly, Steve Valentine for being one of the most patient, honest, and helpful individuals I have ever had the pleasure of working for. If not for these people, I would not be the scientist I am today.

I would also like to thank the rest of my committee, Dr. Lisa Holland and Dr. David Smith, for valuable insight and guidance over the last several years. Your time and effort is greatly appreciated.

To my lab mates, Hossein Maleki, Samaneh Kondalaji, Mahdiar Khakenejad, Megan Maurer, and Greg Donohoe, you are family. You have all been a part of the greatest journey in my life, and I could not have asked for a better group of people to spend it with. I would also like to thank Dr. Nicole Shamitko-Klingensmith, Maxmore Chaibva, Xiang Gao, and Albert Pilkington for allowing a lowly mass spectrometrist to crash your weekly atomic force microscopy party.

A large thank you is also due to all of the undergraduate assistants that have helped in all facets of my work: Robert Brown, Arlo Parker, and Olivia Sarver. You were all essential to a majority of this work, and it would not have gone as smooth as it did without you. 


\section{TABLE OF CONTENTS}

Abstract

Dedication

Acknowledgements $\quad$ v

Table of Contents $\quad$ vi

List of Figures $\quad$ xi

List of Tables $\quad$ xiii

List of Symbols and Abbreviations $\quad$ xiv

1. Introduction: Insights to neurodegenerative disease provided 1 by deuterium exchange and ion mobility-mass spectrometry

1.1 Huntington's disease oligomerization is facilitated by the 1 presence of flanking sequences

1.2 The amphipathic a-helix 3

$1.3 \mathrm{Nt} 17$ in the oligomerization pathway 5

1.4 Targeting Nt17 for HD remediation $\quad 7$

1.5 Mass spectrometry for structural analysis 10

1.5.1 Electrospray ionization 10

1.5.2 Hydrogen-deuterium exchange $\quad 11$

1.5.3 Covalent labeling for residue-specific structural 15 interrogation

1.6 lon mobility-mass spectrometry 16

1.6.1 Ion mobility fundamentals 16

1.6.2 Ion mobility-mass spectrometry in bioanalysis and $\quad 19$ amyloid study

1.6.3 Gas-phase deuterium exchange in IMS-MS 21 
2. Lysine Residues in the N-Terminal Huntingtin Amphipathic $\alpha-$ Helix Play a Key Role in Peptide Aggregation

2.1. Introduction: The case for deuterium exchange MS in HD characterization

2.2 Materials and methods

2.2.1 Sample preparation

2.2.2 Thioflavin T assay

2.2.3 Atomic force microscopy

2.2.4 DHX Tandem mass spectrometry

2.2.5 DHX Data Analysis

2.3 Results and discussion

2.3.1 Htt peptide predominantly forms fibrillar aggregates under experimental conditions

2.3.2 Full MS of Labeled $\mathrm{Nt}_{17} \mathrm{Q}_{35} \mathrm{P}_{10} \mathrm{~K}_{2}$ demonstrates six distinct charge states.

2.3.3 Protein HDX and DHX exchange levels

2.3.4 Deuterium uptake by residue - H-to-D-exchange (HDX) demonstrates deviation from maximum uptake

2.3.5 Deuterium protection by residue - D-to-H-exchange (DHX) demonstrates protection in Nt17

2.3.6 HDX and DHX by residue show increased protection on lysine residues

2.3.7 Comparison of the structural findings 
3. Huntingtin $\mathrm{N}$-terminal monomeric and multimeric structures destabilized by covalent modification of heteroatomic residues

3.1. Introduction: Nt17 interactions and modifications studied by IMS-MS

3.2 Materials and methods

3.2.1 Sample preparation

3.2.2 Carbethoxylation with diethylpyrocarbonate (DEPC)

3.2.3 Ion Mobility Spectrometry-Linear Ion Trap Mass Spectrometry (IMS-MS).

3.2.4 Molecular dynamics

3.3 Results and discussion

3.3.1. Nt17 adopts several monomeric and multimeric conformations

3.3.2 Compact and elongated $\mathrm{Nt} 17[\mathrm{M}+2 \mathrm{H}]^{2+}$ ion structures from molecular dynamics simulations

3.3.3. Conformations of multimeric ions

3.3.4 Carbethoxylation introduces a new monomer structure

3.3.5 Lysine- 6 is modified at elevated DEPC concentrations

3.3.6 Interpretation of structural findings

3.3.7 Covalent modifications alter structures 
4. Polyproline peptides interact with $\mathrm{Nt} 17$ and suppress huntingtin aggregation

4.1. Introduction: PolyP as a regulator of Nt17 101

$\begin{array}{ll}\text { 4.2 Materials and methods } & 104\end{array}$

4.2.1. Sample preparation 104

4.2.2. Ion mobility - mass spectrometry 104

$\begin{array}{ll}\text { 4.2.3 Complex energetics } & 105\end{array}$

4.2.4 Gas-phase hydrogen-deuterium exchange (GP-HDX) 106

4.2.5 Molecular dynamics simulations 106

4.2.6 Hydrogen accessibility scoring and kinetics model 107

$\begin{array}{ll}\text { 4.2.7 Purification of htt exon } 1 \text { construct } & 108\end{array}$

$\begin{array}{ll}\text { 4.2.8 Atomic force microscopy } & 109\end{array}$

$\begin{array}{ll}4.3 \text { Results and discussion } & 110\end{array}$

4.3.1. Nt17 and polyP form two stable heterodimeric 110 complex conformers

4.3.2 Heterodimer conformations vary in deuterium uptake 115

4.3.3. Gas-phase Nt17-polyP heterodimer structure 118

4.3.4 Incubation with polyP reduces htt exon 1 fibril 121 formation

4.3.5 Interpretation of structural findings 124

$\begin{array}{ll}4.4 \text { References } & 128\end{array}$

5. Outlook: Physiologically relevant post-translational modifications of $\mathrm{Nt17}$ and regulation through complexation

5.1. Work in progress: Acetylation of $\mathrm{Nt}_{17} \mathrm{Q}_{35} \mathrm{P}_{10} \mathrm{KK}$ by sulfoNHSA 
5.1.1. Acetylation of $\mathrm{Nt} 17$ suppresses aggregate formation and alters aggregation kinetics

5.2 Work in progress: K to Q mutations in Nt17 136

5.2.1. $\mathrm{K}$ to $\mathrm{Q}$ mutations alter Nt17 secondary structure 136

5.3 Future direction: targeting Nt17 with other proteins 138

5.4 Concluding remarks 139

$\begin{array}{ll}5.6 \text { References } & 142\end{array}$ 


\section{LIST OF FIGURES}

1.1 Schematic representation of various features of the huntingtin (htt) protein

2.1 The htt peptide $\mathrm{Nt} 17 \mathrm{Q}_{35} \mathrm{P}_{10} \mathrm{~K}_{2}$ readily forms fibrils

2.2 Disaggregation of $\mathrm{Nt}_{17 Q_{35}} \mathrm{P}_{10} \mathrm{~K}_{2}$ by addition of $\mathrm{ACN} / \mathrm{HOAc}(90 / 10)$

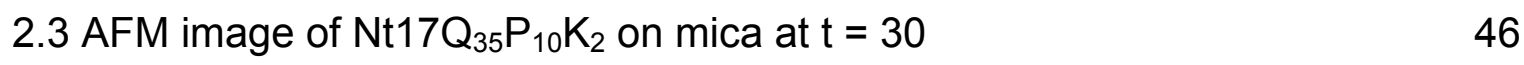

$\begin{array}{ll}2.4 \text { Scrambling controls } & 47\end{array}$

2.5 On-line DHX schematic $\quad 48$

2.6 Full mass spectrum of the unlabeled $\mathrm{Nt}_{17} \mathrm{Q}_{35} \mathrm{P}_{10} \mathrm{~K}_{2}$ peptide shows $\quad 49$ six accessible charge states

$2.7 \mathrm{~N}$-terminal region of $\mathrm{Nt} 17 \mathrm{Q}_{35} \mathrm{P}_{10} \mathrm{~K}_{2}$ peptide is solvent exposed and contains secondary structure in the aggregated form

52

2.8 The Nt17 region is structured early in the aggregation process 57

3.1 False color IMS-MS distributions of Nt17 ions 71

3.2 Extracted $t_{D}$ distributions for dimer, trimer, and tetramer ions $\quad 72$

3.3 Nt17 ribbon structures calculated from MDS 73

3.4 Nt17 dimer stuctures calculated from MDS 76

$3.5 t_{D}$ distributions of covalently modified $\mathrm{Nt} 17[\mathrm{M}+2 \mathrm{H}]^{2+}$ monomer at $\quad 79$ $5 x, 50 x$, and $250 x$ DEPC

3.6 2D IMS-MS distribution showeing an expanded region of unlabeled, singly-, and doubly-modified Nt17 at 5x, 50x, and 250x DEPC concentrations

3.7 2D IMS-MS distributions of modified dimer species

3.8 Mobility selected MS/MS analysis of the intermediate, doublymodified conformer

4.1 Expanded regions of representative IMS-MS distributions 
4.3 Deuterated IMS-MS distributions 116

$\begin{array}{ll}\text { 4.4 Deuterium uptake by species } & 117\end{array}$

4.5 Nt17-polyP heterodimer gas-phase structures 118

4.6 Representative AFM images 122

5.1 Acetylation of $\mathrm{Nt}_{17} \mathrm{Q}_{35} \mathrm{P}_{10} \mathrm{KK}$ reduces $\beta$-sheet formation 134

5.2 MS and IMS-MS distributions of Nt17 K6Q point mutations 137

5.3 Schematic of Nt17-mediated htt exon 1 aggregation for modified 140 and regulated $\mathrm{Nt} 17$ 


\section{LIST OF TABLES}

2.1 Total deuterium uptake and retention by charge state 50

3.1 Calculated collision cross-sections for selected Nt17 modified and $\quad 80$ unmodified conformers

4.1 Calculated collision cross sections for all relevant species

113 


\section{LIST OF SYMBOLS AND ABBREVIATIONS}

$\begin{array}{ll}\text { ACN } & \text { acetonitrile } \\ \text { HOAc } & \text { acetic acid } \\ \mathrm{NH}_{4} \mathrm{OAc} & \text { Ammonium acetate } \\ \mathrm{HDX} & \text { hydrogen-deuterium exchange (H-to-D exchange) } \\ \text { DHX } & \text { deuterium-hydrogen exchange (D-to-H exchenge) } \\ \text { ESI-MS } & \text { electrospray ionization mass spectrometry } \\ \text { ETD } & \text { electron transfer dissociation } \\ \text { AFM } & \text { atomic force microscopy } \\ \text { IMS-MS } & \text { lon mobility-mass spectrometry } \\ \text { Nt17 } & \text { N-terminal 17-residue amphipathic } \alpha \text {-helix of huntingtin } \\ \text { MDS } & \text { Molecular dynamics simulations } \\ \text { DEPC } & \text { diethyl pyrocarbonate } \\ t_{D} & \text { drift time } \\ \text { PolyP } & \text { Poly-proline } \\ \text { PolyQ } & \text { Poly-glutamine } \\ \text { NHSA } & N \text {-Hydroxysuccinimide } \\ & \end{array}$




\section{Introduction: Insights to neurodegenerative disease provided by deuterium exchange and ion mobility-mass spectrometry ${ }^{1}$}

${ }^{1}$ Reproduced in part with permission from BioMolecular Concepts: The emerging role of the first 17 amino acids of huntingtin in Huntington's disease. Arndt, J. R., Chaibva, M., and Legleiter, J. BioMolecular Concepts 6, 2015, 33-46.

\subsection{Huntington's disease oligomerization is facilitated by the presence of flanking sequences.}

Huntington's disease (HD) is a fatal neurodegenerative disease caused by an expanded polyglutamine- (polyQ) coding CAG repeat domain in exon 1 the huntingtin gene (HTT). ${ }^{1}$ The expanded polyQ domain in the resulting huntingtin protein (htt) forms amyloid fibrils and inclusion bodies in vivo, leading to distinct symptoms of neurodegeneration, such as chorea, fatigue, changes in personality, loss of motor function, and eventually, death. ${ }^{2}$ Disease length threshold for the polyQ repeat is approximately 35 residues; disease severity is directly correlated to poly $Q$ length, while age of onset is inversely correlated. ${ }^{3}$ Currently, there is no cure for HD.

Recent research efforts have been focused on structural characterization of in vitro polyQ fibril structure $;^{4-7}$ however, fibrils may represent a neuroprotective species. Formation of inclusion bodies can be a predictor of cell survival. ${ }^{8}$ Oligomers, small soluble aggregates composed of four to twenty monomer units, could represent the most toxic species in HD. In the same study that showed prediction of cell survival as a function of inclusion body formation, the presence of diffuse oligomeric species almost always led to neuronal death. ${ }^{8}$ $\mathrm{Htt}$ oligomers have been observed by time-dependent electron microscopy in 
human HD brains exhibiting a phenotype for disease. ${ }^{9}$ Flanking sequences that surround the polyQ domain drive htt oligomerization. C-terminal to the polyQ tract is a proline-rich domain that contains a PPII helix (Figure 1.1). This tract supports anchoring and subsequent aggregation on lipid substrates. ${ }^{10}$

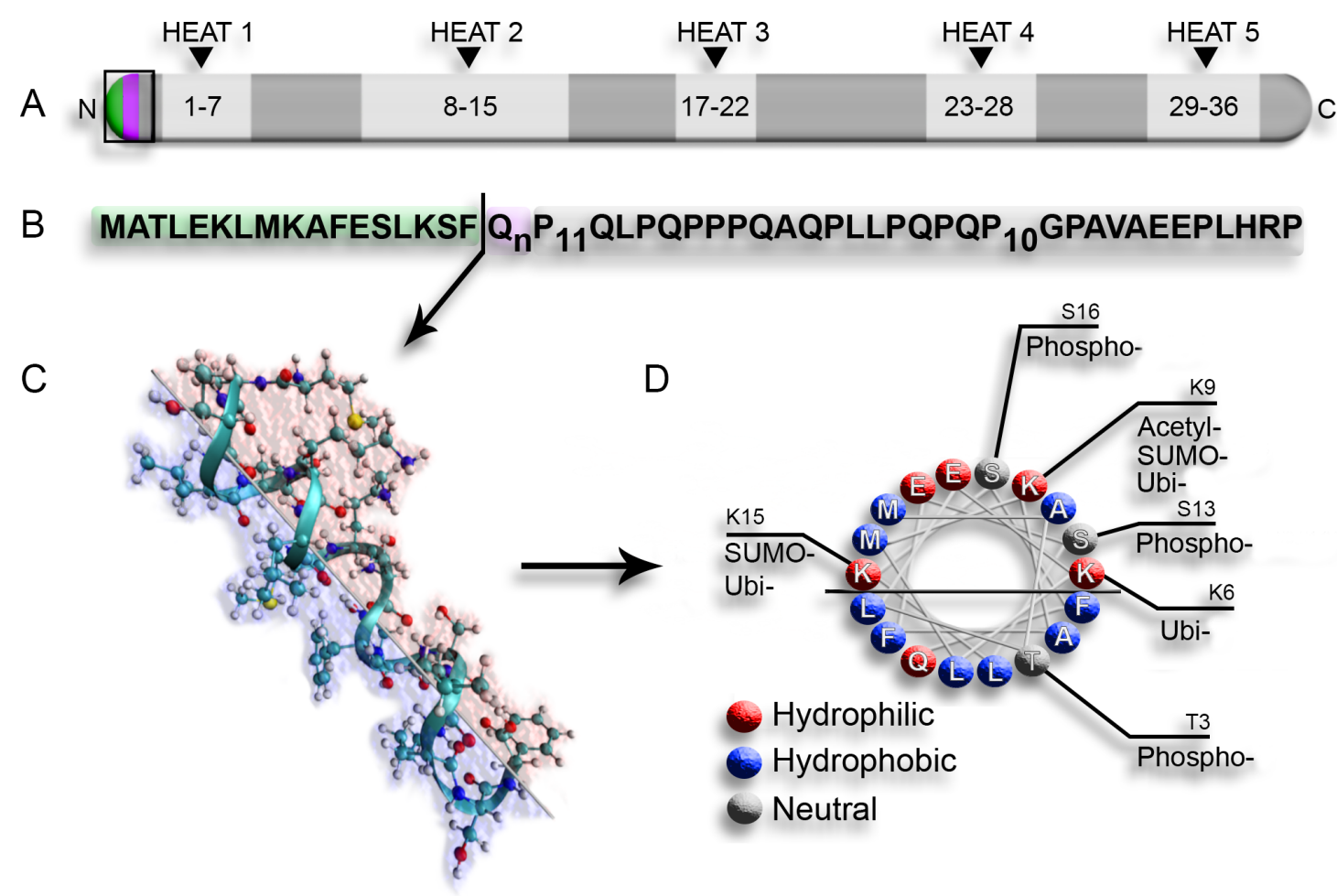

Figure 1.1. Schematic representation of various features of the huntingtin (htt) protein. (A) Full-length htt with HEAT repeat sites (dark gray). Exon 1 is denoted by the black box. (B) Primary sequence of htt exon 1 indicating Nt17 (green), polyglutamine (polyQ) (purple), and the proline-rich region (gray). (C) Theoretical three-dimensional structure of Nt17 indicating hydrophilic (red) and hydrophobic (blue) faces of an amphipathic $\alpha$-helix. (D) View down the barrel of the $\alpha$-helix showing relative hydrophobicity of each residue (same color scheme as panel C), as well as the sites of reported posttranslational modification. Reproduced from BioMolecular Concepts: The emerging role of the first 17 amino acids of huntingtin in Huntington's disease. Arndt, J. R., Chaibva, M., and Legleiter, J. BioMolecular Concepts 6, 2015, 33-46.

$\mathrm{N}$-terminal to the poly $\mathrm{Q}$ region is a seventeen-residue tract (Nt17) that can form an amphipathic $\alpha$-helix once incorporated into an amyloid fibril. ${ }^{4,11}$ This domain is thought to be required for htt oligomer formation both in solution ${ }^{12}$ and on a lipid substrate. ${ }^{10}$ Since Nt17 presents a potential driving force behind the formation of 1. Introduction: Insights to neurodegenerative disease provided by deuterium exchange and ion mobility-mass spectrometry 
the most toxic aggregate species, it represents a potential target for therapeutic remediation of HD. Thus, a thorough understanding of the nature of amphipathic a-helices and interactions of Nt17 is vital.

\subsection{The Nt17 amphipathic $\alpha$-helix.}

An amphipathic $\alpha$-helix consists of a typical $\alpha$-helix whose faces are separated into a hydrophobic and hydrophilic domain (Figure 1.1C). This arrangement is suitable for hydrophobic interaction with other helices in an aqueous cellular environment, ${ }^{13,14}$ as well as lipid membrane binding. ${ }^{15,16}$ Amphipathic helices preferentially bind to sites of membrane curvature and can be used as sensors of curved membranes. ${ }^{17} \mathrm{Nt} 17$ has been shown to sequester truncated htt exon 1 peptides to regions of curvature on supported lipid bilayers. ${ }^{18}$ The Nt17 primary sequence contains three basic lysine residues at positions 6,9 , and 15 . Residues 6 and 9 lie on the boundary of the hydrophilic and hydrophobic faces in a theoretical full helix (Figure 1.1C and D). Additionally, two methionine, two serine, and two glutamic acid residues are encompassed in the hydrophilic face. The hydrophobic face consists of two phenylalanine residues, three leucine residues, and an alanine residue, with a leucine and a phenylalanine at each side of the boundary. It would appear that residues in the boundary region are critical in lipid membrane binding and intermolecular interaction. Simulation of lipid membrane association shows K6 and K15 are strong hydrogen bond donors with lipid bilayers. ${ }^{19}$ These studies point to critical 
interactions involving amphipathic helices that can potentially modulate toxicity and aggregation kinetics.

While there is little doubt Nt17 forms an amphipathic helix, whether the monomeric form of Nt17 is helical, either by itself or attached to the polyQ domain, has been the topic of much discussion. The first crystal structure of htt exon 1 showed that Nt17 is fully helical, with the helix extending into the polyQ domain. ${ }^{20}$ Atomistic simulation in explicit aqueous solvent, circular dichroism spectropolarimetry (CD) and solution NMR also point to transient helical properties in the monomeric form. ${ }^{21-24}$ Separate analytical untracentrifugation (AUC) and CD studies have shown that Nt17 alone is only transiently helical, and only forms the full, stable amphipathic helix upon association with a binding partner. ${ }^{12}$ Jayaraman et al. determined by the methods listed above (AUC and $\mathrm{CD}$ ) that mostly random coil monomeric Nt17 exists in solution in equilibrium with an $\alpha$-helix rich tetrameric species that can nucleate amyloid formation. ${ }^{12}$ The experiment used Nt17 with short polyQ segments to model the early stages of amyloid formation. The AUC experiment showed the propensity for $\mathrm{Nt17}$ to tetramerize, while concentration-dependent CD showed an increase in a-helicity for isolated Nt17 aggregates. ${ }^{12}$ Combined, these peptide experiments provide quaternary arrangement and associated secondary structure. Those that did not tetramerize are hypothesized to serve as a pool from which monomer addition can occur. Solid state NMR and FTIR studies confirm that the Nt17 $\alpha$-helix is conserved upon aggregation with polyQ segments above $n=30 .{ }^{11}$ The FTIR experiments showed a strong IR absorbance at $1655 \mathrm{~cm}^{-1}$, indicative of $\alpha$-helical 
character, from both small aggregates of $\mathrm{Nt17}$ as well as htt exon 1 model peptides. Additionally, ssNMR chemical shifts indicate residues $4-11$ are in an a-helical arrangement, while residues $17-19$ have a clear $\beta$-sheet structure. ${ }^{11}$ The disordered monomeric structures reported by Jayaraman et al. are in contrast to other studies that state Nt17 is mostly helical in solution. Kelley, et al. show from atomistic simulation that $\mathrm{Nt17}$ exists in a two-helix bundle at $300 \mathrm{~K}$ and transiently exists as a straight helix. ${ }^{17,24}$ Their study shows Nt17 tends toward a more globular state at temperatures exceeding $400 \mathrm{~K}$. Their findings could corroborate the structural heterogeneity reported in the AUC and CD studies, though, with regard to secondary structure, they are in disagreement. Still, compelling evidence suggests the $\alpha$-helix is conserved in the aggregated form and located on the periphery of the amyloid structure, ${ }^{5,11}$ which can have significant ramifications in aggregation mechanism.

\section{$1.3 \mathrm{Nt17}$ in the oligomerization pathway.}

$\mathrm{Nt} 17$ has been implicated in driving the initial phases of htt exon 1 interaction. ${ }^{11,25-28}$ As such, several mechanisms exist that are mediated by Nt17. Most agree that the initial phase of aggregation begins with interaction of $\mathrm{Nt17}$, which forms a population of small, $\alpha$-helix rich oligomers ${ }^{23,29,30}$ that are widely considered to be the toxic species in Huntington's disease ${ }^{9,31}$ and this represents the rate-limiting step in htt exon 1 aggregation. ${ }^{12,32}$ That is, in a cellular environment, pathogenic htt exon 1 oligomer population did not appreciably change despite recruitment of moomers into inclusion bodies, hence, the 
formation of oligomeric species is the rate-determining step in one aggregation pathway, most likely one that is mediated by Nt17. ${ }^{32}$ PolyQ peptides that do not have the $\mathrm{N}$-terminal flanking sequence do not form oligomeric intermediates and as a result, proceed directly to fibrillar aggregates. ${ }^{12,26}$ The mechanism for $\mathrm{Nt} 17$ mediated htt exon 1 aggregation begins with an association between monomeric htt exon 1 that is rich in $\alpha$-helix structure. As stated, this helicity may be a result of, ${ }^{12,26,33}$ or preclude, ${ }^{22,24}$ association. These helical bundles represent the potentially toxic oligomer structure, wherein the polyQ domain is still disordered. ${ }^{12,26}$ The oligomerization process is spontaneous and reversible, with multimer dissociation occurring when polyQ $\beta$-sheet nucleation does not occur, thus replenishing the monomer pool. ${ }^{12,26,33}$ The helical bundle structure places the pathogenic polyQ tract in favorable proximity to form increased $\beta$-sheet structure. ${ }^{11,12,26,33}$ The fibril increases in size by monomer addition, forming elongated $\beta$-sheet rich amyloid fibrils. The fate of $\mathrm{Nt} 17$ appears to be on the periphery of the amyloid structure, as shown by deuterium exchange ssNMR ${ }^{11}$; however, pre-aggregated htt exon 1 does not bind to lipid membranes, which suggests Nt17 is tightly bound, or otherwise unavailable, in the final amyloid structure.$^{34}$ Other mechanisms of htt exon 1 aggregation suggest that amyloid formation is a result of misfolded polyQ $\beta$-hairpin turn that leads to nucleation; in their study, Kar et al. show that an induced $\beta$-turn in polyglutamine repeats, by addition of a Pro - Gly sequence, can induce amyloid formation similar to htt exon $1 .{ }^{35}$ These structures do not, however, contain the Nt17 tract and represent a mechanism of fibril formation that is not mediated by $\mathrm{Nt} 17$, but which does 
explain the polyQ length dependence on aggregation: longer poly $Q$ lengths can experience more structural freedom and are more likely to form a nucleationenhancing $\beta$-turn. ${ }^{35,36}$ These two mechanisms, while at face value are mechanistically dissimilar, may combine to provide a clearer picture of htt exon 1 aggregation. ${ }^{9}$ Variant monomeric structures could lead to the formation of inclusion bodies or annular aggregates. ${ }^{9}$ Monomers can misfold, e.g., formation of a $\beta$-hairpin in monomers with pathogenic polyQ length, and proceed directly to fibrillar aggregates, eventually maturing into inclusion bodies, entirely bypassing the oligomeric intermediate state. Alternatively, Nt17-mediated nucleation occurs via the oligomeric intermediate, which can produce amorphous aggregates that do not resemble amyloid structure, ${ }^{9}$ or undergo a random coil to $\beta$-sheet transition, which leads to fibrillar aggregates and, eventually, inclusion bodies. With several clear pictures of the mechanism by which htt exon 1 forms fibrillar species beginning to emerge, a central goal of htt exon 1 research is to decrease toxicity, either by inhibition of the aggregation process by targeting Nt17 via a chaperone or proteolysis, or forcing the fibrillization pathway toward a non-toxic species through Nt17 modification.

\subsection{Targeting Nt17 for HD remediation.}

Because Nt17 has been implicated in formation of the toxic intermediate, it represents a novel structural target to inhibit aggregate formation, thereby reducing toxicity. The most logical step in controlling Nt17 mediated amyloid formation is the removal of the Nt17 tract all together; a concept that has been 
explored is targeting $\mathrm{Nt} 17$ via proteolysis. ${ }^{33,}{ }^{37} \mathrm{~A}$ tryptic proteolytic fragment of Nt17Q ${ }_{37} P_{10} K_{2}\left(S F Q_{37} P_{10} K_{2}\right)$ exhibited aggregation kinetics and morphologies similar to $\mathrm{K}_{2} \mathrm{Q}_{37} \mathrm{~K}_{2}$, a simple polyQ peptide that lacks both flanking sequences. ${ }^{37}$ Similarly, a $M F Q_{37} \mathrm{P}_{10} \mathrm{~K}_{2}$ tryptic analogue was shown to have similar kinetics to the true tryptic fragment. ${ }^{33}$ In vitro, these studies are simple means of studying Nt17-mediated aggregation, but do not represent a viable means of treatment in vivo, due to the difficulty of selective proteolysis. A means of inhibiting $\mathrm{N}$-terminal aggregation is through structural complexation, either by a chaperone or an antibody-antigen interaction. Interestingly, Nt17 itself has been used to inhibit Nt17-mediated formation of $\mathrm{Nt}_{17} \mathrm{Q}_{37} \mathrm{P}_{10} \mathrm{~K}_{2}$ multimers. ${ }^{26,33}$ In this experiment, aggregation does proceed via a pathway mediated by polyQ nucleation. ${ }^{33}$ The mechanism by which Nt17 inhibits amyloid formation is identical to the mechanism by which initial association occurs, with the caveat that Nt17 alone does not contain an amyloidogenic tract, thereby increasing the distance between adjacent poly $Q$ tracts and increasing the energy required for poly $Q$ aggregation to occur. ${ }^{26}$ Other studies have focused on exogenous protein chaperones to inhibit aggregation. ${ }^{38-40}$ These studies show that the primary mode of aggregation suppression via chaperone interaction is mediation of the misfolded polyQ domain, not Nt17. One of the most promising treatments for neurological disorders stems from the use of antibodies; unlike peptide therapeutics and inhibitors, antibodies are specific only for the target antigen, which, in this case, is htt exon 1 and more specifically, Nt17. Several antibodies exist for htt exon 1 , each recognizing a separate tract. The C-terminal polyP 
domain is a target for antibody $3 \mathrm{~B} 5 \mathrm{H} 10 .{ }^{41,42}$ The antibody MW7 targets a twohairpin structural motif in the polyQ region. ${ }^{41,43}$ Both antibodies have been shown by AFM to disaggregate polyQ amyloid fibrils. ${ }^{41}$ In the past decade, intracellular antibodies, or intrabodies, for Nt17 have been developed that inhibits htt exon 1 fibrillization and cytotoxicity in vitro and cellular huntingtin models. ${ }^{44-46}$ The two intrabodies are C4 ScFv and $V_{L} 12.3$. When cotransfected with htt exon 1 (104Q) into COS-7, BHK-21, and HEK-293 cells, C4 scFv was shown to decrease aggregate formation relative to control groups that were not transfected with the intrabody. ${ }^{46} \mathrm{C} 4 \mathrm{scFv}$ bound to htt exon 1 structures of non-pathogenic length (25Q), as well, which indicates the polyQ conformation does not play a role in intrabody binding, and that the most likely binding target is Nt $17 .{ }^{46}$ The second intrabody, $\mathrm{V}_{\mathrm{L}} 12.3$, also targets the $\mathrm{Nt} 17$ domain; however, unlike $\mathrm{C} 4, \mathrm{~V}_{\mathrm{L}} 12.3$ consists of only a single chain of the typical antibody structure. The overall ability of this antibody to prevent aggregation is highly increased after removal of disulfide bonds within the antibody structure ${ }^{44,45}$ Interestingly, X-ray crystal structure of the antibody-antigen complex revealed that Nt17 was helical in the binding pocket of the intrabody, ${ }^{44}$ which either suggests the binding pocket induces helicity in the proposed random structure of $\mathrm{Nt} 17$, or the intrabody only recognizes helical Nt17. Intrabodies that were co-expressed with a pathogenic GFP-fused htt exon 1 (97 Q) in HEK293 mammalian cells caused a dramatic decrease in huntingtin inclusion bodies relative to controls. The $V_{L} 12.3$ intrabody was at least as effective in retarding aggregate formation as the C4 intrabody, ${ }^{44}$ as shown by fluorescence immunomicroscopy. These studies show that 
structural inhibition of $\mathrm{Nt} 17$ is certainly a viable means of inhibiting aggregation and, potentially, htt exon 1 toxicity.

While many of these studies have targeted Nt17 with some success, none have proposed a residue-specific mechanism for Nt17-driven oligomerization. Aside from ssNMR, which suffers from the requirement to isotopically enrich residues of interest, most of the spectroscopic techniques employed only provide a bulk picture of peptide and protein structure, or only tangentially propose binding faces for $\mathrm{Nt} 17$ self-association and oligomer formation. A structurally heterogenous system like Nt17 requires techniques that can identify specific, coexisting structures and probe interactions in a residue-specific manner.

\subsection{Mass spectrometry for structural analysis.}

\subsubsection{Electrospray ionization.}

Intact protein and peptide analyses were made possible by the advent of electrospray ionization (ESI). ${ }^{47} \mathrm{ESI}$ is a soft ionization technique wherein ions are produced by desolvation of charged droplets from a narrow emitter held at a high potential. ${ }^{47}$ The ionization is performed at atmospheric pressure and in typical cases is not energetic enough to cause fragmentation of proteins and peptides, though at higher ESI potentials some denaturation can occur. ${ }^{48} \mathrm{~A}$ Taylor cone is formed from the liquid flowing through the capillary emitter under the influence of the electric field. A liquid jet emerges toward the counter electrode, producing charged droplets. Due to solvent evaporation, droplets undergo fission as charge density increases. One of two primary ionization mechanisms occurs, depending 
upon size of the analyte. Droplets containing predominantly large molecule analytes, such as large peptides or proteins, undergo several rounds of fission through Coulombic repulsion, and solvent evaporation until only the bare desolvated ion remains (charged residue) ${ }^{49}$ Conversely, smaller analytes, such as small molecule organics or single peptides, can migrate to the surface of the droplet and desorb from the surface, carrying with them residual charge from the droplet surface (ion evaporation). ${ }^{50}$ In either case, ESI produces analytes that may reminiscent of the native state, providing no denaturants are present in the solvents. ${ }^{48,51,52}$ Thus, ESI is most suitable for analyses of intact proteins.

\subsubsection{Hydrogen-deuterium exchange}

Hydrogen-deuterium exchange mass spectrometry has been widely used to characterize protein structure. In solution, HDX is an acid- or base-catalyzed reaction (depending upon $\mathrm{pH}$ ) that replaces amide backbone hydrogens with deuterons in peptide sequences. The mechanism begins with removal of the amide proton by $\mathrm{OD}^{-}$(at base-catalyzed physiological $\mathrm{pH}$ ) followed by deuteration or rehydrogenation depending upon the relative amount of free $D^{+}$ present in the solution ${ }^{53}$. The rate of deuterium exchange is affected by the native folded state and molecular conformation. In the first equation below, $\mathrm{k}_{\mathrm{f}}$ represents fast HDX for exposed backbone amide linkages and ionizable side chains. The second equation represents slower HDX in unexposed, folded species. 


$$
\begin{gathered}
F(H) \stackrel{k_{f}}{\rightarrow} F(D) \\
F(H) \stackrel{k_{1}}{\leftrightarrow} U(H) \stackrel{k_{2}}{\rightarrow} U(D) \stackrel{k_{-1}}{\leftrightarrow} F(D)
\end{gathered}
$$

Backbone hydrogen atoms that participate in hydrogen bonding, such as those within the confines of an $\alpha$-helix, exchange much slower than hydrogen atoms that are not involved in a secondary structure stabilization.

Several studies have shown that structural interrogation is possible through the selective labeling of side-chain heteroatoms with deuterium atoms followed by gas-phase electron transfer dissociation (ETD) ${ }^{54}$ in mass spectrometry ${ }^{55,56}$. Pan et al. utilized gas-phase HDX with deuterated ammonia on a simple homogenous 11-residue sequence (alanine and glycine) with arginine and lysine at the $\mathrm{C}$ - and $\mathrm{N}$-terminal ends, respectively. While the central message of the study is the use of gas-phase HDX for structural interrogation, they do report that under fast HDX times (approximately 6 seconds) the sidechain and terminal amide groups are completely deuterated. Additionally, studies by Rand et al. confirm that side-chain deuteration may indicate elements of secondary structure as well as interactions associated with tertiary structure ${ }^{55}$. They were able to selectively identify charge sites in native ubiquitin through the combination of HDX and MS/MS analysis by ETD.

Many analytical techniques, such as electron microscopy (EM), atomic force microscopy (AFM), infrared spectroscopy (IR), fluorescence imaging, and $\mathrm{CD}$, only provide a global picture of protein aggregate morphology and secondary structure. Mass spectrometry (MS), however, is capable of site- 
specific analyses of intact proteins through the use of both "top-down" and "bottom-up" techniques coupled to isotopic labeling strategies, such as hydrogendeuterium exchange (HDX). ${ }^{57-59}$ "Bottom-up" HDX techniques are the most prevalent in the literature.$^{60-63}$ For these methods, protein labeling occurs in a deuterium-rich solvent, such as $\mathrm{D}_{2} \mathrm{O}$. The exchange is quenched by rapid addition of a highly protic solvent, such as formic or acetic acid, to $\mathrm{pH} 3$ and the temperature is decreased to $0^{\circ} \mathrm{C}$. The sample is then subjected to proteolysis, typically performed on-line, by an acid-active protease, such as pepsin. Following proteolysis, full MS and MS/MS analyses determine deuterium uptake and sites of deuterium exchange. This method has been used to study conformations of multiple proteins ${ }^{57,58,62}$ and aggregate species. ${ }^{64}$ However, a hallmark of amyloid proteins is a resistance to proteolysis due to the rapid formation of fibrils. Incomplete protein digestion presents problems in the forms of decreased peptide signal, column fouling and line clogging. Together these problems present a significant challenge in bottom-up HDX studies of aggregation-prone proteins.

Recent advancements in gas-phase fragmentation in MS/MS workflows allow for comparable sequence coverage through top-down fragmentation of intact proteins using electron transfer dissociation (ETD). ${ }^{65,66}$ Unlike collisioninduced dissociation (CID), which has been widely used in oxidative labeling studies, ${ }^{67,68}$ ETD preserves post-translational modifications ${ }^{69}$ and induces fewer deuterium scrambling events. ${ }^{58}$ To that end, top-down MS/MS workflows with ETD coupled to HDX and DHX have been routinely implemented in cases where 
the traditional bottom-up approach is not viable, or is more error-prone. Topdown HDX methods have been used for secondary structural characterization and structural transitions of intact dynamic biological systems on a millisecond timescale that is not possible with the more time-consuming traditional bottom-up technique. ${ }^{70,71}$ Additionally, the need for proteolytic digestion in bottom-up HDXMS studies can lead to loss of site-specific information due to back-exchange at the cleavage site. Since no proteolytic digestion is required, top-down techniques are also very well-suited for analysis of protein-ligand ${ }^{72}$ and soluble proteinprotein complexes. ${ }^{59}$ Early amyloid species are a specific subset of proteinprotein complex, and as such are amenable to top-down analyses.

Top-down studies have been performed for other amyloid proteins. ${ }^{59,73}$ In this approach, the protein is allowed to aggregate in a labeling environment such that nearly one hundred percent deuterium incorporation is achieved. The resulting aggregated structure is rapidly subjected to an "exchange-out" or hydrogen-rich buffer to remove all solvent-exposed, labile deuterium atoms (also called deuterium-hydrogen exchange, back-exchange, or DHX). Next, a highly protic organic buffer is used to quench the DHX reaction much like in the bottomup technique. The high organic content causes a rapid disaggregation and denaturing of the protein while leaving the label intact. Examination of exchange sites, both from the initial deuterium uptake and DHX, yield information about the structure of the protein and the protein complex. Very few studies have applied the top-down approach to amyloidogenic proteins and peptides; however, the bottom-up approach has been applied for amyloid $\beta,{ }^{74}$ and prion fibrils. ${ }^{75,76}$ The 
top-down approach has been used to characterize amyloid $\beta 1-40^{59}$ and $1-42^{73}$ oligomers. Pan et al. examine structural heterogeneity of amyloid $\beta$ and show deuterium uptake is a direct result of aggregate polymorphism. ${ }^{73}$ The top-down approach allows for selection of specific polymorphs due to differential deuterium uptake. This would not be possible with a bottom-up approach, because of the homogenization of peptide species from pepsin proteolysis.

\subsubsection{Covalent labeling for residue-specific structural interrogation.}

HDX and DHX can provide an adequate global representation of secondary protein structure, but it cannot completely confirm the presence of tertiary or quaternary interaction at a given residue. Covalent modification of a residue that was protected by deuterium exchange is a more direct, confirmatory test to determine involvement of a particular residue. Sites of covalent modification can be used to help determine the binding face in a given protein complex. Covalent modifications have been widely used to probe solvent accessibility of other amyloid proteins, such as $\beta 2$-microglobulin. ${ }^{67,68,77}$ These studies utilized diethylpyrocarbonate (DEPC) to carbethoxylate residues that have heteroatom-containing side chains. DEPC targets alcohol and amino groups, such as those found in $\mathrm{H}, \mathrm{T}, \mathrm{K}$, and $\mathrm{S}$.

Residue-specific label reactions and post-translational modifications can sometimes compromise the structural integrity of the native protein by altering hydrophobicity or introducing a new intramolecular interaction not present previously. ${ }^{78}$ Typically, sites of covalent modification are determined through a 
bottom-up approach; the protein is modified at increasing label concentrations, digested with a common enzyme, such as trypsin or chymotrypsin, and analyzed using reversed-phase liquid chromatography ${ }^{67,68,78,79}$ Modification can be reported as a normalized percent to the observed unmodified peptides. This percent modification can be compared in the context of complementary experiments, such as aggregation kinetics and/or morphology.

\subsection{Ion mobility-mass spectrometry}

\subsubsection{Ion mobility fundamentals.}

An ion mobility spectrometer is composed of an ionization source, a drift cell, and a detector. Once volatilized, the sample is ionized traditionally by ${ }^{63} \mathrm{Ni}$, a $\beta$ emitter ${ }^{80,81}$. A packet of ions containing the analyte enters the drift region through a shutter grid. The grid is electronically biased to collect ions until the potential is dropped. A potential gradient is applied to the drift region $( \pm 1-500 \mathrm{~V}$ $\mathrm{cm}^{-1}$ ) and ions move toward the detector. When the potential on the shutter grid is dropped, the ion packet is allowed into the drift region where the primary mode of separation is related to collisions with an inert drift gas (e.g., He). Analyte ions within the ion packet are separated according to their mobilities through the inert buffer gas. Fundamentally, the mobility of an ion $(K)$ is defined as the velocity of an ion through the drift tube $\left(v_{d}\right)$ divided by the electric field $(E)$ as shown from rearrangement of Equation 1.3.

$$
v_{d}=K E
$$


$v_{d}$ is related to ion drift time $\left(t_{D}\right)$ and drift tube length $(L)$ according to Equation 1.4:

$$
v_{d}=\frac{L}{t_{D}}
$$

Mobility is commonly reported in units of $\mathrm{cm}^{2} \mathrm{~V}^{-1} \mathrm{~s}^{-180,82}$. It is important to note that this definition of mobility refers to the average velocity for a population of the same ions. ${ }^{81}$. To allow for comparisons for measurements performed on different instruments, and/or those employing different experimental conditions, a reduced mobility $\left(\mathrm{K}_{0}\right)$ for the analyte ion is commonly reported. The reduced mobility is derived by normalizing to standard temperature and pressure as shown in Equation $1.5^{80,81}$.

$$
K_{0}=K\left(\frac{273.15}{T}\right)\left(\frac{P}{760}\right)
$$

Here, $\mathrm{T}$ is the buffer gas temperature, and $\mathrm{P}$ is the buffer gas pressure. Revercomb and Mason proposed what is now the most prevalent kinetic theory for ion separation in IMS ${ }^{82}$. They assume that a) ions are approximated by hard spheres and b) mobility depends upon momentum transfer resulting from collisions with the buffer gas. They arrive at the relationship shown in Equation 1.6 


$$
K=\frac{3}{16}\left(\frac{2 \pi}{\mu k_{b} T}\right)^{1 / 2}\left(\frac{z e}{N \Omega}\right)
$$

where $z$ is the charge of the ion, $e$ is the fundamental charge, $N$ is the number density of the drift gas, $\mu$ is the ion's reduced mass, $k_{B}$ is Boltzmann's constant, $T$ is the gas temperature, and $\Omega$ is the collisional cross-section of the ion ${ }^{82}$. It is instructive to note that mobility varies inversely with $\Omega$; that is, the ion mobility decreases with increasing size due to a proportional increase in collision frequency. Additionally, the mobility varies directly with charge (q) due to the relationship with the effective force exerted on the ion in the drift tube.

Rearrangement and expansion of Equation 6 for $\Omega$ yields Equation 1.7,

$$
\Omega=\frac{(18 \pi)^{1 / 2}}{16} \frac{z e}{\left(k_{b} T\right)^{1 / 2}}\left[\frac{1}{m_{I}}+\frac{1}{m_{B}}\right]^{1 / 2} \frac{t_{D} E}{L} \frac{760}{P} \frac{T}{273.2} \frac{1}{N}
$$

The IMS instrument used in these studies utilizes a dual-gating system. In IMStime of flight (ToF) MS, the ToF mass analysis is nested within the drift separation; ions are pushed into the ToF tube as they arrive from the mobility separation. ${ }^{83}$ In lieu of the ToF, the current work utilizes a linear ion trap (LIT) mass spectrometer. Since the LIT functions on a much slower time scale than the ToF, a dual-gating system is required where ions are introduced into the drift region by lowering the potential on a front electronic gate. ${ }^{84}$ lons are separated in the drift tube until they reach a second gate. The second gate is delayed relative to the first, such that only ions with drift times matching the gate delay are 
allowed into the mass spectrometer. ${ }^{85,86}$ Collecting a mass spectrum at each delay time creates a two-dimensional $t_{D}(\mathrm{~m} / \mathrm{z})$ spectrum. Ions can be concentrated in the LIT by focusing on a single drift time and optimizing trap parameters. This design also allows for multiple rounds of MS/MS to be performed on mobility-separated analytes, which is not possible with any other commercially available system. This instrument lends itself well to protein and peptide analyses because it provides structural selection and resolution with multi-stage tandem mass spectrometry.

\subsubsection{Ion mobility-mass spectrometry in bioanalysis and amyloid study.}

The structural heterogeneity of the htt system suggests the need for direct measurement of individual monomer and multimer species. High-resolution techniques, such as NMR, have been crucial to elucidating fibril structure; ${ }^{4,11,35}$, ${ }^{87}$ however, labeling multiple residues, as in the case for NMR, can become costprohibitive. Additionally, other spectroscopic methods, such as CD, only offer a global view of protein structure, as it cannot differentiate the structure of individual, coexisting protein or peptide conformers. Systems, such as Nt17, where structural heterogeneity could be a critical determinant in oligomer formation, require structurally selective methods to fully comprehend the interplay of secondary structure content and multimer formation. The work described in this document, in particular Chapters 3 and 4, utilize IMS-MS for structural characterization of peptide ions in the form of collision cross section determinations (see Equation 7, section 1.6.1). This cross section can be related 
to discrete secondary, tertiary, and quaternary structure through comparisons to in silico structures. ${ }^{88-95}$ If the protein is ionized at a low-energy, under native solution conditions, the gas-phase structures that are observed may be related to the solution structure. ${ }^{51,96,97}$ The observation of individual ion populations exhibiting different collision cross sections suggests the presence of multiple solution conformations. ${ }^{96,98}$ Collision cross sections can also track conformational abundances as a function of solution conditions. ${ }^{98,99}$

IMS-MS has seen widespread use in structural characterization of carbohydrates, peptides and proteins..$^{51,98,100-109}$ Traditional drift tube IMS (DTIMS), consisting of stacked ring electrodes providing a constant electric field $^{83,84,100}$ is used in the current study to examine native structural heterogeneity of a system whose oligomerization is predicated on formation of an amphipathic $\alpha$-helix (Nt17) ${ }^{12,}{ }^{26}$ The conformational resolution afforded by IMS is used to determine effects of covalent modification on secondary structure stability and multimer formation. Here, IMS-MS is used to directly monitor various monomer and multimer comformers.

IMS-MS has been used extensively in aggregate ${ }^{110}$ and early amyloid ${ }^{97,}$ $102,111-113$ characterization, primarily for the amyloid $\beta$ peptide $(A \beta)$. Specifically, IMS-MS revealed an oligomerization mechanism that occurred via multimer association for the $A \beta$ peptide. ${ }^{113}$ Annular hexameric structures could subsequently nucleate into dodecameric oligomers that are hypothesized as precursors for amyloid fibril formation, ${ }^{113}$ which demonstrates the ability of IMSMS to elucidate mechanistic details in aggregation phenomena. 


\subsubsection{Gas-phase deuterium exchange in IMS-MS.}

Condensed-phase deuterium exchange can provide residue-specific information regarding solvent accessibility and secondary structure; however, the reliability of these methods rely on the lack of back-exchange with aqueous environment, as well as operation under quenched conditions, which may not be suitable for some protein analytes. A second option is to perform the deuterium exchange experiment in the gas-phase. The gas-phase exchange depends largely on the protein analyte conformation and accessibility of a charge site, ${ }^{114}$ so structural information is retained. ${ }^{115}$ Much like the condensed phase experiments, proteins of varying conformation can still retain heterogeneous deuterium uptake patterns in the gas phase. ${ }^{86,116,117}$ One of the biggest advantages of gas-phase HDX is the lack of unwanted back-exchange to the bulk conditions, though deuterium scrambling by harsh MS environments is still a consideration.

Unlike solution phase exchange, which is catalyzed by the presence of OD`species and proceeds via a mechanism in which all available heteroatomic sites are labeled, ${ }^{118}$ gas-phase exchange proceeds by a relay mechanism in which the charge site and a less basic site of the peptide must be within proximity of one another. ${ }^{119}$ Additionally, heteroatomic sites that are buried within tertiary or quaternary arrangements such as salt bridges or other intermolecular interaction are not exchanged. The exchange begins with the establishment of a hydrogen bond between the charge site and an oxygen atom on $\mathrm{D}_{2} \mathrm{O}$. A second hydrogen bond is established between the less basic site on the peptide and the deuterium 
in the $\mathrm{D}_{2} \mathrm{O}$ molecule. The deuteron is transferred to the hydrophile, with concominant abstraction of a proton from the charge site. Subsequently, the deuteron may be abstracted by another basic site within the peptide. Thus, peptides with less accessible heteroatomic exchange sites are deuterated to a lesser extent than those with exposed heteroatom sites.

In some of the earliest work, three non-interconverting gas-phase cytochrome c conformers, that underwent deuterium exchange in the confines of a Fourier-transform ion cyclotron resonance MS (FTICR-MS), were observed, but not structurally resolved. ${ }^{117}$ Deuterium exchange after high-energy collisions then showed that at least six states are populated in the gas-phase, with deuterium uptake kinetics directly correlated with charge ${ }^{116}$ and, most importantly, lack of secondary structure. These studies showed that conformational heterogeneity is not only sampled in the gas-phase, but that by measuring the incorporation of deuterium atoms one could surmise the number of non-covalent interactions present in a given conformer. Since gas-phase deuterium exchange occurs on the order of $\mu \mathrm{s},{ }^{114-117,}{ }^{119}$ it can be performed simultaneously with IMS, in which residence time in the drift tube occurs on a ms time scale. Conformations of cytochrome c were simultaneously dispersed and deuterium-labeled in IMS, ${ }^{115}$ which demonstrated that compact conformations experienced fewer deuterium exchange events due to hydrogen protection. Deuteration levels observed in the FTICR experiment were much higher than those performed in the IMS separation, possibly because the FTICR was sensitive to longer time scale structural fluctuations. The benefit of gas-phase exchange in the IMS is the ability 
to structurally resolve each conformation, thus providing an extra layer of specificity.

The full utility of gas-phase deuterium exchange was not realized until recently; ${ }^{55,85,86}$ the advent of tandem mass spectrometry for mobility-resolved conformations via ETD allows for determination of deuterium incorporation sites without little consideration for deuterium scrambling. ${ }^{120}$ This technique can then be used to determine exact sites of hydrogen accessibility and uptake in gasphase structures and validate candidate structures generated by MDS ${ }^{86} \mathrm{~A}$ kinetic model was developed to relate deuterium uptake to charge site and carbonyl accessibility on model structures. ${ }^{121}$ Additionally, more recent experiments further detailed that charge site accessibility is required for increased deuterium exchange. Thus, in some cases, compact, lower charge conformers of a given peptide ion can have more deuterium exchange events than a higher-charge, more elongated conformation. ${ }^{86}$ Other studies have examined conformational rigidity with respect to disulfide bonds and Coulomb repulsion in deuterium exchange studies. ${ }^{122}$ In the current work, gas-phase deuterium exchange is used to probe accessibility of a heterodimeric complex and provide a model of intrinsic regulation of $\mathrm{Nt} 17$. 


\subsection{References}

(1) The Huntington's Disease Collaborative Research Group (1993) A NOVEL GENE CONTAINING A TRINUCLEOTIDE REPEAT THAT IS EXPANDED AND UNSTABLE ON HUNTINGTONS-DISEASE CHROMOSOMES, Cell 72, 971-983.

(2) Ross, C. A., and Tabrizi, S. J. (2011) Huntington's disease: from molecular pathogenesis to clinical treatment, Lancet Neurology 10, 83-98.

(3) DiFiglia, M., Sapp, E., Chase, K. O., Davies, S. W., Bates, G. P., Vonsattel, J. P., and Aronin, N. (1997) Aggregation of huntingtin in neuronal intranuclear inclusions and dystrophic neurites in brain, Science 277, 1990-1993.

(4) Hoop, C. L., Lin, H.-K., Kar, K., Hou, Z., Poirier, M. A., Wetzel, R., and van der Wel, P. C. A. (2014) Polyglutamine amyloid core boundaries and flanking domain dynamics in huntingtin fragment fibrils determined by solid-state NMR, Biochemistry.

(5) Bugg, C. W., Isas, J. M., Fischer, T., Patterson, P. H., and Langen, R. (2012) Structural Features and Domain Organization of Huntingtin Fibrils, Journal of Biological Chemistry 287, 31739-31746.

(6) Schneider, R., Schumacher, M. C., Mueller, H., Nand, D., Klaukien, V., Heise, H., Riedel, D., Wolf, G., Behrmann, E., Raunser, S., Seidel, R., Engelhard, M., and Baldus, M. (2011) Structural Characterization of Polyglutamine Fibrils by Solid-State NMR Spectroscopy, Journal of Molecular Biology 412, 121-136.

(7) Tobelmann, M. D., and Murphy, R. M. (2011) Location Trumps Length: Polyglutamine-Mediated Changes in Folding and Aggregation of a Host Protein, Biophysical Journal 100, 2773-2782.

(8) Arrasate, M., Mitra, S., Schweitzer, E. S., Segal, M. R., and Finkbeiner, S. (2004) Inclusion body formation reduces levels of mutant huntingtin and the risk of neuronal death, Nature 431, 805-810.

(9) Legleiter, J., Mitchell, E., Lotz, G. P., Sapp, E., Ng, C., DiFiglia, M., Thompson, L. M., and Muchowski, P. J. (2010) Mutant Huntingtin Fragments Form Oligomers in a Polyglutamine Length-dependent Manner in Vitro and in Vivo, Journal of Biological Chemistry 285, 14777-14790.

(10) Burke, K. A., Kauffman, K. J., Umbaugh, C. S., Frey, S. L., and Legleiter, J. (2013) The Interaction of Polyglutamine Peptides With Lipid Membranes is Regulated by Flanking Sequences Associated with Huntingtin, Journal of Biological Chemistry.

(11) Sivanandam, V. N., Jayaraman, M., Hoop, C. L., Kodali, R., Wetzel, R., and van der Wel, P. C. A. (2011) The Aggregation-Enhancing Huntingtin $\mathrm{N}$-Terminus Is Helical in Amyloid Fibrils, Journal of the American Chemical Society 133, 4558-4566.

(12) Jayaraman, M., Kodali, R., Sahoo, B., Thakur, A. K., Mayasundari, A., Mishra, R., Peterson, C. B., and Wetzel, R. (2012) Slow Amyloid Nucleation via alpha-Helix-Rich Oligomeric Intermediates in Short 
Polyglutamine-Containing Huntingtin Fragments, Journal of Molecular Biology 415, 881-899.

(13) Gouttenoire, J., Roingeard, P., Penin, F., and Moradpour, D. (2010) Amphipathic $\alpha$-helix $\mathrm{AH} 2$ is a major determinant for the oligomerization of hepatitis $\mathrm{C}$ virus nonstructural protein 4B, Journal of virology 84,12529 12537.

(14) Westerlund, J. A., and Weisgraber, K. H. (1993) Discrete carboxylterminal segments of apolipoprotein $\mathrm{E}$ mediate lipoprotein association and protein oligomerization, Journal of Biological Chemistry 268, 1574515750.

(15) Brass, V., Bieck, E., Montserret, R., Wölk, B., Hellings, J. A., Blum, H. E., Penin, F., and Moradpour, D. (2002) An Amino-terminal Amphipathic $\alpha-$ Helix Mediates Membrane Association of the Hepatitis C Virus Nonstructural Protein 5A, Journal of Biological Chemistry 277, 8130-8139.

(16) Georgieva, Elka R., Xiao, S., Borbat, Peter P., Freed, Jack H., and Eliezer, D. (2014) Tau Binds to Lipid Membrane Surfaces via Short Amphipathic Helices Located in Its Microtubule-Binding Repeats, Biophysical Journal 107, 1441-1452.

(17) Cui, H., Lyman, E., and Voth, G. A. (2011) Mechanism of Membrane Curvature Sensing by Amphipathic Helix Containing Proteins, Biophysical Journal 100, 1271-1279.

(18) Chaibva, M., Burke, K. A., and Legleiter, J. (2014) Curvature Enhances Binding and Aggregation of Huntingtin at Lipid Membranes, Biochemistry 53, 2355-2365.

(19) Nagarajan, A., Jawahery, S., and Matysiak, S. (2013) The Effects of Flanking Sequences in the Interaction of Polyglutamine Peptides with a Membrane Bilayer, The Journal of Physical Chemistry B.

(20) Kim, M. W., Chelliah, Y., Kim, S. W., Otwinowski, Z., and Bezprozvanny, I. (2009) Secondary Structure of Huntingtin Amino-Terminal Region, Structure 17, 1205-1212.

(21) Giulia Rossetti, P. C., Alessandro Laio, Paolo Carloni. (2011) Conformations of the Huntingtin $\mathrm{N}$-term in aqueous solution from atomistic simulations, FEBS Letters, 3086 - 3089.

(22) Dlugosz, M., and Trylska, J. (2011) Secondary Structures of Native and Pathogenic Huntingtin N-Terminal Fragments, Journal of Physical Chemistry B 115, 11597-11608.

(23) Thakur, A. K., Jayaraman, M., Mishra, R., Thakur, M., Chellgren, V. M., Byeon, I. J. L., Anjum, D. H., Kodali, R., Creamer, T. P., Conway, J. F., Gronenborn, A. M., and Wetzel, R. (2009) Polyglutamine disruption of the huntingtin exon $1 \mathrm{~N}$ terminus triggers a complex aggregation mechanism, Nature Structural \& Molecular Biology 16, 380-389.

(24) Kelley, N. W., Huang, X., Tam, S., Spiess, C., Frydman, J., and Pande, V. S. (2009) The Predicted Structure of the Headpiece of the Huntingtin Protein and Its Implications on Huntingtin Aggregation, Journal of Molecular Biology 388, 919-927. 
(25) Crick, S. L., Ruff, K. M., Garai, K., Frieden, C., and Pappu, R. V. (2013) Unmasking the roles of $\mathrm{N}$ - and $\mathrm{C}$-terminal flanking sequences from exon 1 of huntingtin as modulators of polyglutamine aggregation, Proceedings of the National Academy of Sciences 110, 20075-20080.

(26) Mishra, R., Jayaraman, M., Roland, B. P., Landrum, E., Fullam, T., Kodali, R., Thakur, A. K., Arduini, I., and Wetzel, R. (2012) Inhibiting the Nucleation of Amyloid Structure in a Huntingtin Fragment by Targeting alpha-Helix-Rich Oligomeric Intermediates, Journal of Molecular Biology 415, 900-917.

(27) Kelley, N. W., Huang, X. H., Tam, S., Spiess, C., Frydman, J., and Pande, V. S. (2009) The Predicted Structure of the Headpiece of the Huntingtin Protein and Its Implications on Huntingtin Aggregation, Journal of Molecular Biology 388, 919-927.

(28) Atwal, R. S., Xia, J., Pinchev, D., Taylor, J., Epand, R. M., and Truant, R. (2007) Huntingtin has a membrane association signal that can modulate huntingtin aggregation, nuclear entry and toxicity, Human Molecular Genetics 16, 2600-2615.

(29) Williamson, T. E., Vitalis, A., Crick, S. L., and Pappu, R. V. (2010) Modulation of Polyglutamine Conformations and Dimer Formation by the $\mathrm{N}$-Terminus of Huntingtin, Journal of Molecular Biology 396, 1295-1309.

(30) Vitalis, A., and Pappu, R. V. (2011) Assessing the contribution of heterogeneous distributions of oligomers to aggregation mechanisms of polyglutamine peptides, Biophysical Chemistry 159, 14-23.

(31) Nucifora, L. G., Burke, K. A., Feng, X., Arbez, N., Zhu, S. S., Miller, J., Yang, G. C., Ratovitski, T., Delannoy, M., Muchowski, P. J., Finkbeiner, S., Legleiter, J., Ross, C. A., and Poirier, M. A. (2012) Identification of Novel Potentially Toxic Oligomers Formed in Vitro from Mammalianderived Expanded huntingtin Exon-1 Protein, Journal of Biological Chemistry 287, 16017-16028.

(32) Olshina, M. A., Angley, L. M., Ramdzan, Y. M., Tang, J. W., Bailey, M. F., Hill, A. F., and Hatters, D. M. (2010) Tracking Mutant Huntingtin Aggregation Kinetics in Cells Reveals Three Major Populations That Include an Invariant Oligomer Pool, Journal of Biological Chemistry 285, 21807-21816.

(33) Jayaraman, M., Mishra, R., Kodali, R., Thakur, A. K., Koharudin, L. M. I., Gronenborn, A. M., and Wetzel, R. (2012) Kinetically Competing Huntingtin Aggregation Pathways Control Amyloid Polymorphism and Properties, Biochemistry 51, 2706-2716.

(34) Burke, K. A., Hensal, K. M., Umbaugh, C. S., Chaibva, M., and Legleiter, J. (2013) Huntingtin disrupts lipid bilayers in a polyQ-length dependent manner, Biochimica et Biophysica Acta (BBA) - Biomembranes 1828, 1953-1961.

(35) Kar, K., Hoop, C. L., Drombosky, K. W., Baker, M. A., Kodali, R., Arduini, I., van der Wel, P. C. A., Horne, W. S., and Wetzelt, R. (2013) betaHairpin-Mediated Nucleation of Polyglutamine Amyloid Formation, Journal of Molecular Biology 425, 1183-1197. 
(36) Caron, N. S., Desmond, C. R., Xia, J., and Truant, R. (2013) Polyglutamine domain flexibility mediates the proximity between flanking sequences in huntingtin, Proceedings of the National Academy of Sciences 110, 14610-14615.

(37) Kar, K., Jayaraman, M., Sahoo, B., Kodali, R., and Wetzel, R. (2011) Critical nucleus size for disease-related polyglutamine aggregation is repeat-length dependent, Nature Structural \& Molecular Biology 18, 328-+.

(38) Tam, S., Spiess, C., Auyeung, W., Joachimiak, L., Chen, B., Poirier, M. A., and Frydman, J. (2009) The chaperonin TRiC blocks a huntingtin sequence element that promotes the conformational switch to aggregation, Nature Structural \& Molecular Biology 16, 1279-U1298.

(39) Tam, S., Geller, R., Spiess, C., and Frydman, J. (2006) The chaperonin TRiC controls polyglutamine aggregation and toxicity through subunitspecific interactions, Nature cell biology 8, 1155-1162.

(40) Lotz, G. P., Legleiter, J., Aron, R., Mitchell, E. J., Huang, S. Y., Ng, C. P., Glabe, C., Thompson, L. M., and Muchowski, P. J. (2010) Hsp70 and Hsp40 Functionally Interact with Soluble Mutant Huntingtin Oligomers in a Classic ATP-dependent Reaction Cycle, Journal of Biological Chemistry 285, 38183-38193.

(41) Legleiter, J., Lotz, G. P., Miller, J., Ko, J., Ng, C., Williams, G. L., Finkbeiner, S., Patterson, P. H., and Muchowski, P. J. (2009) Monoclonal Antibodies Recognize Distinct Conformational Epitopes Formed by Polyglutamine in a Mutant Huntingtin Fragment, Journal of Biological Chemistry 284, 21647-21658.

(42) Khoshnan, A., Ko, J., and Patterson, P. H. (2002) Effects of intracellular expression of anti-huntingtin antibodies of various specificities on mutant huntingtin aggregation and toxicity, Proceedings of the National Academy of Sciences 99, 1002-1007.

(43) Peters-Libeu, C., Newhouse, Y., Krishnan, P., Cheung, K., Brooks, E., Weisgraber, K., and Finkbeiner, S. (2005) Crystallization and diffraction properties of the Fab fragment of 3B5H10, an antibody specific for disease-causing polyglutamine stretches, Acta Crystallographica Section F: Structural Biology and Crystallization Communications 61, 1065-1068.

(44) Colby, D. W., Garg, P., Holden, T., Chao, G., Webster, J. M., Messer, A., Ingram, V. M., and Wittrup, K. D. (2004) Development of a Human Light Chain Variable Domain (VL) Intracellular Antibody Specific for the Amino Terminus of Huntingtin via Yeast Surface Display, Journal of Molecular Biology 342, 901-912.

(45) Colby, D. W., Chu, Y., Cassady, J. P., Duennwald, M., Zazulak, H., Webster, J. M., Messer, A., Lindquist, S., Ingram, V. M., and Wittrup, K. D. (2004) Potent inhibition of huntingtin aggregation and cytotoxicity by a disulfide bond-free single-domain intracellular antibody, Proceedings of the National Academy of Sciences of the United States of America 101, 17616-17621.

(46) Lecerf, J. M., Shirley, T. L., Zhu, Q., Kazantsev, A., Amersdorfer, P., Housman, D. E., Messer, A., and Huston, J. S. (2001) Human single-chain 
Fv intrabodies counteract in situ huntingtin aggregation in cellular models of Huntington's disease, Proceedings of the National Academy of Sciences of the United States of America 98, 4764-4769.

(47) Fenn, J., Mann, M., Meng, C., Wong, S., and Whitehouse, C. (1989) Electrospray ionization for mass spectrometry of large biomolecules, Science 246, 64-71.

(48) Sterling, H. J., Cassou, C. A., Susa, A. C., and Williams, E. R. (2012) Electrothermal Supercharging of Proteins in Native Electrospray Ionization, Analytical Chemistry 84, 3795-3801.

(49) Fernandez de la Mora, J. (2000) Electrospray ionization of large multiply charged species proceeds via Dole's charged residue mechanism, Analytica Chimica Acta 406, 93-104.

(50) Enke, C. G. (1997) A Predictive Model for Matrix and Analyte Effects in Electrospray Ionization of Singly-Charged Ionic Analytes, Analytical Chemistry 69, 4885-4893.

(51) Wyttenbach, T., and Bowers, M. T. (2011) Structural Stability from Solution to the Gas Phase: Native Solution Structure of Ubiquitin Survives Analysis in a Solvent-Free Ion Mobility-Mass Spectrometry Environment, Journal of Physical Chemistry B 115, 12266-12275.

(52) Cassou, C. A., Sterling, H. J., Susa, A. C., and Williams, E. R. (2012) Electrothermal Supercharging in Mass Spectrometry and Tandem Mass Spectrometry of Native Proteins, Analytical Chemistry 85, 138-146.

(53) Smith, D. L., Deng, Y. Z., and Zhang, Z. Q. (1997) Probing the noncovalent structure of proteins by amide hydrogen exchange and mass spectrometry, Journal of Mass Spectrometry 32, 135-146.

(54) Coon, J. J., Ueberheide, B., Syka, J. E. P., Dryhurst, D. D., Ausio, J., Shabanowitz, J., and Hunt, D. F. (2005) Protein identification using sequential ion/ion reactions and tandem mass spectrometry, Proceedings of the National Academy of Sciences of the United States of America 102, 9463-9468.

(55) Rand, K. D., Pringle, S. D., Morris, M., and Brown, J. M. (2012) SiteSpecific Analysis of Gas-Phase Hydrogen/Deuterium Exchange of Peptides and Proteins by Electron Transfer Dissociation, Analytical Chemistry 84, 1931-1940.

(56) Pan, J., Heath, B. L., Jockusch, R. A., and Konermann, L. (2011) Structural Interrogation of Electrosprayed Peptide lons by Gas-Phase H/D Exchange and Electron Capture Dissociation Mass Spectrometry, Analytical Chemistry 84, 373-378.

(57) Engen, J. R., Wales, T. E., Chen, S. G., Marzluff, E. M., Hassell, K. M., Weis, D. D., and Smithgall, T. E. (2013) Partial cooperative unfolding in proteins as observed by hydrogen exchange mass spectrometry, International Reviews in Physical Chemistry 32, 96-127.

(58) Landgraf, R. R., Chalmers, M. J., and Griffin, P. R. (2012) Automated Hydrogen/Deuterium Exchange Electron Transfer Dissociation High Resolution Mass Spectrometry Measured at Single-Amide Resolution, Journal of the American Society for Mass Spectrometry 23, 301-309. 
(59) Pan, J. X., Han, J., Borchers, C. H., and Konermann, L. (2012) Structure and Dynamics of Small Soluble A beta(1-40) Oligomers Studied by TopDown Hydrogen Exchange Mass Spectrometry, Biochemistry 51, 36943703.

(60) Burns, K. M., Rey, M., Baker, C. A. H., and Schriemer, D. C. (2013) Platform Dependencies in Bottom-up Hydrogen/Deuterium Exchange Mass Spectrometry, Molecular \& Cellular Proteomics 12, 539-548.

(61) Percy, A. J., Slysz, G. W., and Schriemer, D. C. (2009) Surrogate H/D Detection Strategy for Protein Conformational Analysis Using MS/MS Data, Analytical Chemistry 81, 7900-7907.

(62) Ahn, J., Jung, M. C., Wyndham, K., Yu, Y. Q., and Engen, J. R. (2012) Pepsin Immobilized on High-Strength Hybrid Particles for Continuous Flow Online Digestion at 10000 psi, Analytical Chemistry 84, 7256-7262.

(63) Morgan, C. R., Hebling, C. M., Rand, K. D., Stafford, D. W., Jorgenson, J. W., and Engen, J. R. (2011) Conformational Transitions in the Membrane Scaffold Protein of Phospholipid Bilayer Nanodiscs, Molecular \& Cellular Proteomics 10.

(64) Huang, R. Y. C., Garai, K., Frieden, C., and Gross, M. L. (2011) Hydrogen/Deuterium Exchange and Electron-Transfer Dissociation Mass Spectrometry Determine the Interface and Dynamics of Apolipoprotein $E$ Oligomerization, Biochemistry 50, 9273-9282.

(65) Rozman, M., and Gaskell, S. J. (2012) Charge state dependent top-down characterisation using electron transfer dissociation, Rapid Communications in Mass Spectrometry 26, 282-286.

(66) Drabik, A., Bodzon-Kulakowska, A., and Suder, P. (2012) Application of the ETD/PTR reactions in top-down proteomics as a faster alternative to bottom-up nanoLC-MS/MS protein identification, Journal of Mass Spectrometry 47, 1347-1352.

(67) Mendoza, V. L., Baron-Rodriguez, M. A., Blanco, C., and Vachet, R. W. (2011) Structural Insights into the Pre-Amyloid Tetramer of beta-2Microglobulin from Covalent Labeling and Mass Spectrometry, Biochemistry 50, 6711-6722.

(68) Mendoza, V. L., Antwi, K., Baron-Rodriguez, M. A., Blanco, C., and Vachet, R. W. (2010) Structure of the Preamyloid Dimer of beta-2Microglobulin from Covalent Labeling and Mass Spectrometry, Biochemistry 49, 1522-1532.

(69) Tsybin, Y. O., Fornelli, L., Stoermer, C., Luebeck, M., Parra, J., Nallet, S., Wurm, F. M., and Hartmer, R. (2011) Structural Analysis of Intact Monoclonal Antibodies by Electron Transfer Dissociation Mass Spectrometry, Analytical Chemistry 83, 8919-8927.

(70) Pan, J., Han, J., Borchers, C. H., and Konermann, L. (2010) Characterizing Short-Lived Protein Folding Intermediates by Top-Down Hydrogen Exchange Mass Spectrometry, Analytical Chemistry 82, 85918597.

(71) Pan, J., Han, J., Borchers, C. H., and Konermann, L. (2009) Hydrogen/Deuterium Exchange Mass Spectrometry with Top-Down

1. Introduction: Insights to neurodegenerative disease provided by deuterium exchange and ion mobility-mass spectrometry 
Electron Capture Dissociation for Characterizing Structural Transitions of a $17 \mathrm{kDa}$ Protein, Journal of the American Chemical Society 131, 1280112808.

(72) Hopper, E. D., Roulhac, P. L., Campa, M. J., Patz, E. F., Jr., and Fitzgerald, M. C. (2008) Throughput and efficiency of a mass spectrometry-based screening assay for protein-ligand binding detection, J Am Soc Mass Spectrom 19, 1303-1311.

(73) Pan, J., Han, J., Borchers, C. H., and Konermann, L. (2011) ConformerSpecific Hydrogen Exchange Analysis of $A \beta(1-42)$ Oligomers by TopDown Electron Capture Dissociation Mass Spectrometry, Analytical Chemistry 83, 5386-5393.

(74) Kodali, R., Williams, A. D., Chemuru, S., and Wetzel, R. (2010) A $\beta(1-40)$ Forms Five Distinct Amyloid Structures whose $\beta$-Sheet Contents and Fibril Stabilities Are Correlated, Journal of Molecular Biology 401, 503-517.

(75) Lu, X., Wintrode, P. L., and Surewicz, W. K. (2007) $\beta$-Sheet core of human prion protein amyloid fibrils as determined by hydrogen/deuterium exchange, Proceedings of the National Academy of Sciences 104, 1510 1515.

(76) Nazabal, A., Dos Reis, S., Bonneu, M., Saupe, S. J., and Schmitter, J.-M. (2003) Conformational Transition Occurring upon Amyloid Aggregation of the HET-s Prion Protein of Podospora anserina Analyzed by Hydrogen/Deuterium Exchange and Mass Spectrometry†, Biochemistry 42, 8852-8861.

(77) Stocks, B. B., Rezvanpour, A., Shaw, G. S., and Konermann, L. (2011) Temporal Development of Protein Structure during S100A11 Folding and Dimerization Probed by Oxidative Labeling and Mass Spectrometry, Journal of Molecular Biology 409, 669-679.

(78) Mendoza, V. L., and Vachet, R. W. (2009) Probing protein structure by amino acid-specific covalent labeling and mass spectrometry, Mass Spectrometry Reviews 28, 785-815.

(79) Mendoza, V. L., and Vachet, R. W. (2008) Protein Surface Mapping Using Diethylpyrocarbonate with Mass Spectrometric Detection, Analytical Chemistry 80, 2895-2904.

(80) Creaser, C. S., Griffiths, J. R., Bramwell, C. J., Noreen, S., Hill, C. A., and Thomas, C. L. P. (2004) Ion mobility spectrometry: a review. Part 1. Structural analysis by mobility measurement, Analyst 129, 984-994.

(81) Eiceman, G. A. K. Z. (2005) Ion Mobility Spectrometry, 2nd ed., CRC Press, Boca Raton, FL.

(82) Revercomb, H. E., and Mason, E. A. (1975) THEORY OF PLASMA CHROMATOGRAPHY GASEOUS ELECTROPHORESIS - REVIEW, Analytical Chemistry 47, 970-983.

(83) Hoaglund, C. S., Valentine, S. J., Sporleder, C. R., Reilly, J. P., and Clemmer, D. E. (1998) Three-Dimensional Ion Mobility/TOFMS Analysis of Electrosprayed Biomolecules, Analytical Chemistry 70, 2236-2242. 
(84) Wu, C., Siems, W. F., Asbury, G. R., and Hill, H. H. (1998) Electrospray Ionization High-Resolution Ion Mobility Spectrometry-Mass Spectrometry, Analytical Chemistry 70, 4929-4938.

(85) Donohoe, G. C., Maleki, H., Arndt, J. R., Khakinejad, M., Yi, J., McBride, C., Nurkiewicz, T. R., and Valentine, S. J. (2014) A New lon MobilityLinear Ion Trap Instrument for Complex Mixture Analysis, Analytical Chemistry 86, 8121-8128.

(86) Khakinejad, M., Kondalaji, S. G., Maleki, H., Arndt, J. R., Donohoe, G. C., and Valentine, S. J. (2014) Combining lon Mobility Spectrometry with Hydrogen-Deuterium Exchange and Top-Down MS for Peptide Ion Structure Analysis, Journal of The American Society for Mass Spectrometry 25, 2103-2115.

(87) Michalek, M., Salnikov, E. S., Werten, S., and Bechinger, B. (2013) Membrane Interactions of the Amphipathic Amino Terminus of Huntingtin, Biochemistry 52, 847-858.

(88) Wyttenbach, T., von Helden, G., and Bowers, M. T. (1996) Gas-Phase Conformation of Biological Molecules: Bradykinin, Journal of the American Chemical Society 118, 8355-8364.

(89) Counterman, A. E., and Clemmer, D. E. (2002) Cis-Trans Signatures of Proline-Containing Tryptic Peptides in the Gas Phase, Analytical Chemistry 74, 1946-1951.

(90) Taraszka, J. A., Counterman, A. E., and Clemmer, D. E. (2001) Large anhydrous polyalanine ions: substitution of $\mathrm{Na}+$ for $\mathrm{H}+$ destabilizes folded states, International Journal of Mass Spectrometry 204, 87-100.

(91) Mao, Y., Woenckhaus, J., Kolafa, J., Ratner, M. A., and Jarrold, M. F. (1999) Thermal Unfolding of Unsolvated Cytochrome c: Experiment and Molecular Dynamics Simulations, Journal of the American Chemical Society 121, 2712-2721.

(92) Hall, Z., Politis, A., and Robinson, C. V. (2012) Structural modeling of heteromeric protein complexes from disassembly pathways and ion mobility-mass spectrometry, Structure 20, 1596-1609.

(93) Politis, A., Park, A. Y., Hyung, S.-J., Barsky, D., Ruotolo, B. T., and Robinson, C. V. (2010) Integrating Ion Mobility Mass Spectrometry with Molecular Modelling to Determine the Architecture of Multiprotein Complexes, PLoS ONE 5, e12080.

(94) Ruotolo, B. T., Hyung, S.-J., Robinson, P. M., Giles, K., Bateman, R. H., and Robinson, C. V. (2007) Ion Mobility-Mass Spectrometry Reveals Long-Lived, Unfolded Intermediates in the Dissociation of Protein Complexes, Angewandte Chemie International Edition 46, 8001-8004.

(95) Gidden, J., Kemper, P. R., Shammel, E., Fee, D. P., Anderson, S., and Bowers, M. T. (2003) Application of ion mobility to the gas-phase conformational analysis of polyhedral oligomeric silsesquioxanes (POSS), International Journal of Mass Spectrometry 222, 63-73.

(96) Pierson, N. A., Chen, L., Valentine, S. J., Russell, D. H., and Clemmer, D. E. (2011) Number of Solution States of Bradykinin from Ion Mobility and 
Mass Spectrometry Measurements, Journal of the American Chemical Society 133, 13810-13813.

(97) Bernstein, S. L., Dupuis, N. F., Lazo, N. D., Wyttenbach, T., Condron, M. M., Bitan, G., Teplow, D. B., Shea, J. E., Ruotolo, B. T., Robinson, C. V., and Bowers, M. T. (2009) Amyloid-beta protein oligomerization and the importance of tetramers and dodecamers in the aetiology of Alzheimer's disease, Nature Chemistry 1, 326-331.

(98) Shi, H. L., Pierson, N. A., Valentine, S. J., and Clemmer, D. E. (2012) Conformation Types of Ubiquitin $\mathrm{M}+8 \mathrm{H}(8+)$ lons from Water:Methanol Solutions: Evidence for the $\mathrm{N}$ and $\mathrm{A}$ States in Aqueous Solution, Journal of Physical Chemistry B 116, 3344-3352.

(99) Shi, L., Holliday, A. E., Shi, H., Zhu, F., Ewing, M. A., Russell, D. H., and Clemmer, D. E. (2014) Characterizing Intermediates Along the Transition from Polyproline I to Polyproline II Using Ion Mobility Spectrometry-Mass Spectrometry, Journal of the American Chemical Society.

(100) Hudgins, R. R., Woenckhaus, J., and Jarrold, M. F. (1997) High resolution ion mobility measurements for gas phase proteins: correlation between solution phase and gas phase conformations, International Journal of Mass Spectrometry and lon Processes 165-166, 497-507.

(101) Clemmer, D. E., Hudgins, R. R., and Jarrold, M. F. (1995) Naked Protein Conformations: Cytochrome $\mathrm{c}$ in the Gas Phase, Journal of the American Chemical Society 117, 10141-10142.

(102) Bleiholder, C., Do, T. D., Wu, C., Economou, N. J., Bernstein, S. S., Buratto, S. K., Shea, J. E., and Bowers, M. T. (2013) Ion Mobility Spectrometry Reveals the Mechanism of Amyloid Formation of A beta(2535) and Its Modulation by Inhibitors at the Molecular Level:

Epigallocatechin Gallate and Scyllo-inositol, Journal of the American Chemical Society 135, 16926-16937.

(103) Li, H., Bendiak, B., Siems, W. F., Gang, D. R., and Hill, H. H. (2013) Carbohydrate Structure Characterization by Tandem Ion Mobility Mass Spectrometry (IMMS)2, Analytical Chemistry 85, 2760-2769.

(104) Kloniecki, M., Jablonowska, A., Poznanski, J., Langridge, J., Hughes, C., Campuzano, I., Giles, K., and Dadlez, M. (2011) Ion Mobility Separation Coupled with MS Detects Two Structural States of Alzheimer's Disease A beta 1-40 Peptide Oligomers, Journal of Molecular Biology 407, 110-124.

(105) Bornschein, R. E., Hyung, S. J., and Ruotolo, B. T. (2011) lon MobilityMass Spectrometry Reveals Conformational Changes in Charge Reduced Multiprotein Complexes, Journal of the American Society for Mass Spectrometry 22, 1690-1698.

(106) Trimpin, S., Tan, B., Bohrer, B. C., O'Dell, D. K., Merenbloom, S. I., Pazos, M. X., Clemmer, D. E., and Walker, J. M. (2009) Profiling of phospholipids and related lipid structures using multidimensional ion mobility spectrometry-mass spectrometry, International Journal of Mass Spectrometry 287, 58-69.

(107) Shvartsburg, A. A., Li, F. M., Tang, K. Q., and Smith, R. D. (2006) Characterizing the structures and folding of free proteins using 2-D gas-

1. Introduction: Insights to neurodegenerative disease provided by deuterium exchange and ion mobility-mass spectrometry 
phase separations: Observation of multiple unfolded conformers, Analytical Chemistry 78, 3304-3315.

(108) Ridenour, W. B., Kliman, M., McLean, J. A., and Caprioli, R. M. (2010) Structural Characterization of Phospholipids and Peptides Directly from Tissue Sections by MALDI Traveling-Wave Ion Mobility-Mass Spectrometry, Analytical Chemistry 82, 1881-1889.

(109) Campuzano, I., Bush, M. F., Robinson, C. V., Beaumont, C., Richardson, K., Kim, H., and Kim, H. I. (2011) Structural Characterization of Drug-like Compounds by lon Mobility Mass Spectrometry: Comparison of Theoretical and Experimentally Derived Nitrogen Collision Cross Sections, Analytical Chemistry 84, 1026-1033.

(110) Counterman, A. E., Valentine, S. J., Srebalus, C. A., Henderson, S. C., Hoaglund, C. S., and Clemmer, D. E. (1998) High-order structure and dissociation of gaseous peptide aggregates that are hidden in mass spectra, Journal of the American Society for Mass Spectrometry 9, 743759.

(111) Gessel, M. M., Wu, C., Li, H. Y., Bitan, G., Shea, J. E., and Bowers, M. T. (2012) A beta(39-42) Modulates A beta Oligomerization but Not Fibril Formation, Biochemistry 51, 108-117.

(112) Bleiholder, C., Dupuis, N. F., Wyttenbach, T., and Bowers, M. T. (2011) Ion mobility-mass spectrometry reveals a conformational conversion from random assembly to beta-sheet in amyloid fibril formation, Nature Chemistry 3, 172-177.

(113) Bernstein, S. L., Wyttenbach, T., Baumketner, A., Shea, J. E., Bitan, G., Teplow, D. B., and Bowers, M. T. (2005) Amyloid beta-protein: Monomer structure and early aggregation states of A beta 42 and its Pro(19) alloform, Journal of the American Chemical Society 127, 2075-2084.

(114) Campbell, S., Rodgers, M. T., Marzluff, E. M., and Beauchamp, J. L. (1995) Deuterium exchange reactions as a probe of biomolecule structure. Fundamental studies of cas phase $\mathrm{H} / \mathrm{D}$ exchange reactions of protonated glycine oligomers with D2O, CD3OD, CD3CO2D, and ND3, Journal of the American Chemical Society 117, 12840-12854.

(115) Valentine, S. J., and Clemmer, D. E. (1997) H/D exchange levels of shape-resolved cytochrome c conformers in the gas phase, Journal of the American Chemical Society 119, 3558-3566.

(116) Wood, T. D., Chorush, R. A., Wampler, F. M., Little, D. P., Oconnor, P. B., and McLafferty, F. W. (1995) GAS-PHASE FOLDING AND UNFOLDING OF CYTOCHROME-C CATIONS, Proceedings of the National Academy of Sciences of the United States of America 92, 2451-2454.

(117) Suckau, D., Shi, Y., Beu, S. C., Senko, M. W., Quinn, J. P., Wampler, F. M., and McLafferty, F. W. (1993) COEXISTING STABLE CONFORMATIONS OF GASEOUS PROTEIN IONS, Proceedings of the National Academy of Sciences of the United States of America 90, 790793. 
(118) Wales, T. E., and Engen, J. R. (2006) Hydrogen exchange mass spectrometry for the analysis of protein dynamics, Mass Spectrometry Reviews 25, 158-170.

(119) Wyttenbach, T., and Bowers, M. T. (1999) Gas phase conformations of biological molecules: the hydrogen/deuterium exchange mechanism, Journal of the American Society for Mass Spectrometry 10, 9-14.

(120) Abzalimov, R. R., Kaplan, D. A., Easterling, M. L., and Kaltashov, I. A. (2009) Protein Conformations Can Be Probed in Top-Down HDX MS Experiments Utilizing Electron Transfer Dissociation of Protein lons Without Hydrogen Scrambling, Journal of the American Society for Mass Spectrometry 20, 1514-1517.

(121) Khakinejad, M. K., S; Valentine, S. (2015) Gas-phase hydrogen-deuterium exchange labeling of select peptide ion conformer types: a per-residue kinetics analysis, Journal of The American Society for Mass Spectrometry In Press.

(122) Donohoe, G., Khakinejad, M., and Valentine, S. (2015) Ion Mobility Spectrometry-Hydrogen Deuterium Exchange Mass Spectrometry of Anions: Part 1. Peptides to Proteins, Journal of The American Society for Mass Spectrometry 26, 564-576. 


\section{Lysine Residues in the N-Terminal Huntingtin Amphipathic $\alpha$-Helix Play a Key Role in Peptide Aggregation ${ }^{1}$}

${ }^{1}$ Reproduced with permission from the Journal of Mass Spectrometry: Lysine residues in the Nterminal huntingtin amphipathic $\alpha$-helix play a key role in peptide aggregation. Arndt, J. R., Brown, R. J., Burke, K. A., Legleiter, J. and Valentine, S. J. Journal of Mass Spectrometry 50, 117-126. Copyright (C) 2015 John Wiley \& Sons, Ltd.

Huntington's Disease (HD) is a genetic neurodegenerative disorder caused by an expansion in a polyglutamine domain near the $\mathrm{N}$-terminus of the huntingtin (htt) protein that results in the formation of protein aggregates. The focus of the study is on the 17 residue $\mathrm{N}$-terminal flanking region of the peptide (Nt17) that has been shown to alter htt aggregation kinetics and morphology. A top-down sequencing strategy employing electron transfer dissociation (ETD) is utilized to determine the location of accessible and protected hydrogens in the aggregate. Evidence for protected residues is observed in the Nt17 tract and specifically point to lysine residues as potentially playing a significant role in $\mathrm{htt}$ aggregation.

\subsection{Introduction: The case for deuterium exchange MS in HD characterization}

Huntington's disease (HD) is a fatal neurodegenerative disease caused by an expanded CAG repeat in the huntingtin gene that codes for a polyglutamine (polyQ) region in exon 1 of the huntingtin (htt) protein. ${ }^{1}$ Neurotoxicity is thought to derive from the aggregation of the polyQ tract and has been shown to be repeat-length dependent with an expansion beyond $\sim 35 \mathrm{Q}$ representing the threshold for initiating $\mathrm{HD} \cdot{ }^{1-3}$ Although expansion of the polyQ region drives aggregation, the seventeen-residue $\mathrm{N}$-terminal 
flanking sequence (Nt17) enhances aggregation rates by promoting the formation of an oligomeric intermediate to fibril formation in vitro, ${ }^{4,5}$ and altering $\mathrm{Nt} 17$ is an effective strategy to modify the aggregation process. ${ }^{6} \mathrm{Nt} 17$ has been shown to contain $\alpha$-helical content by a variety of techniques, including circular dichroism (CD), ${ }^{4,7}$ nuclear magnetic resonance (NMR),${ }^{8}$ molecular dynamic simulations, ${ }^{9}$ and $x$-ray crystallography. ${ }^{10}$ This a-helix is amphipathic in nature, driving htt's interaction with lipid membranes. ${ }^{5,11-15}$ This amphiphilic, helical nature of Nt17 appears to underlie its ability to enhance fibril formation, nuclear accumulation, and toxicity. ${ }^{716}$ As a result, considerable effort has been aimed at elucidating the fate of the Nt17 domain in the various aggregate forms of htt. ${ }^{8,17,18}$

Huntingtin aggregation is thought to occur through a complex mechanism with several possible structural intermediates. ${ }^{4,19}$ A tetrameric species, whose formation is driven by Nt17, may represent the critical nucleus for aggregation. ${ }^{4}$ Inter-helical interaction associated with $\mathrm{Nt} 17$ is believed to be primarily hydrophobic in nature. ${ }^{16,20}$ Furthermore, mutations or modifications in the Nt17 domain slow aggregation, specifically serine phosphorylation or serine mutation to arginine ${ }^{21-23}$ Curiously, these modifications fall in the hydrophilic region of the helix, suggesting that more than the hydrophobic interaction drives inter-helical association. Elucidating specific interactions in the formation of htt aggregates is a primary focus within the field ${ }^{8,11,16,24}$ with far reaching implications in devising therapeutic strategies. ${ }^{6,23}$ The arguments presented above demonstrate that adequate structural characterization of htt aggregate species requires a residue-specific analysis. HD-related peptides have traditionally been studied by spectroscopic and microscopic techniques. $8,13,17,19,25-28$ 
The purpose of this study is to extend these top-down proteomics techniques for the elucidation of the three-dimensional arrangement of an aggregation-enhancing tract in a truncated htt peptide. ${ }^{8}$ This truncated peptide contains the Nt17 domain, a polyglutamine domain 35 residues in length (representative of disease threshold), a 10 residue polyproline domain, and two lysine residues on the C-terminus side of the polyQ domain to aid in solubility. Here, this peptide will be referred to as $N t 17 Q_{35} P_{10} K_{2}$. The aggregates formed from this synthetic model do not significantly differ from those formed from htt exon 1 cellular constructs. The C-terminal lysine residues do slightly increase aggregation kinetics relative to a full htt exon 1 construct; however, morphologically, aggregates are morphologically indistinguishable at identical time points. ${ }^{29}$ Thus, the C-terminal lysine residues have little bearing on aggregate kinetics or morphology. ${ }^{29}$ This represents the first time top-down mass spectrometry techniques have been applied for structural characterization of htt aggregates.

\subsection{Materials and Methods}

\subsubsection{Sample Preparation.}

Synthetic $\mathrm{Nt} 17 \mathrm{Q}_{35} \mathrm{P}_{10} \mathrm{~K}_{2}$ was purchased from the Keck institute (Yale University) and stored at $-20^{\circ} \mathrm{C}$ until later use. Disaggregation and solubilization of peptides was achieved by a variation on published protocols. ${ }^{30} \mathrm{In}$ short, $4.0 \mathrm{mg}$ portions were dissolved in $1.00 \mathrm{~mL}$ of 50/50 trifluoroacetic acid/hexafluoroisopropanol (TFA/HFIP) to ensure disaggregation. The samples were aliquoted into $250 \mu \mathrm{L}$ portions and dried under a steady stream of nitrogen until most of the TFA/HFIP was removed. The aliquots were then placed in a centrifugal evaporator for two hours to ensure removal of 
all TFA/HFIP. The resulting $1.0 \mathrm{mg}$ peptide films were stored at $-20^{\circ} \mathrm{C}$ until further use. The peptide films were reconstituted in $20 \mu \mathrm{L}$ of pH 3 TFA and diluted ten-fold into $\mathrm{D}_{2} \mathrm{O}$ with $100 \mathrm{mM}$ ammonium acetate (labeling buffer, $\mathrm{pH} 7.0$ ) to obtain a final concentration of $\sim 5.0 \mathrm{mg} \cdot \mathrm{mL}^{-1}$. This peptide concentration, although above the normal physiological level, compensates for the loss in signal due to dilution into the DHX buffer. Typical protocols call for the use of phosphate or tris buffers for incubation; however, those are not suitable for mass spectrometry. For this reason, ammonium acetate was chosen, as it is a much more amenable salt for mass spectrometry, as a pH stabilizer. Samples were allowed to rest in the labeling buffer on the bench top for thirty minutes. This labeling time was chosen for two reasons: the time scale is kinetically favorable for complete labeling of the peptide (both side chains and backbone); and, fibrils formed at this timescale.

\subsubsection{Thioflavin T Assay.}

The assay was performed using a Molecular Devices M5 spectrophotometer (Molecular Devices, LLC, Sunnyvale, CA). Stock thioflavin T (Sigma Aldrich, St. Louis, MO) was prepared in nanopure water at a concentration of $1.0 \mathrm{mg} \cdot \mathrm{mL}^{-1}$. Peptide samples were prepared as described above. $2.0 \mu \mathrm{L}$ of the stock thioflavin T was spiked into the sample (100 $\mu \mathrm{L}$ total sample volume/well). Sample fluorescence was monitored using $440 \mathrm{~nm}$ excitation and $480 \mathrm{~nm}$ emission wavelengths. Samples were kept at $25^{\circ} \mathrm{C}$ for the duration of the assay. Emission data points were acquired every minute for three hours in each sample buffer (labeling, DHX, and disaggregation). Background fluorescence was obtained from a blank sample that contained no peptide, collected 
simultaneously with the analyte. The background signal did not deviate more than \pm 0.44 in intensity. Each peptide sample was run in triplicate and averaged. The average background signal was subtracted from each data point in the spectrum to obtain the actual fluorescence associated with $\beta$-sheet formation. Signal was normalized to the highest intensity value for each trial.

\subsubsection{Atomic Force Microcopy.}

Peptide solutions were sampled after 0,30 , and 180 minutes of incubation. At each time-point, $5 \mathrm{~mL}$ aliquots of solution were deposited on freshly cleaved mica for 1 minute, washed with $200 \mathrm{~mL}$ of ultrapure water, and dried with a gentle stream of purified air. Each substrate was imaged in air with a Nanoscope $\vee$ MultiMode scanning probe microscope (Veeco, Santa Barbara, CA) equipped with a closed-loop vertical engage J-scanner and operated in tapping mode. Images were acquired with diving board shaped silicon cantilevers with a nominal spring constant of $40 \mathrm{~N} / \mathrm{m}$ and a resonance frequency of $\sim 300 \mathrm{kHz}$. AFM image analysis was performed using Matlab outfitted with the image processing toolbox. (MathWorks, Natick, MA). To distinguish between oligomeric and fibrillar aggregates in AFM images, an aspect ratio (defined as $R_{\mathrm{a}}=r_{1} / r_{2}$, with $r_{1}$ being the largest and $r_{2}$ being the shortest distances across an aggregate) filter was used. ${ }^{31}$ Oligomers were defined as any aggregate at least $1 \mathrm{~nm}$ in height with an aspect ratio less than 2.5 , indicating a globular structure. Fibrils were defined as aggregates greater than $1 \mathrm{~nm}$ in height with an aspect ratio greater than 2.5 , which corresponds to an elongated fibrillar structure. 


\subsubsection{DHX Tandem Mass Spectrometry.}

Samples were prepared as described above, with the addition of a $0.1 \mathrm{mg} / \mathrm{mL}$ bradykinin internal standard $(0.01 \mathrm{mg} / \mathrm{mL}$ final concentration $)$. After labeling, the sample was injected onto an LTQ Velos Pro linear ion trap mass spectrometer (Thermo Scientific, San Jose, CA) using a $0.100 \mathrm{~mm}$ I.D. fused silica capillary at a flow rate of $0.5 \mu \mathrm{L} \cdot \mathrm{min}^{-1}$. Electrospray ionization ESI emitter tips were pulled from untreated fused silica capillary and the ESI potential was maintained at $1.80 \mathrm{kV}$ above the MS entrance orifice $\left(180^{\circ} \mathrm{C}\right)$. Water with $0.100 \mathrm{M}$ ammonium acetate was used as the $\mathrm{DHX}$ buffer. On-line quench and disaggregation after DHX was achieved by acetonitrile with $10 \%$ acetic acid. The DHX and disaggregation buffers were introduced sequentially using low-volume microcross assemblies (Upchurch Scientific) at flow rates of 1.0 and 0.5 $\mu \mathrm{L} \cdot \mathrm{min}^{-1}$, respectively. The sample was exposed to the $\mathrm{DHX}$ buffer at room temperature $\left(25^{\circ} \mathrm{C}\right)$ over a $2.2 \mathrm{in}$. mixing region, corresponding to 17.6 seconds of exposure time. Quench and disaggregation was performed over a 3.5 in. length of capillary, which corresponds to 21.2 seconds of quench and disaggregation. Jorgensen et al. report that back exchange is negligible for a lysine-containing peptide fragment exposed to an acidic quench buffer for under thirty seconds. ${ }^{32}$ Full MS scans as well as ETD MS scans for the most abundant charge state peaks were acquired for the unlabeled sample, the labeled sample, and the DHX sample. Unlabeled samples were prepared in the same manner as the labeled samples, except the solvent was switched to $\mathrm{H}_{2} \mathrm{O}$. In both cases, the disaggregation buffer was used. This provided a direct comparison of ETD mass spectra without the reliance on in silico fragmentation, though this method was used initially to determine peaks of interest. Fully labeled ETD spectra were acquired for each 
charge state without the use of the DHX buffer. lons were accumulated in the trap for 75 $\mathrm{ms}$ with a $4.0 \mathrm{~m} / \mathrm{z}$ window to ensure adequate ion coverage. Each spectrum was averaged for five minutes. Before DHX experiments were performed with the htt peptide, a bradykinin DHX experiment was performed to determine the point at which to collect the spectra. Bradykinin was allowed to incubate in the labeling buffer for thirty minutes. A full MS scan was observed for the fully labeled peptide. The sample was then exposed to the $\mathrm{DHX}$ buffer until the $[\mathrm{M}+\mathrm{H}]^{+}$bradykinin signal did not change significantly (approximately five minutes, data not shown). This value was used to determine the time at which to start acquisition of the htt DHX spectra.

\subsubsection{DHX Data Analysis.}

The centroid $\mathrm{m} / \mathrm{z}$ from each isotopic envelope was selected manually and used as the peak for determining exchange levels. The list generated from ETD-MS of each charge state for the unlabeled samples was cross-referenced with an in silico fragmentation pattern list (c- and z- type ions) generated from Protein Prospector's MS Product tool (http://prospector.ucsf.edu/prospector/cgibin/msform.cgi?form=msproduct). Peaks were assigned to the respective c- or $z$ - ion label if the actual value did not vary from the theoretical by more than $\pm 0.5 \mathrm{~m} / \mathrm{z}$. The peak list generated from the labeled charge states was compared to a theoretical list generated by adding a theoretical total deuterium uptake (sidechain + backbone) to the obtained ETD-MS values. Here, uptake for ion $\mathrm{c}_{\mathrm{i}+1}$ must not be significantly less (-1 deuterium) than $\mathrm{c}_{\mathrm{i}}$. The $\mathrm{DHX}$ peaks were also found manually; however, labels were assigned to each ion if their $\mathrm{m} / \mathrm{z}$ value fell between the unlabeled and labeled value for 
the same ion. As with the labeled sample, $m / z$ values for $c_{i+1}$ could not be less than $c_{i}$. Each figure represents an average of three individual experiments run under identical conditions on separate days. Intrinsic D-to-H exchange rates were calculated for comparison purposes using Sphere

(https://www.fccc.edu/research/labs/roder/sphere/sphere.html). Exchange conditions were set to $\mathrm{pH} 7.0$ with default pKa values used for glutamic acid residues and the Cterminus (4.5 and 3.72 , respectively).

\subsection{Results and Discussion}

\subsubsection{Htt peptide predominantly forms fibrillar aggregates under experimental} conditions.

The aggregation of amyloid-forming proteins is governed by a number of factors including solution $\mathrm{pH}$ and ionic strength as well as protein concentration. Because the goal of the this study was to characterize the structures of aggregates formed by a truncated htt peptide, it was necessary to survey the aggregation process under conditions that would be employed for the HDX-MS analyses, which deviated from established polyQ peptide preparation protocols ${ }^{30}$ and to determine the morphology of the aggregates obtained. Using a thioflavin T (ThT) assay, the aggregation profile was

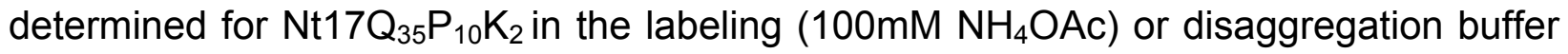
(acetonitrile with $10 \%$ acetic acid, Figure $2.1 \mathrm{~A}$ ). Incubation of $\mathrm{Nt}_{17} \mathrm{Q}_{35} \mathrm{P}_{10} \mathrm{~K}_{2}$ in the disaggregation buffer yielded no enhancement of ThT fluorescence, confirming that the

protein did not aggregate under these conditions. However, initiation of $\beta$-sheet formation occurred within minutes of incubation in the labeling buffer. The aggregation 
rate rapidly increased for approximately the next 25 minutes and decreased thereafter, beginning to plateau at $\sim 80$ minutes. At longer time points (>80 minutes), the ThT fluorescence response associated with incubation of $N t 17 Q_{35} P_{10} K_{2}$ was indicative of a large degree of $\beta$-sheet content, consistent with fibril formation (Figure 2.1A).
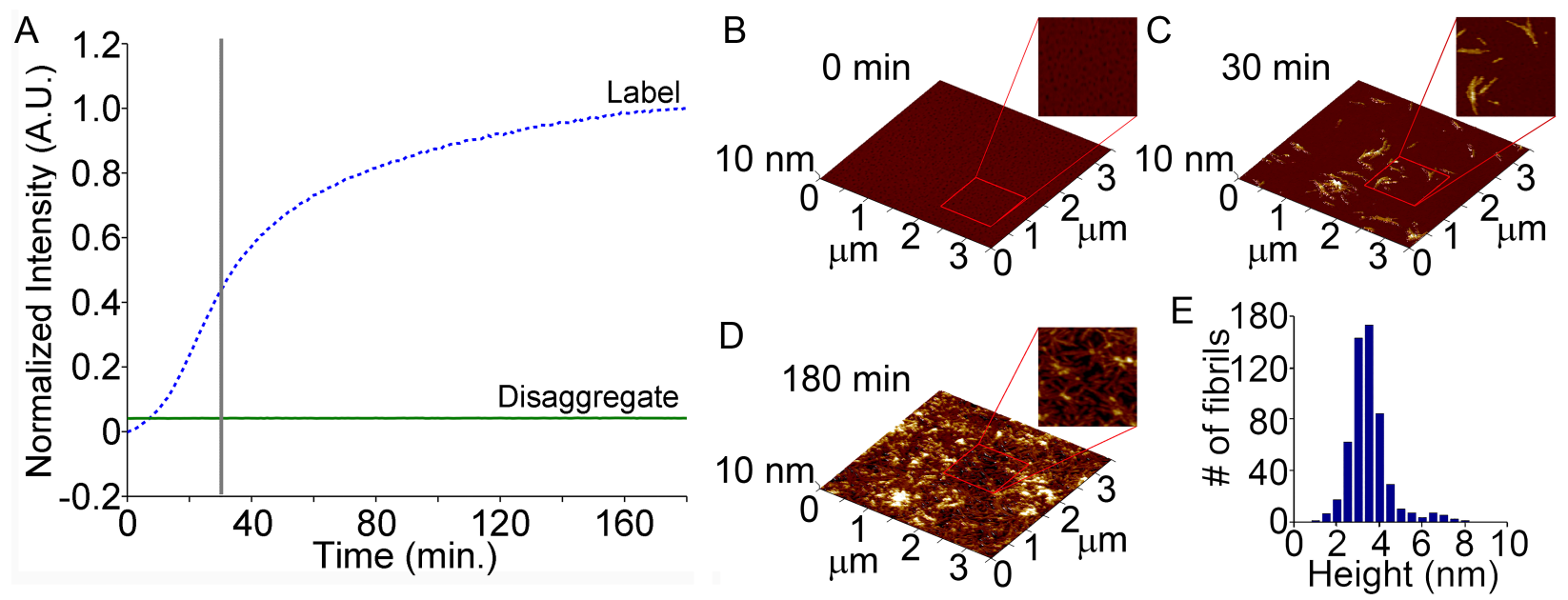

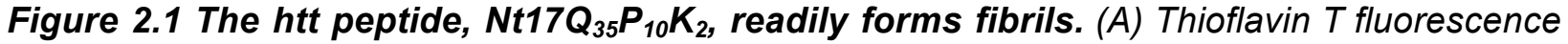
tracked $\boldsymbol{\beta}$-sheet formation of $N t 17 Q_{35} P_{10} K_{2}$, which is indicative of fibril formation. The blue dashed line represents the $N t 17 Q_{35} P_{10} K_{2}$ peptide in the labeling buffer; the solid green line represents the same sample with the addition of the disaggregation buffer. DHX-MS samples were taken at $t=30 \mathrm{~min}$ (gray vertical line). All fluorescence intensities were normalized to the most intense overall value. (B-E): $A F M$ analyses of $N t 17 Q_{35} P_{10} K_{2}$ peptide aggregates. (B) At $t=$ 0 , no fibrils were present; although, a peptide film formed on the substrate. (C) After $30 \mathrm{~min}$, a mix of oligomeric and fibrillar aggregates had formed. (D) At $t=180 \mathrm{~min}$, fibrils completely covered the surface. (E) The average height along the contour of the $N t 17 Q_{35} P_{10} K_{2}$ fibrils was roughly $3.5 \mathrm{~nm}$.

Similar to that performed previously, ${ }^{33}$ a separate experiment was conducted in which a $50 \mu \mathrm{L}$ aliquot of the disaggregation buffer was added to the htt-ThT assay at $\mathrm{t}=25$ minutes (Figure 2.2). A dramatic decrease in fluorescence intensity was observed. The final $\mathrm{pH}$ of the sample after addition of the disaggregation solution was 4.2. Studies on insulin amyloid fibrils show that at $\mathrm{pH} 1.6$, ThT loses affinity for the fibril due to charge repulsion between the amyloid protein and $\mathrm{ThT}^{34}$ Since glutamine residues are uncharged at $\mathrm{pH} 4.2$, no such charge repulsion occurs; therefore, the observed 
decrease in fluorescence intensity is most likely due to a decrease in $\beta$-sheet content and not due to a decrease in ThT affinity for fibrillar polyQ. Similar studies show that ThT assays are effective for uncharged proteins down to $\mathrm{pH} 3.9{ }^{33}$

A separate mass spectrometry experiment was performed on incubated peptide. Samples were prepared in the same manner outlined in the Methods section. After 30 minutes, the sample was split into two equal aliquots. A $100 \mu \mathrm{L}$ aliquot of the disaggregation buffer was added to one sample, while $100 \mu \mathrm{L}$ of ammonium acetate was added to another. A $1.0 \mathrm{mg} / \mathrm{mL}$ bradykinin standard was added to both samples (final concentration: $0.01 \mathrm{mg} / \mathrm{mL}$ ). The samples were centrifuged at $20,800 \mathrm{xg}$ for five minutes to pellet the aggregated peptide. The supernatant was removed and dried down in a centrifugal vacuum concentrator until all solvent was evaporated. Both of the resulting films were reconstituted in 50/50 water/acetonitrile with $0.1 \%$ acetic acid and electrosprayed immediately. The ion intensities of all $N t 17-Q_{35}-P_{10}-K_{2}$ charge states were summed and normalized to the total ion intensity of bradykinin. The Nt17- $\mathrm{Q}_{35}-\mathrm{P}_{10^{-}}$ $\mathrm{K}_{2}$ : Bradykinin ratio for the $\mathrm{NH}_{4} \mathrm{OAc}$ sample was 0.11 , while for the $\mathrm{ACN} / \mathrm{HOAc}$ sample, the ratio was 0.41 ; four-fold more peptide was found in the supernatant of the disaggregated sample versus the control. This suggests that the high-organic, low pH disaggregation buffer dissolves at least part of the aggregated sample, perhaps those that are in an early transient complex. The decrease in $\beta$-sheet content can be associated with partial disaggregation, suggesting a portion of species that are observed in later mass spectrometry experiments originated from an aggregated state. 


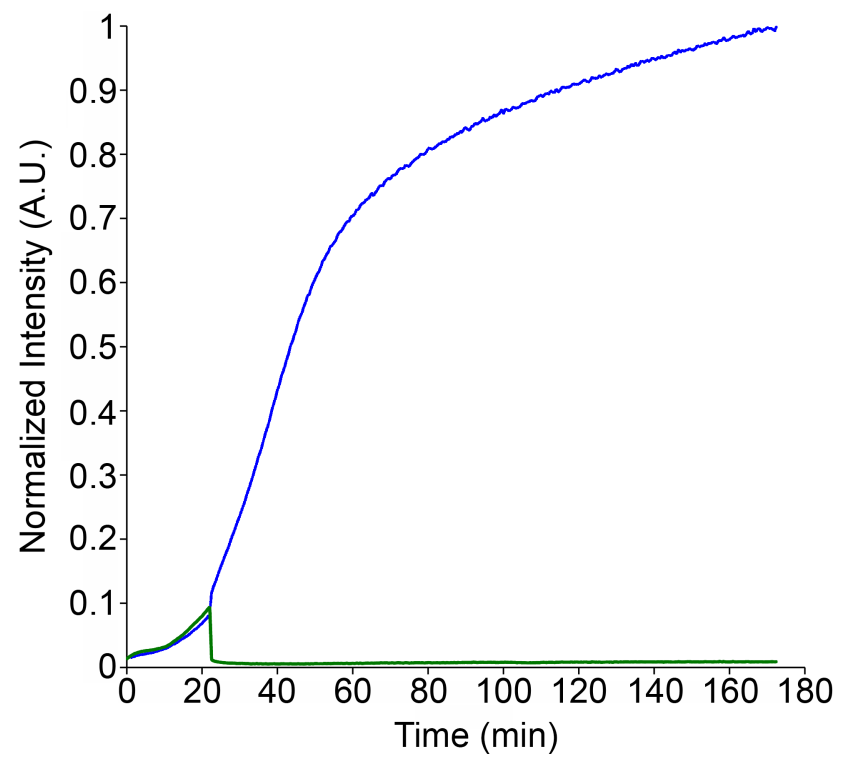

Figure 2.2. Disaggregation of $N t 17 Q_{35} P_{10} K_{2}$ by addition of ACN/HOAC (90/10). Blue line: thioflavin $T$ fluorescence of $N t 17 Q_{35} P_{10} K_{2}$ in NH4OAc buffer. Green line: disaggregated $N t 17 Q_{35} P_{10} K_{2}$. All experiments were performed in triplicate and averaged.

To characterize aggregate

morphology, the $\mathrm{Nt} 17 \mathrm{Q}_{35} \mathrm{P}_{10} \mathrm{~K}_{2}$ incubations were sampled at several time-points for AFM analysis. Despite the short lag phase for the initiation of aggregation in the labeling buffer, the initial prep was predominately unaggregated as determined by AFM analysis of freshly prepared peptide, i.e., at time-point 0 minutes (Figure 2.1B). Freshly prepared peptide formed a perforated film on mica, indicative of soluble protein depositing onto the surface. Consistent with the ThT assay, $\mathrm{Nt} 17 \mathrm{Q}_{35} \mathrm{P}_{10} \mathrm{~K}_{2}$ formed a significant number of fibrils after 30 minutes of incubation in the labeling buffer (Figure 2.1C), and the number of fibrils increased at 180 minutes, covering the entire surface in a dense mesh (Figure 2.1D). While there were a few globular aggregates observed at 30 minutes that represent oligomers, fibrils were the dominant species (> $98 \%$ by total volume of deposited peptide in aggregates as determined by AFM image analysis, Figure 2.3). After 30 minutes of incubation in the labeling buffer, the average height along the contour of a fibril was predominately 3-4 $\mathrm{nm}$; however, this height increased to $5-8 \mathrm{~nm}$ as fibrils bundled together (Figure 2.1E). 


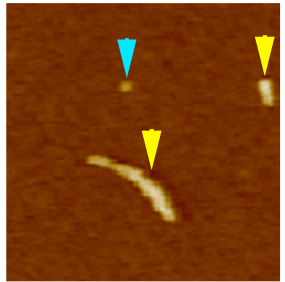

$250 \mathrm{~nm}$

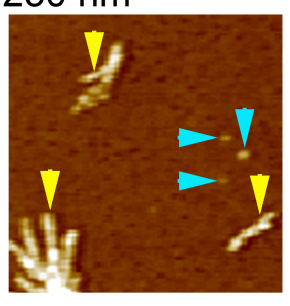

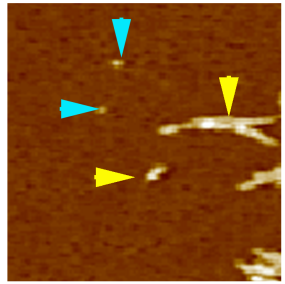

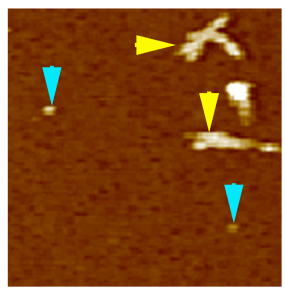

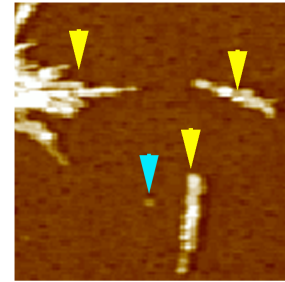
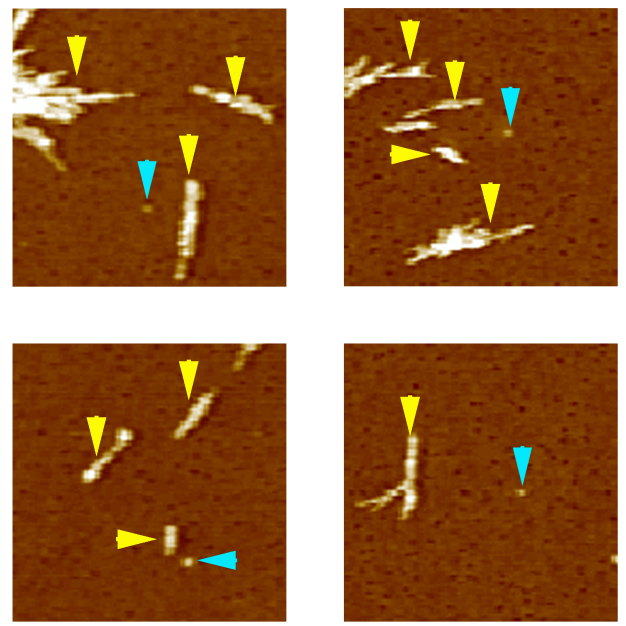
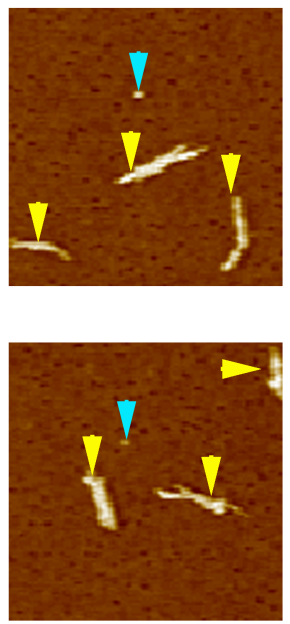

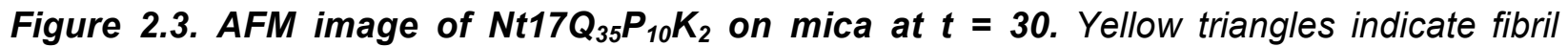
structures; blue triangles indicate oligomeric structures. Each panel represents a different part of the sample. Percent oligomer content was calculated to be less than $2 \%$.

It is important to note that the kinetics of peptide aggregation in this study was accelerated relative to previous studies with similar peptides ${ }^{4}$ due, in part, to an increased peptide concentration and potentially differences in the aggregation buffer. It is known that aggregation kinetics of polyQ and htt exon1 increases with concentration. ${ }^{35}$ It is also well-known that sample preparation and buffer conditions can greatly influence the formation of amyloid structures, both kinetically and morphologically. ${ }^{30}$ The aggregation profile associated with incubation in both buffers results in important implications for the following structural analysis using DHX-MS-MS. The high organic, low pH disaggregation buffer, the same used in the initial ThT experiments, prevented aggregation over the experimental time period (Figure 2.1A), which is consistent with high organic solvents traditionally being used as protein denaturants in bottom-up proteomic experiments. $\beta$-sheet character is non-existent with the addition of the disaggregation buffer. This buffer also served to quench the exchange reaction. The electrospray ionization process occurred approximately twenty 
seconds after disaggregation. This prevented undesired back-exchange of newly exposed exchange sites similar to that shown in other studies using standard peptides. $^{36}$

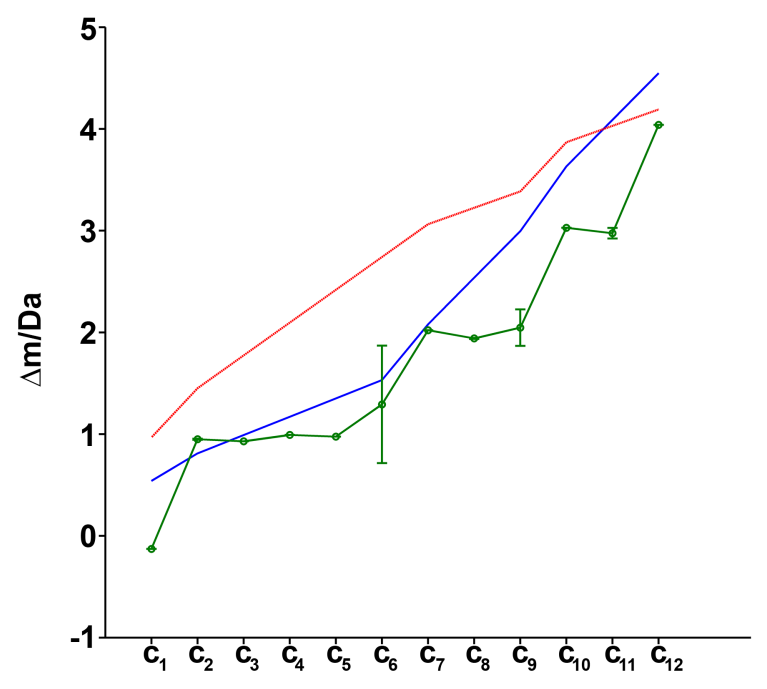

Figure 2.4. Scrambling controls. Red line: 100\% scrambling, Blue line: 0\% scrambling, Green line: experimental data. Nearly full backexchange in the $\mathrm{N}$-terminal half of the peptide and retention in the C-terminal half is observed.
Control experiments were conducted to insure no deuterium scrambling occurred in the mass spectrometer (Figure 2.4). The peptide KKDDDDDIIKIIK was purchased from Genscript, Inc. (Piscataway, NJ) and treated according to protocols outlined by Zehl et al. It should be noted that Zehl et al. used a hexa-aspartate tract, while this control utilizes a pentaaspartate tract. Briefly, peptide was incubated in $99 \% D_{2} \mathrm{O}$ for 18 hours at $25^{\circ} \mathrm{C}$ to ensure full deuteration. This peptide contains a fast-exchanging dilysine pentaaspartate tract at the $\mathrm{N}$-terminal region and a slow-exchanging IIKIIK tract at the $\mathrm{C}$ terminal end. It is assumed that upon introduction to a DHX solution in the absence of deuterium scrambling, that the $\mathrm{N}$-terminal region will back-exchange quickly, while the C-terminal portion will retain its deuterium. In the case of scrambling, one would observe even distribution of deuterium to all available sites. The peptide was subjected to the same on-line $\mathrm{DHX}$ procedure as the $\mathrm{Nt} 17 \mathrm{Q}_{35} \mathrm{P}_{10} \mathrm{~K}_{2}$ peptide, at the same flow rates. Our experimental data matches closely with that of a $0 \%$ scrambling model. 
Additionally, we do not observe deuterium retention on the N-terminus, which is also indicative of deuterium scrambling. Therefore, we can conclude, that under our experimental conditions, no deuterium scrambling is occurring.

\subsubsection{Full MS of Labeled $N t_{17} Q_{35} P_{10} \underline{K}_{2}$ Demonstrates Six Distinct Charge States.}

The ESI mass spectrum of native, aggregated $(t=30$ minutes, Figure $2.6 \mathrm{~A}$ and B) $\mathrm{Nt} 17 \mathrm{Q}_{35} \mathrm{P}_{10} \mathrm{~K}_{2}$ peptide after introduction of the disaggregation buffer shows the generation of six charge states $\left([\mathrm{M}+5 \mathrm{H}]^{5+}\right.$ to $[\mathrm{M}+10 \mathrm{H}]^{10+}$ (see Figure 2.5 for on-line $\mathrm{DHX}$ schematic).

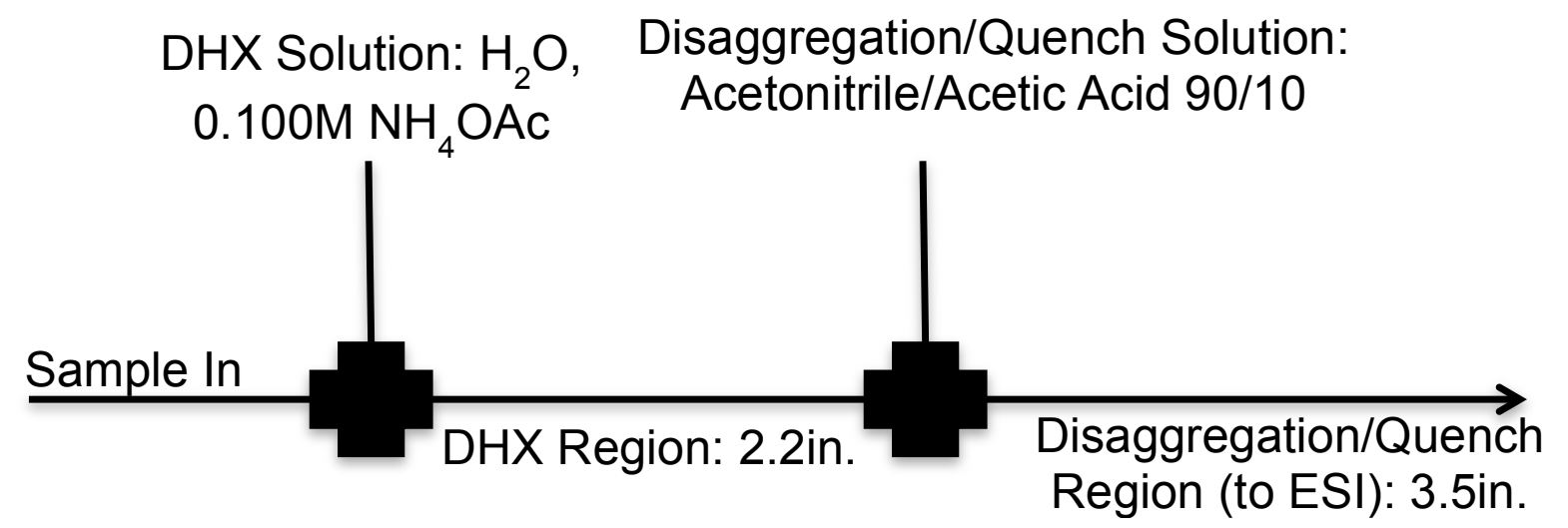

Figure 2.5. On-line DHX schematic. Untreated fused silica capillary was used for all mass spectrometry experiments. Sample is pumped into the first microcross assembly at a rate of $0.5 \mu \mathrm{L} / \mathrm{min}$. DHX solution was introduced at a flow rate of $1.0 \mu \mathrm{L} / \mathrm{min}$, resulting in a total $D H X$ time of 17.7 seconds along the 2.2in. capillary. The inlet for this solution was capped for experiments in which no wash was used. The disaggregation/quench solution was introduced at the second microcross at a flow rate of $0.5 \mu \mathrm{L} / \mathrm{min}$, resulting in 21.2 seconds of disaggregation. The end of the region was pulled into an ESI emitter and used for nanospray ESI-MS.

The maximum charge state observed in the ESI distribution was the $[\mathrm{M}+10 \mathrm{H}]^{10+}$ species. The $[\mathrm{M}+8 \mathrm{H}]^{8+}$ was the most intense feature in the ESI charge state distribution, and a significant decrease in intensity was observed for the $[\mathrm{M}+5 \mathrm{H}]^{5+}$ charge state. Of 


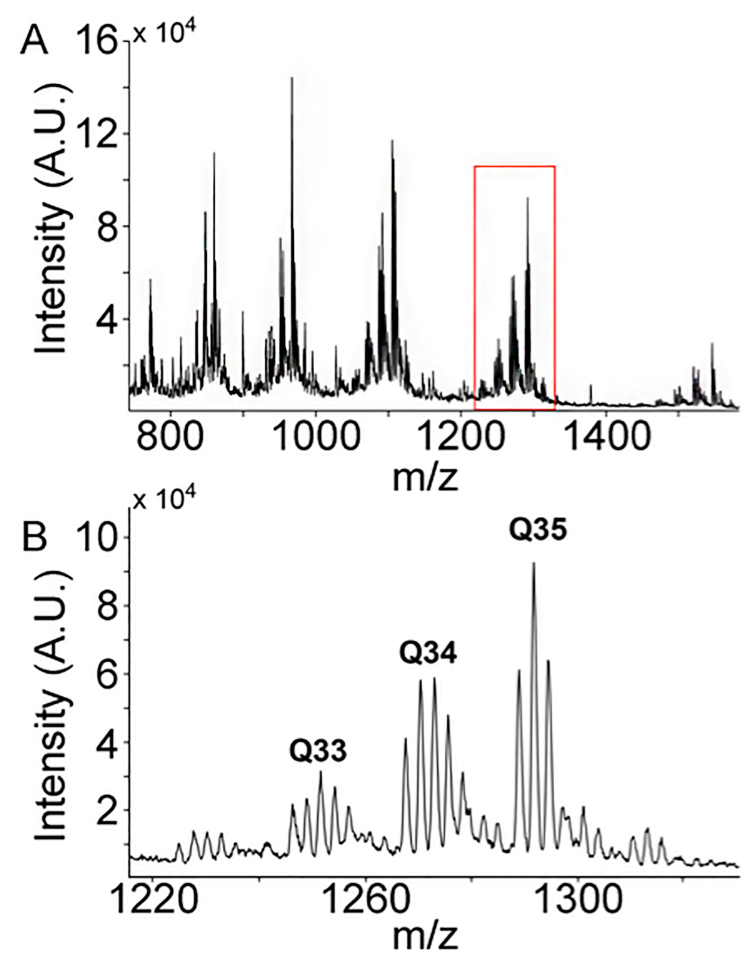

Figure 2.6. Full mass spectrum of the unlabeled $N t 17 Q_{35} P_{10} K_{2}$ shows six accessible charge states. (A) Six charge states are observed for the sample ranging from $[\mathrm{M}+5 \mathrm{H}]^{5+}$ to $[\mathrm{M}+10 \mathrm{H}]^{10+}$. (B) Each charge state manifests as a multiplet with the most intense peak representing a $Q_{35}$ chain. these, only three were used for by residue fragmentation analysis $\left([\mathrm{M}+7 \mathrm{H}]^{7+}\right.$ to $[\mathrm{M}+9 \mathrm{H}]^{9+}$ ) because the higher charge states are more amenable to the production of informative ETD fragmentation spectra. ${ }^{37,38}$ The $[\mathrm{M}+10 \mathrm{H}]^{10+}$ was omitted from ETD studies due to poor ion statistics and low signal. Each charge state was observed as a multiplet peak in the ESI mass spectrum (Figure 2.6B). Comprising peaks differed by $\sim 128 \mathrm{Da}$, indicating that these species correspond to peptides with truncated polyQ domains (i.e., $<35 \mathrm{Q}$ ) and were presumably remnants of peptide

synthesis. For each charge state, only the peak corresponding to peptides containing exactly $35 \mathrm{Q}$ repeats was analyzed; however, heterogeneous aggregation (e.g., 35Q interaction with $34 \mathrm{Q}$ or $33 \mathrm{Q}$ ) cannot be ruled out. Such a scenario is possible, as EPR analysis of fibril structure of similar htt peptides demonstrated that the polyQ domain does not need to be in register within the fibril structure ${ }^{17}$ Ions associated with single peptide sequences are observed to contain varying numbers of solvent adduct molecules, $+17 \mathrm{Da}$, most likely $\mathrm{NH}_{3}$, as well as some oxidation on $\mathrm{M} 1$ as shown in 
Figure 2.6B. In each case, the non-adducted, non-oxidized ion was selected for ETD analysis after HDX and DHX.

\subsubsection{Protein HDX and DHX exchange levels.}

For the experiments described below, the $\mathrm{Nt}_{17} \mathrm{Q}_{35} \mathrm{P}_{10} \mathrm{~K}_{2}$ peptide was incubated in the labeling buffer for $\sim 30$ minutes prior to initiation of MS measurements. To determine the maximum level of deuterium incorporation, the incubation solution was electrosprayed directly into the mass spectrometer. Table 2.1 lists the numbers of deuterium atoms that were incorporated into the protein ions for each of the observed charge states.

\begin{tabular}{c|c|c|c|c|c} 
Charge $^{a}$ & D Uptake $^{b}$ & D Retained $^{c}$ & D Removed $^{d}$ & $\begin{array}{c}\text { D Retained, } \\
\text { Nt17 }^{e}\end{array}$ & $\begin{array}{c}\text { D Retained, } \\
\text { Poly }^{f}\end{array}$ \\
\hline 9 & 119.97 & 39.69 & 80.28 & 9.58 & 30.11 \\
8 & 116.64 & 44.00 & 72.64 & 9.97 & 34.03 \\
7 & 118.72 & 42.84 & 75.88 & 9.83 & 33.01 \\
6 & 120.06 & 48.00 & 72.06 & - & - \\
5 & 118.75 & 42.10 & 76.65 & - & - \\
Average & $118 \pm 1$ & $43 \pm 3$ & $75 \pm 3$ & $9.8 \pm 0.3$ & $32 \pm 2$
\end{tabular}

Table 2.1. Total Deuterium Uptake and Retention by Charge State ${ }^{a}$ Charge states of each peak were obtained using a Thermo Q Exactive FTMS under the same source conditions as the $L T Q$ Velos. ${ }^{b}$ Deuterium uptake calculated by subtraction of the unlabeled mass from the labeled mass for each charge state. ${ }^{\circ}$ Deuterium retention after $D H X$ and quench step calculated from subtraction of the native peptide mass from the mass after $D H X .{ }^{d}$ Deuterium removed by $D H X$ calculated by subtraction of the mass after DHX from the fully labeled mass. ${ }^{e}$ Deuterium retained in the Nt17 region calculated from the $c_{15}$ ion. ${ }^{f}$ Deuterium retained in the poly $Q$ region calculated by subtraction of $D$ Retained, Nt17 from total $D$ Retained.

On average, the maximum number of deuterium atoms incorporated into the molecule was $118 \pm 1$, corresponding to $\sim 85 \%$ of potential exchange sites. There are several possible explanations that would explain the lack of complete deuteration. One is that upon exposure to the quench/disaggregation buffer, rapidly exchanging side 
chain hydrogens undergo back exchange. It is noted that previous HDX exchange studies of amyloid- $\beta$, a combined wash/disaggregation buffer was used that consisted of water/acetonitrile/formic acid (50:50:0.1) ${ }^{36}$ In these experiments, the solution served to quench the HDX reaction as well as disaggregate the sample. However, the latter buffer contains a significant source of protons that could potentially contribute to back exchange. To decrease artifactual back exchange in the present study, a quench/disaggregation buffer resulting in an ESI tip proton:deuteron ratio of $\sim 1: 40$ was employed after DHX. That said, back exchange cannot be completely ruled out in explaining the deuterium uptake deviations. A second explanation is that solvent exposure decreases due to inter- or intra-molecular interaction for some residues which would cause protection of some side-chain and backbone hydrogens.

Exposure of the htt peptide, via an online wash, to an aqueous back-exchange buffer (100mM NH${ }_{4} \mathrm{OAc}$ ) followed by the quench/disaggregation buffer resulted in removal of $\sim 64 \%$ of the deuterium atoms (Table 2.1 ). The deuterium retention suggests a high degree of interaction at $t=30$ minutes, some of which could be due to aggregation. The observed peptide cannot unambiguously be differentiated from originating from an aggregated or monomeric state; however, a four-fold increase in monomeric peptide is observed in a filtered sample containing the disaggregation buffer relative to a filtered, aggregated control (see Figure 2.2). 
from maximum uptake.

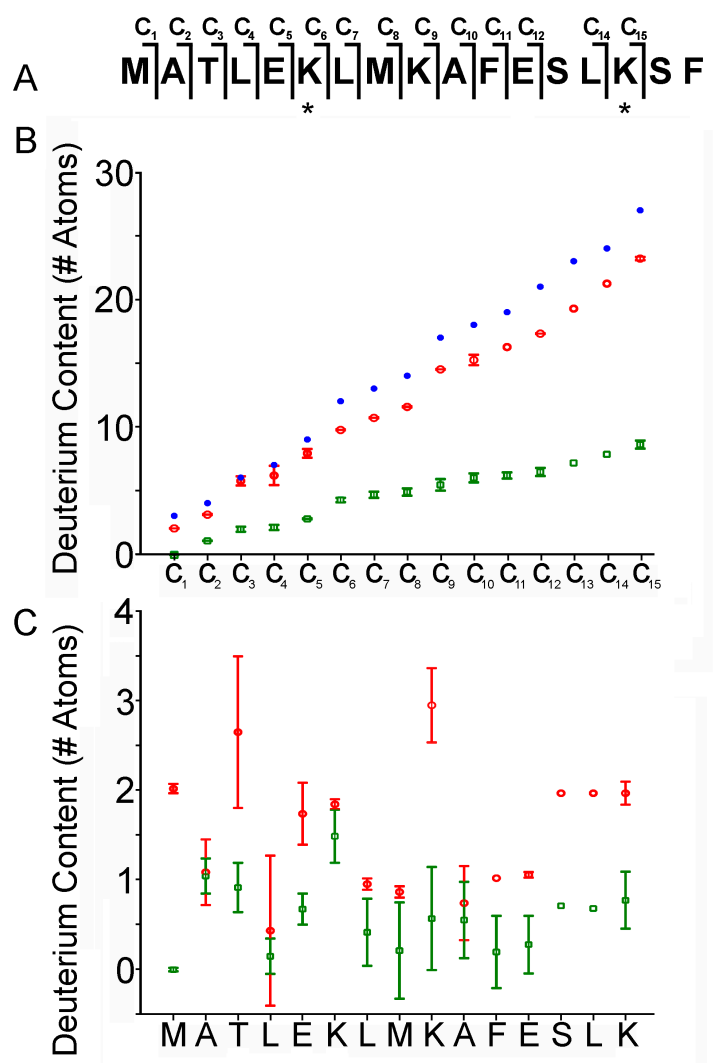

Figure 2.7. N-terminal region of the $N t 17 Q_{35} P_{10} K_{2}$ peptide is solvent exposed and contains secondary structure in the aggregated form. $(A)$ Fragmentation pattern for the Nt17 region. (B) Total deuterium uptake by ion before (red circles) and after (green squares) DHX. Blue markers represent full theoretical deuterium incorporation for each ion. (C) Deuterium uptake and retention by residue. Red circles are calculated uptake by residue. Green squares are calculated retained deuterium by residue. Error bars represent a propagation of error originating from the average of three MS measurements per charge state. The error was carried through all arithmetic.
Subjecting select protein charge states

$(+7$ to +9$)$ to ETD yields primarily c-type ions.

These ions comprise a nearly complete

series from the $\mathrm{N}$-terminus containing each

residue in the $\mathrm{N}$-terminal sequence at the

cleavage site. Site characterization is

focused on the c-ion series for two reasons:

the $\mathrm{N}$-terminal region is implicated in

modulating aggregation kinetics and

promoting specific aggregate forms ${ }^{27}$ and the large polyP tract at the $\mathrm{C}$-terminal region affords no information because it does not readily fragment via ETD. Each c-ion that is created contains a reduced carbonyl group that originates from a charge (basic) site. ${ }^{39}$ It is noted that in HDX experiments, this means donation of a proton or, more likely, a deuteron. That said, in comparisons to the theoretical maximum uptake (Figure 2.7), this site is added to the sum of exchange sites for each ion. 
The average deuterium uptake (HDX experiments) of each ion in the c-ion series increases linearly, with some deviation from theoretical (Figure 2.7B). The $\mathrm{c}_{1}$ ion has a theoretical uptake that is one higher than that expected for the acetylated amino acid residue due to the charge site. Thereafter, the charging deuteron is not counted. That is, each subsequent theoretical uptake value is calculated by adding the number of exchangeable side chain and backbone hydrogen atoms to the preceding ion. For example, $c_{1}(M)$ has a theoretical uptake of three deuterium atoms (a backbone, a reduced carbonyl from the ETD mechanism ${ }^{40}$, and a charge). $\mathrm{C}_{2}$ (MA) has a theoretical uptake equal to $c_{1}$ plus an additional backbone site.

For the first 5 residues, the deuterium uptake levels and deuterium increase per ion are very near that expected for complete uptake (Figure 2.7B). At the $\mathrm{c}_{6}$ ion a greater deviation from the maximum theoretical values is observed. From $c_{6}$ to $c_{11}$ deuterium increase per residue is very similar to the theoretical values for complete uptake. The $c_{12}$ ion, like the $c_{6}$ ion, exhibits an increase in deviation from the experimental that is preserved to the $\mathrm{c}_{15}$ ion. Overall, it appears that, with the exception of a few residues, complete deuterium uptake is observed across the first 15 amino acid residues. The deviations from the maximum uptake values at residues $\mathrm{K} 6$ and $\mathrm{E} 12$ could result from one of two different phenomena. The first is that during the aggregation process, these residues become involved in important interactions resulting in protection of hydrogens and no deuterium uptake is observed. The second explanation is that upon addition of quench/disaggregation buffer, DHX occurs resulting in the incorporation of hydrogens. Even though the proton:deuteron ratio is low $(\sim 1: 40)$ 
at the ESI tip for these HDX experiments (see above), the latter explanation could explain the observed results.

The deuterium uptake data can also be described in terms of the amount of deuterium incorporation per amino acid residue (Figure 2.7C). The uptake by residue is determined by subtracting the uptake value (Figure 2.7B) of the immediately preceding $\mathrm{c}$ ion from that of the ion of interest. In general polar residues (with heteroatom exchange sites) exhibit significantly more than one deuterium uptake per residue. Residues exhibiting the greatest uptake include $\mathrm{M} 1, \mathrm{~T} 3, \mathrm{~K} 9$, and $\mathrm{K} 15$. It is noted that we do not consider $\mathrm{S} 13$ because the $\mathrm{c} 13$ ion is not observed and exchange values for $\mathrm{S} 13$ (and L14) are computed as averages between adjacent residues as performed previously ${ }^{41}$. Interestingly, the polar E12 residue exhibits a relatively low uptake value. As mentioned above, this deviation could result from back exchange or from an important interaction associated with aggregates. The remaining non-polar residues in this region of the protein all show complete uptake of 1 deuterium with the exception of L4 (Figure 2.7C). The reason for incomplete uptake is not known; however, the larger error associated with this measurement is noted, indicating that ion statistics could contribute to the skewed result.

\subsubsection{Deuterium protection by residue - $D$-to- $H$-exchange $(D H X)$ demonstrates}

protection in Nt17.

Increased understanding of the structure of the $\mathrm{N}$-terminal region of the htt protein as well as the role of certain residues within aggregate structure is obtained from ETD of the DHX protein ions. The $c_{1}$ ion shows that essentially all of the deuteriums 
have been removed by DHX (Figure 2.7B, green squares). The $\mathrm{c}_{2}$ ion shows protection of $\sim 1$ deuterium. From the $c_{2}$ ion to the $c_{15}$ ion some protection of deuteriums is observed increasing from $\sim 1$ deuterium to $\sim 9$ deuteriums by $c_{15}$. The increase in retained deuteriums (Figure $2.7 \mathrm{~B})$ is relatively constant ( $\sim 0.5$ deuteriums per residue) with the exception of the transition from the $c_{5}$ ion to the $c_{6}$ ion ( 1.5 deuteriums). After $\mathrm{C}_{6}$ there is a gradual increase until $\mathrm{c}_{12}$ with a slight increase from $\mathrm{c}_{12}$ to $\mathrm{C}_{15}$. It is noted that the $c_{13}$ and $c_{14}$ ions are computed as averages and so it may be possible that the slight uptake in deuterium protection is evenly distributed across the $c_{13}$ to $c_{15}$ residues; however, it is also possible that there is a significant increase in protection at the $c_{15}$ residue.

The DHX provides confirmatory and additional information regarding protein structure. DHX-ETD-MS experiments suggests that the $\mathrm{N}$-terminal region is solventexposed in the aggregated form as the $\mathrm{N}$-terminal residue $(\mathrm{M})$ and unprotected sidechain deuterium atoms from other residues are removed by solvent dilution into $\mathrm{H}_{2} \mathrm{O}$. If the $\mathrm{N}$-terminal region were concealed in the center of the aggregate, a higher degree of deuterium retention may be expected; that is, retention would be closer to theoretical backbone values due to decreased solvent accessibility and intermolecular and intramolecular $\mathrm{H}$-bonding. However, the observed deuterium retention indicates that $\mathrm{Nt17}$ is located, at least partially, on the periphery of the aggregate structure.

The decreased protection of backbone amide hydrogens in the $\mathrm{N}$-terminal region stands in contrast to the protection observed in the remaining portion of the protein. Approximately ten deuterium atoms are retained in the $\mathrm{Nt} 17$ region of the peptide, while an average of 43 are retained over the entire peptide. Because proline does not contain 
exchangeable hydrogens, the remaining deuterium must reside in the poly $Q$ region (Table 1). Assuming that upon disaggregation, the polyQ sidechains rapidly back exchange, the protection of amides must be near that of unity, higher than that observed for the N-terminal region. In summary, although our ability to probe the polyQ region was limited as many of the associated fragments were outside the range of the ion trap, the available data conforms to the notion that the polyQ domain forms a rigid core in the fibril, which is further supported by both the ThT and AFM data. Such a scenario is consistent with multiple proposed structures for polyQ packing in fibrils. ${ }^{8,26,}$ 42,43

\subsubsection{HDX and DHX by residue show increased protection on lysine residues.}

A number of polar residues exhibit increased deuterium protection relative to the 0.6 average deuterium retention value (Figure $2.7 \mathrm{C}$ ). It is instructive to consider whether or not this may result from stabilizing interactions for the aggregate structure. The residue that exhibits the most protection is $\mathrm{K} 6$ (Figure 2.7C). As noted above, uptake at this residue is approximately two deuterium less than theoretical (Figure 2.7B). The deuterium that is incorporated is nearly all retained in the $\mathrm{DHX}$ experiment, as only $\sim 0.6$ deuteriums back exchange for hydrogens (Figure 2.7C). This is an important observation because deuterated lysine side chains in Nt17 have been shown to quickly back-exchange in solution. ${ }^{8,44}$ Therefore, this observation may indicate a critical role for $\mathrm{K} 6$ in stabilizing aggregate structure. In contrast, nearly full uptake for the K9 residue is observed with subsequent back-exchange to an $\sim 0.6$ deuterium average suggesting that this side chain is rapidly back-exchanged and not involved in any interaction. Other 
residues that exhibit deuterium protection levels that are greater than the 0.6 average include T3 and K15. Although, the protection levels for these two residues are less pronounced than that of $\mathrm{K} 6$, the potential roles of these residues warrant further investigation. It is important to note that the $\mathrm{S} 13$ residue protection is obtained from the average value assigned to the $c_{13}$ ion and should be treated with caution. Intrinsic exchange rates of the selected residues were calculated. Rapid back-exchange of K9 is not surprising, since its intrinsic D-to-H exchange constant is $12.3 \mathrm{sec}^{-1}$, nearly the same as $\mathrm{A} 2$, which nearly back-exchanges completely. K6 and K15, however, have exchange constants that are much closer in value (6.7 and $5.8 \mathrm{sec}^{-1}$, respectively). These are on nearly the same level as M8, and faster than E12, both of which nearly back-exchange entirely. Therefore, we can conclude, with a fair degree of certainty, that K6 and possibly K15 are truly involved in a stabilizing interaction.

For comparison purposes, the DHX-ESI-MS process was performed at $\mathrm{t}=0$

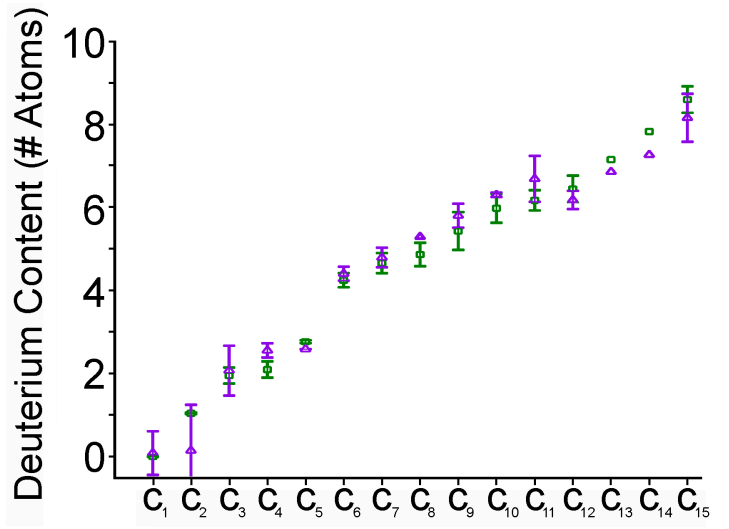

Figure 2.8. The Nt17 region is structured early in the aggregation process. Two DHX time points were sampled: $t=0$ (purple triangles) and $t=30$ (green squares). No significant differences are observed in the retention by ion. minutes (Figure 2.8). Uptake by ion follows the same general trend as the $t$ $=30$ minutes time point, providing evidence for a structured region between $\mathrm{c}_{2}$ and $\mathrm{c}_{15}$. While this data does more closely represent an early aggregate form than the $t=30$ minutes experiment, it should be noted that this is not a 'true' initial time point; it is impossible to account for aggregation that occurs in the capillary leading to the mass 
spectrometer and during the collection of ETD data. As such, it is likely that a conformer 'ensemble' is again sampled; the peptide was sampled closer to the $t=5$ minutes time point. A degree of $\beta$-sheet formation is evident in the ThT curve (Figure 2.1A). The data suggest that aggregates formed at earlier times are structurally similar to those observed to be produced from the more advanced fibrils.

\subsubsection{Comparison of the structural findings.}

One of the central findings of this work is the key role of lysine residues, K6 and $\mathrm{K} 15$, in the Nt17 tract. As it has been shown that a-helical structure in Nt17 is conserved in oligomeric precursors to fibrils, ${ }^{4,8}$ it is notable to consider whether or not similar intermolecular interactions at these two residues also play a role in oligomer formation. The lysine residues in question lie at the "edge" of the hydrophilic region of the amphipathic helix. In the arrangement of helices in the proposed tetrameric oligomer, ${ }^{8}$ $\mathrm{K} 6$ and $\mathrm{K} 15$ could provide a tethering effect that drives formation and stabilizes the structure. Recent work by Michalek et al. suggests exposure of the lysine residues when the $\mathrm{N}$-terminal domain is anchored to a lipid substrate. It is suggested that these residues can participate in an antiparallel interhelical dimer through $\mathrm{K} 9-\mathrm{E} 12$ and $\mathrm{K} 6$ $\mathrm{S} 16 .{ }^{45}$ From these data it would appear that the $\mathrm{K} 6-\mathrm{S} 16$ interaction is the most likely, though the $c_{16}$ peptide fragment fell outside the range of our mass spectrometer.

Several studies have shown that two $S$ to $D$ mutations, which mimic phosporylation, in Nt17 retard aggregation. ${ }^{21,22}$ Trypsin proteolysis of wild type peptide resulted in cleavage of $\mathrm{Nt} 17$ only at the $\mathrm{K} 6$ site, supporting the notion that further residues are protected in the fibril structure and that these residues may play a larger 
role in promoting the formation of specific aggregate forms. Furthermore, introduction of the $S$ to $D$ mutations resulted in cleavage at $K 9,{ }^{21}$ increasing solvent exposure of these residues. These $S$ to $D$ mutations could induce a critical interaction with the neighboring lysine residues that destabilizes precursor structure, and thus, prevents aggregation.

As the Nt17 domain has known lipid-binding properties, ${ }^{7,14,46,47,11,45}$ the availability and orientation of the Nt17 in an aggregate form could dictate if htt aggregates interact with subcellular membranes directly. For example, a recent study using htt-exon1 constructs demonstrated that pre-aggregation of a htt-exon1 protein into fibrils resulted in limited to no interaction with lipid bilayers, ${ }^{15}$ which suggests that the specific residues that facilitate lipid binding in htt are not exposed in the fibril structure. Although further study is warranted, these residues may also be involved in interactions that promote and stabilize htt aggregates in the absence of lipids.. The data presented in the current study, when taken in the context of previously published work on a preaggregated form of the same model peptide in the presence of a lipid bilayer, suggests that the residues that stabilize helix-lipid interaction are unavailable at the time of injection onto a bilayer. It is proposed that the lack of membrane insertion observed by Burke et al. is due to the stabilizing charged residues being unavailable in the aggregated form; our data show that $\mathrm{K} 6$ and $\mathrm{K} 15$ are unavailable, and thus will not stabilize a lipid interaction.

This study represents the first time top down mass spectrometry and isotopic labeling has been applied to structural characterization of huntingtin peptide aggregates. The data show that the 17 residue $\mathrm{N}$-terminal region of htt is at least partially solvent-exposed in the aggregated form. The experimental findings implicate 
several hydrophilic residues in the amino terminal tract of huntingtin that could be involved in critical inter- or intra-molecular interactions, specifically K6 and K15. The data points to several novel interactions in the $\mathrm{N}$-terminal domain that can be targeted in future chemical labeling studies, and is the first to show the importance of lysine residues in huntingtin aggregation. Unlike the bottom-up approach, the method outlined in the current study does not require time-consuming proteolysis that can lead to loss of site-specific information and aggregation of the analyte. Most importantly, the study demonstrates a viable technique application for obtaining meaningful qualitiative structural information on a low-resolution instrument for a relevant biophysical system that has not yet been well-characterized. 


\subsection{References}

(1) The Huntington's Disease Collaborative Research Group (1993) A NOVEL GENE CONTAINING A TRINUCLEOTIDE REPEAT THAT IS EXPANDED AND UNSTABLE ON HUNTINGTONS-DISEASE CHROMOSOMES, Cell 72, 971-983.

(2) Difiglia, M., Sapp, E., Chase, K. O., Davies, S. W., Bates, G. P., Vonsattel, J. P., and Aronin, N. (1997) Aggregation of huntingtin in neuronal intranuclear inclusions and dystrophic neurites in brain, Science 277, 1990-1993.

(3) Penney, J. B., Vonsattel, J. P., MacDonald, M. E., Gusella, J. F., and Myers, R. $H$. (1997) CAG repeat number governs the development rate of pathology in Huntington's disease, Annals of Neurology 41, 689-692.

(4) Jayaraman, M., Kodali, R., Sahoo, B., Thakur, A. K., Mayasundari, A., Mishra, R., Peterson, C. B., and Wetzel, R. (2012) Slow Amyloid Nucleation via alphaHelix-Rich Oligomeric Intermediates in Short Polyglutamine-Containing Huntingtin Fragments, Journal of Molecular Biology 415, 881-899.

(5) Dlugosz, M., and Trylska, J. (2011) Secondary Structures of Native and Pathogenic Huntingtin N-Terminal Fragments, Journal of Physical Chemistry $B$ 115, 11597-11608.

(6) Mishra, R., Jayaraman, M., Roland, B. P., Landrum, E., Fullam, T., Kodali, R., Thakur, A. K., Arduini, I., and Wetzel, R. (2012) Inhibiting the Nucleation of Amyloid Structure in a Huntingtin Fragment by Targeting alpha-Helix-Rich Oligomeric Intermediates, Journal of Molecular Biology 415, 900-917.

(7) Atwal, R. S., Xia, J., Pinchev, D., Taylor, J., Epand, R. M., and Truant, R. (2007) Huntingtin has a membrane association signal that can modulate huntingtin aggregation, nuclear entry and toxicity, Human Molecular Genetics 16, 26002615.

(8) Sivanandam, V. N., Jayaraman, M., Hoop, C. L., Kodali, R., Wetzel, R., and van der Wel, P. C. A. (2011) The Aggregation-Enhancing Huntingtin N-Terminus Is Helical in Amyloid Fibrils, Journal of the American Chemical Society 133, 45584566.

(9) Kelley, N. W., Huang, X. H., Tam, S., Spiess, C., Frydman, J., and Pande, V. S. (2009) The Predicted Structure of the Headpiece of the Huntingtin Protein and Its Implications on Huntingtin Aggregation, Journal of Molecular Biology 388, 919927.

(10) Kim, M. W., Chelliah, Y., Kim, S. W., Otwinowski, Z., and Bezprozvanny, I. (2009) Secondary Structure of Huntingtin Amino-Terminal Region, Structure 17, 1205-1212.

(11) Michalek, M., Salnikov, E. S., Werten, S., and Bechinger, B. (2013) Membrane Interactions of the Amphipathic Amino Terminus of Huntingtin, Biochemistry 52, 847-858.

(12) Rossetti, G., Cossio, P., Laio, A., and Carloni, P. (2011) Conformations of the Huntingtin $\mathrm{N}$-term in aqueous solution from atomistic simulations, Febs Letters 585, 3086-3089.

(13) Nucifora, L. G., Burke, K. A., Feng, X., Arbez, N., Zhu, S. S., Miller, J., Yang, G. C., Ratovitski, T., Delannoy, M., Muchowski, P. J., Finkbeiner, S., Legleiter, J., Ross, C. A., and Poirier, M. A. (2012) Identification of Novel Potentially Toxic 
Oligomers Formed in Vitro from Mammalian-derived Expanded huntingtin Exon-1 Protein, Journal of Biological Chemistry 287, 16017-16028.

(14) Burke, K. A., Kauffman, K. J., Umbaugh, C. S., Frey, S. L., and Legleiter, J. (2013) The Interaction of Polyglutamine Peptides With Lipid Membranes is Regulated by Flanking Sequences Associated with Huntingtin, Journal of Biological Chemistry.

(15) Burke, K. A., Hensal, K. M., Umbaugh, C. S., Chaibva, M., and Legleiter, J. (2013) Huntingtin disrupts lipid bilayers in a polyQ-length dependent manner, Biochimica et Biophysica Acta (BBA) - Biomembranes 1828, 1953-1961.

(16) Tam, S., Spiess, C., Auyeung, W., Joachimiak, L., Chen, B., Poirier, M. A., and Frydman, J. (2009) The chaperonin TRiC blocks a huntingtin sequence element that promotes the conformational switch to aggregation, Nature Structural \& Molecular Biology 16, 1279-U1298.

(17) Bugg, C. W., Isas, J. M., Fischer, T., Patterson, P. H., and Langen, R. (2012) Structural Features and Domain Organization of Huntingtin Fibrils, Journal of Biological Chemistry 287, 31739-31746.

(18) Jayaraman, M., Mishra, R., Kodali, R., Thakur, A. K., Koharudin, L. M. I., Gronenborn, A. M., and Wetzel, R. (2012) Kinetically Competing Huntingtin Aggregation Pathways Control Amyloid Polymorphism and Properties, Biochemistry 51, 2706-2716.

(19) Legleiter, J., Mitchell, E., Lotz, G. P., Sapp, E., Ng, C., DiFiglia, M., Thompson, L. M., and Muchowski, P. J. (2010) Mutant Huntingtin Fragments Form Oligomers in a Polyglutamine Length-dependent Manner in Vitro and in Vivo, Journal of Biological Chemistry 285, 14777-14790.

(20) Williamson, T. E., Vitalis, A., Crick, S. L., and Pappu, R. V. (2010) Modulation of Polyglutamine Conformations and Dimer Formation by the N-Terminus of Huntingtin, Journal of Molecular Biology 396, 1295-1309.

(21) Mishra, R., Hoop, C. L., Kodali, R., Sahoo, B., van der Wel, P. C. A., and Wetzel, R. (2012) Serine Phosphorylation Suppresses Huntingtin Amyloid Accumulation by Altering Protein Aggregation Properties, Journal of Molecular Biology 424, 114.

(22) Gu, X. F., Greiner, E. R., Mishra, R., Kodali, R., Osmand, A., Finkbeiner, S., Steffan, J. S., Thompson, L. M., Wetzel, R., and Yang, X. W. (2009) Serines 13 and 16 Are Critical Determinants of Full-Length Human Mutant Huntingtin Induced Disease Pathogenesis in HD Mice, Neuron 64, 828-840.

(23) Atwal, R. S., Desmond, C. R., Caron, N., Maiuri, T., Xia, J. R., Sipione, S., and Truant, R. (2011) Kinase inhibitors modulate huntingtin cell localization and toxicity, Nature Chemical Biology 7, 453-460.

(24) Shirasaki, D. I., Greiner, E. R., Al-Ramahi, I., Gray, M., Boontheung, P., Geschwind, D. H., Botas, J., Coppola, G., Horvath, S., Loo, J. A., and Yang, X. W. (2012) Network Organization of the Huntingtin Proteomic Interactome in Mammalian Brain, Neuron 75, 41-57.

(25) Kar, K., Hoop, C. L., Drombosky, K. W., Baker, M. A., Kodali, R., Arduini, I., van der Wel, P. C. A., Horne, W. S., and Wetzelt, R. (2013) beta-Hairpin-Mediated Nucleation of Polyglutamine Amyloid Formation, Journal of Molecular Biology 425, 1183-1197.

2. Lysine Residues in the N-Terminal Huntingtin Amphipathic $\alpha$-Helix Play a Key Role in Peptide Aggregation 
(26) Schneider, R., Schumacher, M. C., Mueller, H., Nand, D., Klaukien, V., Heise, H., Riedel, D., Wolf, G., Behrmann, E., Raunser, S., Seidel, R., Engelhard, M., and Baldus, M. (2011) Structural Characterization of Polyglutamine Fibrils by SolidState NMR Spectroscopy, Journal of Molecular Biology 412, 121-136.

(27) Thakur, A. K., Jayaraman, M., Mishra, R., Thakur, M., Chellgren, V. M., Byeon, I. J. L., Anjum, D. H., Kodali, R., Creamer, T. P., Conway, J. F., Gronenborn, A. M., and Wetzel, R. (2009) Polyglutamine disruption of the huntingtin exon $1 \mathrm{~N}$ terminus triggers a complex aggregation mechanism, Nature Structural \& Molecular Biology 16, 380-389.

(28) Olshina, M. A., Angley, L. M., Ramdzan, Y. M., Tang, J. W., Bailey, M. F., Hill, A. F., and Hatters, D. M. (2010) Tracking Mutant Huntingtin Aggregation Kinetics in Cells Reveals Three Major Populations That Include an Invariant Oligomer Pool, Journal of Biological Chemistry 285, 21807-21816.

(29) Sahoo, B., Singer, D., Kodali, R., Zuchner, T., and Wetzel, R. (2014) Aggregation Behavior of Chemically Synthesized, Full-Length Huntingtin Exon1, Biochemistry 53, 3897-3907.

(30) Chen, S. M., and Wetzel, R. (2001) Solubilization and disaggregation of polyglutamine peptides, Protein Science 10, 887-891.

(31) Burke, K. A., Godbey, J., and Legleiter, J. (2011) Assessing mutant huntingtin fragment and polyglutamine aggregation by atomic force microscopy, Methods 53, 275-284.

(32) Rand, K. D., Lund, F. W., Amon, S., and Jorgensen, T. J. D. (2011) Investigation of amide hydrogen back-exchange in Asp and His repeats measured by hydrogen $(\mathrm{H}-1 / \mathrm{H}-2)$ exchange mass spectrometry, International Journal of Mass Spectrometry 302, 110-115.

(33) Srinivasan, R., Jones, E. M., Liu, K., Ghiso, J., Marchant, R. E., and Zagorski, M. G. (2003) pH-Dependent Amyloid and Protofibril Formation by the ABri Peptide of Familial British Dementia, Journal of Molecular Biology 333, 1003-1023.

(34) Groenning, M., Norrman, M., Flink, J. M., van de Weert, M., Bukrinsky, J. T., Schluckebier, G., and Frokjaer, S. (2007) Binding mode of Thioflavin T in insulin amyloid fibrils, Journal of Structural Biology 159, 483-497.

(35) Vitalis, A., and Pappu, R. V. (2011) Assessing the contribution of heterogeneous distributions of oligomers to aggregation mechanisms of polyglutamine peptides, Biophysical Chemistry 159, 14-23.

(36) Kheterpal, I., Cook, K. D., and Wetzel, R. (2006) Hydrogen/Deuterium Exchange Mass Spectrometry Analysis of Protein Aggregates, In Methods in Enzymology (Indu, K., and Ronald, W., Eds.), pp 140-166, Academic Press.

(37) Pitteri, S. J., Chrisman, P. A., Hogan, J. M., and McLuckey, S. A. (2005) Electron transfer ion/ion reactions in a three-dimensional quadrupole ion trap: Reactions of doubly and triply protonated peptides with SO2 center dot, Analytical Chemistry 77, 1831-1839.

(38) McLuckey, S. A., and Stephenson, J. L. (1998) Ion ion chemistry of high-mass multiply charged ions, Mass Spectrometry Reviews 17, 369-407.

(39) Syka, J. E. P., Coon, J. J., Schroeder, M. J., Shabanowitz, J., and Hunt, D. F. (2004) Peptide and protein sequence analysis by electron transfer dissociation 
mass spectrometry, Proceedings of the National Academy of Sciences of the United States of America 101, 9528-9533.

(40) Rand, K. D., Pringle, S. D., Morris, M., and Brown, J. M. (2012) Site-Specific Analysis of Gas-Phase Hydrogen/Deuterium Exchange of Peptides and Proteins by Electron Transfer Dissociation, Analytical Chemistry 84, 1931-1940.

(41) Pan, J. X., Han, J., Borchers, C. H., and Konermann, L. (2012) Structure and Dynamics of Small Soluble A beta(1-40) Oligomers Studied by Top-Down Hydrogen Exchange Mass Spectrometry, Biochemistry 51, 3694-3703.

(42) Perutz, M. F., Johnson, T., Suzuki, M., and Finch, J. T. (1994) GLUTAMINE REPEATS AS POLAR ZIPPERS - THEIR POSSIBLE ROLE IN INHERITED NEURODEGENERATIVE DISEASES, Proceedings of the National Academy of Sciences of the United States of America 91, 5355-5358.

(43) Nelson, R., Sawaya, M. R., Balbirnie, M., Madsen, A. O., Riekel, C., Grothe, R., and Eisenberg, D. (2005) Structure of the cross-beta spine of amyloid-like fibrils, Nature 435, 773-778.

(44) Rand, K. D., Lund, F. W., Amon, S., and Jørgensen, T. J. D. (2011) Investigation of amide hydrogen back-exchange in Asp and His repeats measured by hydrogen $(1 \mathrm{H} / 2 \mathrm{H})$ exchange mass spectrometry, International Journal of Mass Spectrometry 302, 110-115.

(45) Michalek, M., Salnikov, E. S., and Bechinger, B. (2013) Structure and Topology of the Huntingtin 117 Membrane Anchor by†a†Combined Solution and SolidState NMR Approach, Biophysical journal 105, 699-710.

(46) Atwal, R. S., and Truant, R. (2008) A stress sensitive ER membrane-association domain in Huntingtin protein defines a potential role for Huntingtin in the regulation of autophagy, Autophagy 4, 91-93.

(47) Nagarajan, A., Jawahery, S., and Matysiak, S. (2013) The Effects of Flanking Sequences in the Interaction of Polyglutamine Peptides with a Membrane Bilayer, The Journal of Physical Chemistry B. 


\section{Huntingtin $\mathrm{N}$-terminal monomeric and multimeric structures}

\section{destabilized by covalent modification of heteroatomic residues ${ }^{1}$}

${ }^{1}$ Reproduced with permission from Biochemistry, submitted for publication. Arndt, J. R., Kondalaji S. G., Maurer, M. M., Parker A., Legleiter J., Valentine S.J. submitted to Biochemistry. Unpublished work copyright 2015 American Chemical Society.

Early-stage oligomer formation of the huntingtin protein is driven by selfassociation of the seventeen-residue amphipathic $\alpha$-helix at the protein's $\mathrm{N}$ terminus (Nt17). These oligomeric structures have been implicated in neuronal toxicity and may represent the most neurotoxic species in Huntington's disease. Therefore, a residue-specific structural characterization of $\mathrm{Nt} 17$ is crucial to understanding and potentially inhibiting oligomer formation. Native electrospray ion mobility spectrometry-mass spectrometry (IMS-MS) techniques and molecular dynamics simulations (MDS), have been applied to study coexisting monomer and multimer conformations of $\mathrm{Nt17}$, independent of the remainder of huntingtin exon 1. From the MDS experiments for the dimer ions, $\mathrm{K} 6$ residues in both monomer constituents interact with $\mathrm{S} 16$ and E12 residues on adjacent peptides. Decrease in multimer formation as a function of covalent modification could result from disruption of this interaction. This work provides critical insight toward Nt17 multimerization and possibly, the early stages of huntingtin exon 1 aggregation

3. Huntingtin N-terminal monomeric and multimeric structures destabilized by 


\subsection{Introduction: Nt17 interactions and modifications studied by IMS-MS}

Huntington's disease is a fatal neurodegenerative disease caused by an expanded, glutamine-coding CAG repeat sequence in the huntingtin gene ${ }^{1}$. The resulting expanded (>37 residue) polyglutamine (polyGln) tract in huntingtin exon 1 is responsible for aggregation ${ }^{2,3}$. Directly adjacent (N-terminal) to the polyGln tract is a seventeen-residue amphipathic $\alpha$-helix (Nt17). This tract is the driving force behind potentially toxic oligomer formation ${ }^{4,5}$, anchoring to a lipid substrate ${ }^{6-8}$, and cellular trafficking ${ }^{9}, \mathrm{Nt17}$ association is the critical first step in oligomerization ${ }^{5}$. In the present work Nt17, independent of the polyGIn domain, was used to model the earliest stages of Nt17-driven oligomer formation of the huntingtin protein. A motivating factor in this study is that modifications of this sequence at selected side-chains may be used to glean insight to inhibiting toxic oligomerization. To date, no study has directly probed interactions of Nt17 monomers or downstream effects of monomer modification on multimer formation and structure. This study serves as a model for early stages of Nt17mediated huntingtin aggregate nucleation and provides insight into key residues in initial Nt17 association.

Nt17 populates multiple secondary structures, ranging from $75 \%$ randomcoil to mostly helical, depending on the buffer and proximity of binding partners 10-12. Jayaraman et al. suggest that $\mathrm{Nt17}$ adopts a helical structure only upon interaction with a second $\mathrm{Nt} 17$ tract ${ }^{5}$. Nt17 maintains its helical structure in fibrillar forms of huntingtin exon $1^{13}$, which suggests the helical conformation is relatively stable within the fibril. Post-translational modifications of $\mathrm{Nt17}$, such as

3. Huntingtin $N$-terminal monomeric and multimeric structures destabilized by 
phosphorylation of T3, S13 and S16, retard Nt17-mediated aggregation possibly by stabilizing a random coil arrangement and thus preventing formation of helical structure ${ }^{14-16}$.

The current study demonstrates the inherent structural heterogeneity of a sequence that is implicated in potentially toxic oligomer formation in Huntington's disease. When combined with molecular dynamics simulations (MDS), two monomeric structural types resembling partial helices (proposed solution structures) are observed. Additionally, several multimeric species are observed, ranging from compact globular to extended helical bundle. For dimer ions, MDS suggests that the most elongated, and potentially most relevant, conformation is an arrangement of antiparallel helices stabilized by intermolecular hydrogen bonding interactions. Finally, covalent modification reveals solvent accessibility of residues near the $\mathrm{N}$-terminus of the peptide at the boundary of the hydrophobic and hydrophilic faces. This modification serves to destabilize multimer formation. This is the first instance of direct, structurally-specific, monitoring of Nt17 oligomer formation. This model provides insights into the structural underpinnings of Nt17-driven huntingtin oligomerization while also providing a potential target for inhibition.

\subsection{Materials and Methods}

\subsubsection{Sample Preparation.}

Nt17 peptide was purchased as a lyophilized powder (Genscript, Inc.). 1.0 mg of powder was dissolved in $1.0 \mathrm{~mL}$ of $50 / 50$ trifluoroacetic

3. Huntingtin N-terminal monomeric and multimeric structures destabilized by 
acid/hexafluoroisopropanol (TFA/HFIP) and separated into $200 \mu \mathrm{g}$ aliquots. All solvent was evaporated by a stream of nitrogen, then samples were placed in a vacuum evaporator for three hours to create a peptide film. Films were stored at $20^{\circ} \mathrm{C}$ until use. All samples were reconstituted in $0.1 \mathrm{M}$ ammonium acetate (Fisher) to yield a final concentration of $0.2 \mathrm{mg} \mathrm{mL}^{-1}$. All water used was purified to $18.2 \mathrm{M} \Omega$ resistance (Millipore).

\subsubsection{Carbethoxylation with diethylpyrocarbonate (DEPC).}

DEPC solutions were made by diluting stock DEPC (Thermo) into LC-MS grade acetonitrile (Fisher). Typically, structural analyses avoid the use of organics as much as possible; however, DEPC has a very low solubility in water, so organic solvents, in this case, were unavoidable. Freshly reconstituted samples were labeled at $5 \times, 50 \times$, and $250 \times($ mol DEPC:mol Nt17) concentrations. In each case, $1 \%$ or less $(\mathrm{v} / \mathrm{v})$ acetonitrile was added to the samples. Samples were allowed to react for 10 minutes before a $200 \mu \mathrm{L}$ aliquot was removed for electrospray.

\subsubsection{Ion Mobility Spectrometry-Linear Ion Trap Mass Spectrometry (IMS-MS).}

Samples were analyzed on a hybrid (home-built) IMS-linear ion trap (LIT) mass spectrometer (Thermo), which has been described in detail elsewhere ${ }^{55,56}$, as well as in Chapter 1. The ESI voltage was maintained at $1.7 \mathrm{kV}$, with a $0.5 \mu \mathrm{L}$ $\mathrm{min}^{-1}$ solution flow rate. ESI emitters were pulled from untreated fused silica capillary. A linear voltage drop was applied along the length of the drift tube to

3. Huntingtin N-terminal monomeric and multimeric structures destabilized by 
give a constant field of $1000 \mathrm{~V} \mathrm{~m}^{-1}$. Ions are trapped at the front of the drift tube after electrospray by an ion funnel ${ }^{57}$, where they are introduced at $20.0 \mathrm{~ms}$ intervals. The potential on a second gate located near the drift tube exit was lowered to allow ions into the mass spectrometer (Thermo LTQ Velos Pro, Thermo Scientific, San Jose, CA). The delay between the front gate and the back gate is scanned to create the IMS-MS spectrum. Each mass spectrum was collected for 30 seconds at each delay time setting, with a 400 ms maximum ion trap injection time. Four microscans were averaged to generate the mobilityresolved mass spectra. All mass spectra were collected from 150 to $2000 \mathrm{~m} / \mathrm{z}$.

\subsubsection{Molecular dynamics.}

MDS experiments required the construction of the $[\mathrm{M}+2 \mathrm{H}]^{2+}$ monomer and $[\mathrm{M}+3 \mathrm{H}]^{3+}$ dimer peptide ions. The charge sites for these ions were selected from experimental mass spectra. The $\varepsilon$-nitrogens of residues $\mathrm{K} 9$ and $\mathrm{K} 15$ were protonated for the doubly-charged monomer, while both $\mathrm{K} 9$ residues and a single K15 residue were charged for the triply-charged dimer ion. Although a tentative selection, these residues are chosen as the charge site locations because upon carbethoxylation of $\mathrm{K} 6$, no reduction in $[\mathrm{M}+2 \mathrm{H}]^{2+}$ ion production is observed (see Results and Discussion). One linear starting structure was employed for each charge state. Each initial structure was optimized at a quantum chemical level of $\mathrm{HF} / 6-31 \mathrm{G}(\mathrm{d})$ and used for calculating molecular electrostatic potential (MEP) values and charge fitting performed with the R.E.D. Server ${ }^{58-61}$. To generate the initial extended structures for MDS, the AMBER force field FF12SB was

3. Huntingtin N-terminal monomeric and multimeric structures destabilized by covalent modification of heteroatomic residues 
employed. The method used for annealing was similar to that described previously ${ }^{62}$. Energy-minimized conformers were subjected to simulated annealing for 40 ps using the AMBER12 package ${ }^{63}$. First the conformers were subjected to dynamics at $1000 \mathrm{~K}$. Next, they were subjected to a gradual temperature gradient (to $50 \mathrm{~K}$ ) and were subsequently energy-minimized. For each ion conformer study, 1000 cycles of annealing were conducted for 40 ps and the final, energy-minimized structure from one cycle was subjected to dynamics at $1000 \mathrm{~K}$ for the next cycle. The 1000 cycles of annealing were conducted with no non-bonded cutoffs to generate 1000 candidate structures. The collision cross sections of the 1000 conformers were calculated using the Mobcal ${ }^{64}$ software employing the trajectory method $(\mathrm{TM})^{65}$. The potential energies of the 1000 conformers were plotted as a function of collision cross section. Matching ( $<1 \%$ difference in collision cross section), low-energy ion structures were selected for comparisons to experimental data. Structures were illustrated using VMD ver. 1.9.2.

\subsection{Results and Discussion}

\subsubsection{Nt17 Adopts Several Monomeric and Multimeric Conformations.}

IMS-MS distributions produced upon electrospray of the unlabeled sample are shown in Figure 3.1. Multimers up to, and including, a tetramer are observed. The mass spectrum is dominated by the doubly-charged monomer $\left([\mathrm{M}+2 \mathrm{H}]^{2+}\right)$ ions, which primarily adopt two conformations that are mobility resolved (Figure 3.1B). The first conformer, arriving at $11.0 \mathrm{~ms}$, is of higher abundance ( 4 fold) 

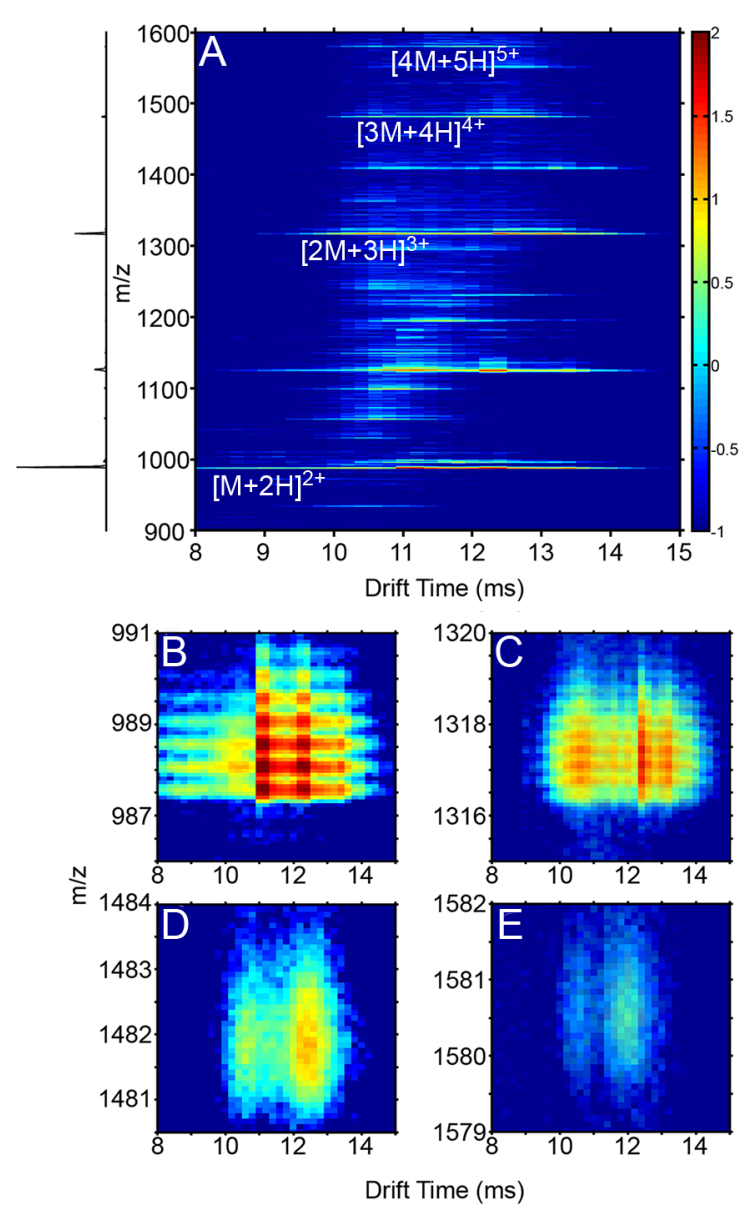

Figure 3.1. False color IMS-MS distributions of Nt17 ions. Colored intensity is presented in log scale. All panels are represented with the same color scale. a.) Full IMS-MS scan with associated mass spectrum (left). b., c., d., and e.) Enhanced images of monomer, dimer, trimer, and tetramer structures, respectively. than the other as demonstrated in the drift time distributions shown in Figure 3.3A. The second, less-intense, feature corresponding to doubly-charged monomer ions exhibits a longer drift time ( 12.4 ms). From Equation 1, collision cross sections for the compact and more elongated $[\mathrm{M}+2 \mathrm{H}]^{2+}$ ions are determined to be $399.8 \pm 0.8$ and $442.7 \pm 0.1 \AA^{2}$ (see Table 1$)$.

At least four distinct

dataset features corresponding to triply-charged dimer $\left([2 \mathrm{M}+3 \mathrm{H}]^{3+}\right)$ ions are observed in the two-dimensional (2D) IMS-MS datasets (Figure 3.1C). A broad feature $\left(t_{D} \sim 1\right.$ ms FWHM) is observed at a drift time of $\sim 10.6 \mathrm{~ms}$ as shown in the $2 \mathrm{D}$ drift time distribution in Figures 3.1B - E, as well as the $t_{D}$ distribution in Figure 3.3. Narrower features are observed at $t_{D} 11.6$ and $12.7 \mathrm{~ms}$ (Figure 3.3A). The dataset feature at 12.7 represents the most intense peak for the $[2 \mathrm{M}+3 \mathrm{H}]^{3+}$ ions,

3. Huntingtin N-terminal monomeric and multimeric structures destabilized by 
exhibiting a peak intensity that is $\sim 2$ fold greater than the other dataset features, which demonstrate similar intensity levels. A separate dataset feature representing a shoulder on the most intense peak is observed at a $t_{D}$ of $13.2 \mathrm{~ms}$.

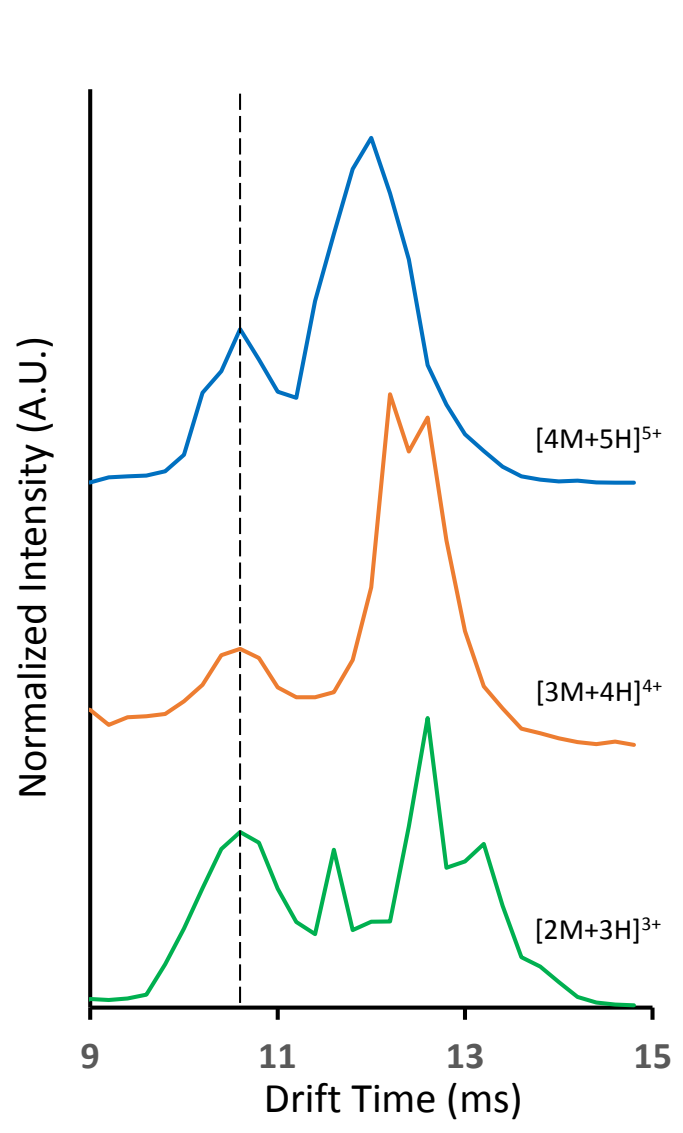

Figure 3.2 - Extracted $t_{D}$ distributions for dimer (green), trimer (orange), and tetramer (bllue) ions. The dotted box shows the feature at $10.6 \mathrm{~ms}$ for all ions, suggesting that this feature arises from dissociation of a higher-order complex(es) after the drift separation.
The collision cross sections for the partially-resolved $[2 \mathrm{M}+3 \mathrm{H}]^{3+}$ ions are $568.1 \pm 0.3,620.3 \pm 0.1,671 \pm 5$, and $708 \pm 1 \AA^{2}$ for the various conformations in order of increasing size. The broad feature at 10.6 ms appears in essentially all multimeric $t_{D}$ distributions, up to and including the tetramer (Figure 3.2).

In each spectrum, the feature has the same peak shape. This may suggest the feature is derived from higher-order $(n>4)$ multimer species that dissociate after the drift separation. In support of this argument, a calculated collision cross-section for a triply-charged dimer ion occurring at $10.6 \mathrm{~ms}$ is $568 \AA^{2}$. This value is $\sim 10 \%$ smaller than all of the smallest dimer configurations sampled by MDS (see 3.3.3 Conformations of 

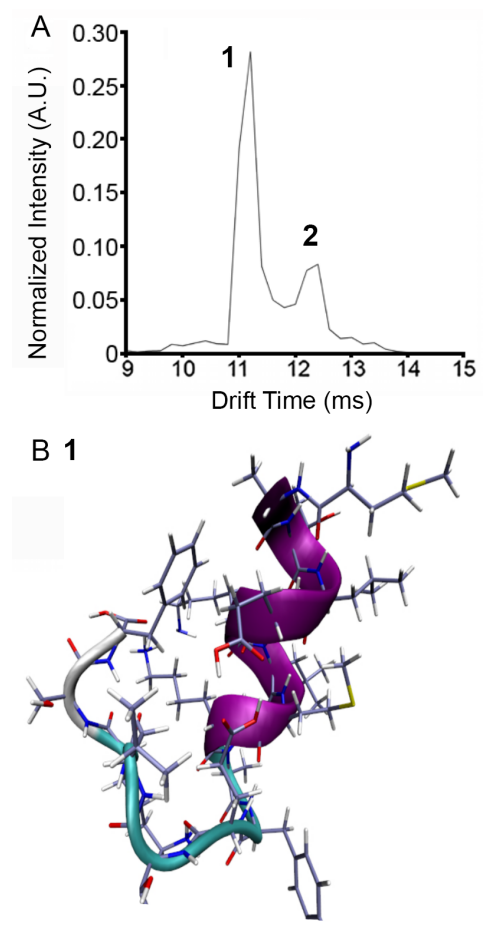

C 2

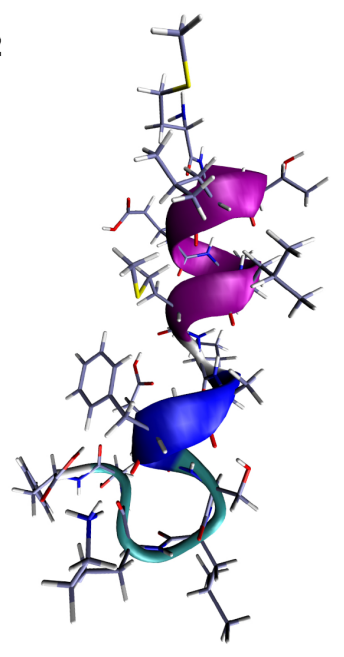

Figure 3.3. Nt17 ribbon structures calculated from MDS. Pink regions are $\alpha$ helical. Blue regions correspond to a 3-10 helix. a.) Extracted $t_{D}$ distribution for $[\mathrm{M}+2 \mathrm{H}]^{2+}$ ions. b.) Compact conformer (1) at $11.0 \mathrm{~ms}$. c.) Extended conformer (2) at $12.4 \mathrm{~ms}$. See Table 1 for assigned collision cross-sections
Multimeric lons results section) and matches closely ( $2.3 \%$ larger) to a spherical compact arrangement ${ }^{45}$. Early-stage oligomers would be expected to consist of arrangements of elongated helices, as observed in fibril structures 5, 13,66. Unfortunately, the multimer from which the 10.6 ms feature may have arisen is not distinguished in the 2D distribution and cannot be reliably identified.

Several higher-order multimeric structures $\left([3 \mathrm{M}+4 \mathrm{H}]^{4+}\right.$ and $[4 \mathrm{M}+5 \mathrm{H}]^{5+}$ ions, Figures $1 \mathrm{D}$ and E) are also evident in the $2 \mathrm{D}$ spectrum. For the former ions, the same broad dataset feature centered at a drift time of 10.6 ms was observed as in the other conformations. A narrower conformer with a collision cross section of 886 $\AA^{2}$ is observed at a longer drift time $(12.4 \mathrm{~ms})$. For the $[4 \mathrm{M}+5 \mathrm{H}]^{5+}$ ions, a broad dataset feature is observed to have collision cross-section of $1073 \AA^{2}$.

\section{Huntingtin N-terminal monomeric and multimeric structures destabilized by}




\subsubsection{Compact and elongated $\mathrm{Nt17}[\mathrm{M}+2 \mathrm{H}]^{2+}$ Ion Structures from Molecular}

\section{Dynamics Simulations.}

Solution studies have proposed that $\mathrm{Nt17}$ exists primarily as a random coil in a native-like environment ${ }^{13}$. Separate studies, both solution ${ }^{67,68}$ and simulation ${ }^{12,69,70}$, have suggested the presence of helical monomers in solution. The IMS data presented here (Figure 3.1 and Figure 3.3) suggests the presence of two dominant coexisting solution structures for peptide monomers.

Figure 3.3A shows the $t_{D}$ distribution of the doubly-charged monomer. Figure 3.3B and C depict energy-minimized compact (Structure 1) and elongated (Structure 2) structures of Nt17 obtained from MDS. These structures represent the lowest energy conformers that are within $1 \%$ of the experimental collision cross-sections of the two $[\mathrm{M}+2 \mathrm{H}]^{2+}$ dataset features. It should be noted here, that all structures presented in the present study represent gas-phase conformations that best fit to experimental gas phase conformers arising from different solution structures ${ }^{30}$. That is, although the monomer structures are modeled in the gasphase, they may not completely resemble solution conformers.

Both monomeric conformers are mostly helical. The high degree of helical propensity for both structures is consistent with proposed and experimentally observed solution structures contained within a huntingtin peptide aggregate ${ }^{13,}$ 69, 71 . The primary difference between the compact and elongated monomer structures is the propensity for the random coil region (residues 11 to 17 ) in Structure 1 to turn back on itself and potentially associate with the helix (Figure 3.3B). In the elongated structure, the portion C-terminal to the helical region is 
extended away from the rest of the peptide. Additionally, Structure 2 contains a 3-10 helix spanning residues A10 to E12. This helix could prohibit C-terminal association as is observed in Structure 1. In the Structure 1, residue K6 is in relative proximity to $\mathbf{S} 16$. Interestingly, $\mathrm{K} 6$ is potentially involved in intermolecular interaction from an early aggregate state ${ }^{52}$. In solution, such proximity could lead to charge-dipole interactions between the $\varepsilon$-amino group on $\mathrm{K} 6$ and the primary alcohol group on S16. Previous experiments have shown that phosphorylation and S16D mutations decrease aggregation kinetics through inhibition of Nt17driven oligomerization ${ }^{14,15}$. One hypothesis for the inhibition of oligomer formation could be a charge-charge interaction formed between $\mathrm{K} 6$ and residue $\mathrm{S} 16$, though the proximity could be due to charge solvation of the neighboring $\mathrm{K} 9$ in the gas phase. Still, if this is reminiscent of a solution structure, this interaction could prevent the formation of the extended helices observed in short huntingtin peptide oligomers and mature huntingtin fibrils ${ }^{5,13}$. It is intriguing that both of these structures match closely to the extended solution conformer found by Kelley et al ${ }^{71}$. Indeed, the calculated collision-cross section of the elongated structure modeled here is within $\pm 3 \%$ of the proposed solution helix.

\subsubsection{Conformations of Multimeric lons.}

Simulated annealing was performed on a dimeric species to gain some insight to possible gas-phase structures that arise from solution conformations. Figure $3.4 \mathrm{~A}$ shows the $t_{D}$ distribution of all dimer species. Figure $3.4 \mathrm{~B}, \mathrm{C}$, and D depict the results from the simulated annealing experiment (Structures 3, 4, and

3. Huntingtin $N$-terminal monomeric and multimeric structures destabilized by 
5 , respectively). Structure 3 corresponds to a dimer ion species that would have a $t_{D}$ of $11.2 \mathrm{~ms}\left(622 \AA^{2}\right)$, while Structures 4 and 5 depict ions that would have a $t_{D}$ of $12.6\left(666.3 \AA^{2}\right)$ and $13.2 \mathrm{~ms}\left(709.9 \AA^{2}\right)$, respectively. Structure 3 is composed of two partial 3-10 helices that are arranged skew to one another, which could

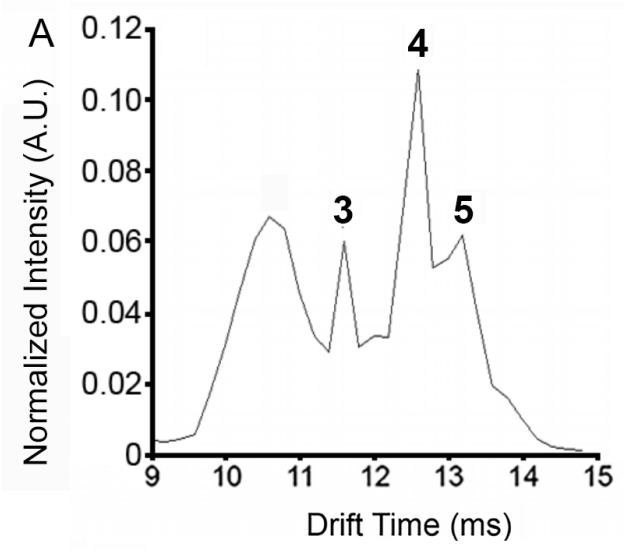

C 4
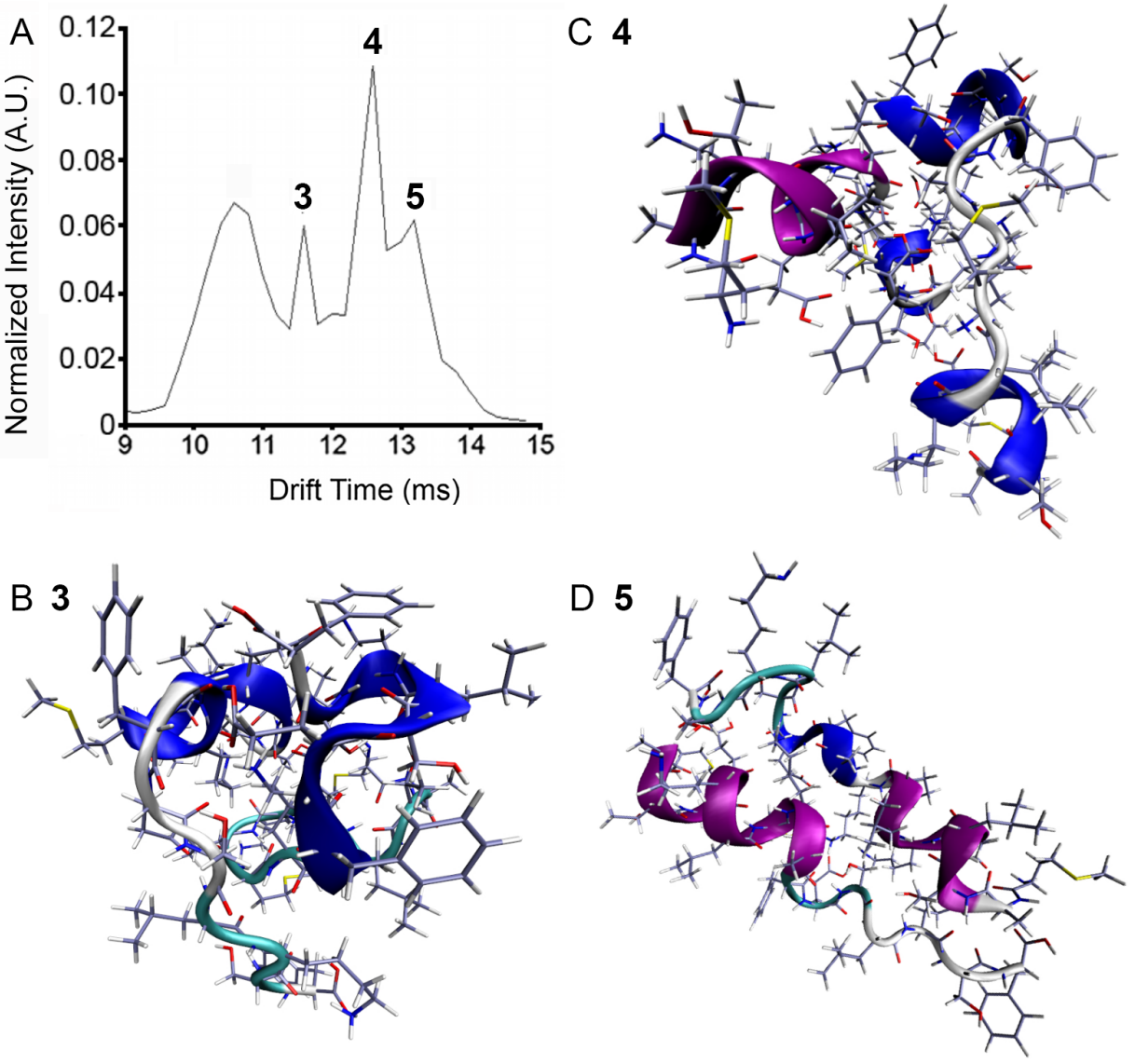

Figure 3.4. Nt17 dimer structures calculated from MDS. Color schemes are the same as in Figure 3.3 a.) Extracted arrival time distribution. '3', '4', and '5' correspond to relevant dimer structures, depicted in panels b., c., and d., respectively.

represent the first step in transition to structures representative of Structures 4 and 5. The next, more elongated, conformer, Structure 4, consists of two partially helical monomers that are arranged offset to one another. The a-helix in one monomer ranges from the $\mathrm{N}$-terminus to $\mathrm{A} 10$, where a random coil region leads

3. Huntingtin N-terminal monomeric and multimeric structures destabilized by 
into a 3-10 helix. This monomer constituent resembles Structure 2. The second monomer exhibits two 3-10 helices: one from the $\mathrm{N}$-terminus to $\mathrm{E} 5$, the other from $\mathrm{K} 15$ to $\mathrm{F} 17$. It should be stressed here that the structures obtained from simulation are gas-phase structures and the actual degree of similarity to solution structures is unknown. Still, reliable estimates of solution conformation have been presented from gas-phase structures ${ }^{27,30,31}$. It is also noteworthy that elements of secondary structure similar to those found in the monomer species (and contained in a fibril ${ }^{5,13}$ ) are observed for the dimers.

Structure 5 depicts a structure obtained from MDS that is consistent with the most elongated conformation of the Nt17 dimer. In this model, both peptides are partially helical, with the $\alpha$-helix spanning from the $\mathrm{N}$-terminus to $\mathrm{A} 10$ in one monomer, and an extended a-helix-coil-3-10 helix motif in the second monomer. Interestingly, the helices are arranged antiparallel to one another. K6 interactions stabilize the dimer structure through hydrogen bonding; at one end, $\mathrm{K} 6$ associates with $\mathrm{S} 16$, while at the other end $\mathrm{K} 6$ interacts with E12. Previous solution docking simulations have alluded to a stabilizing K6-S16 interaction in antiparallel Nt17 helices ${ }^{10}$. Additionally, solution-based deuterium exchange measurements revealed an increased role of K6 in Nt17 multimer structure ${ }^{52}$. Finally, S13D and S16D mutations in Nt17 prevented aggregation of huntingtin exon 1 synthetic models via the Nt17-mediated pathway ${ }^{14}$. The dimer conformations resemble the assembly of two elongated monomer structures (Structure 5). Considering structure comparisons above, as well as the native 
ESI conditions, these analyses may well provide information regarding Nt17 binding face in the earliest stages of oligomer formation.

For the $[3 \mathrm{M}+4 \mathrm{H}]^{4+}$ ions, the larger ions (Figure 3.1D) with collision cross sections of $873 \AA^{2}$ and $902 \AA^{2}$ are $\sim 22 \%$ and $24 \%$ larger, respectively, than expected values for globular structures based on a fit to experimental data ${ }^{45}$. The larger ions show a percent difference that is near that ( $21 \%)$ expected for helical bundles of this size but well below that $(\sim 51 \%)$ determined for helices joined end on. The calculated cross section of the elongated trimer is near that of insulin A chain packed as a helical bundle ( $874 \AA^{2}$ vs. $840 \AA^{2}$ for insulin A chain) ${ }^{45}$. The two peptides are similar in length, so similarity in gas-phase helical packing would be expected. The $[4 \mathrm{M}+5 \mathrm{H}]^{5+}$ ion conformers (Figure 3.1E) display a similar trend. The elongated $\left(1073 \AA^{2}\right)$ ion has a collision cross section that is $\sim 26 \%$ larger than the expected value for a globular multimeric structure based on a fit to experimental data. This value is slightly above the percent difference for helical bundles $(\sim 16)$, and well below that expected for the percent difference increase ( $75 \%)$ for helices arranged end on. Additoinally, the calculated cross section of the Nt17 tetramer, $1073 \AA^{2}$, is in the same range as helical bundled insulin A chain (approximately $1010 \AA^{2}$ ) The lack of a polyGIn domain in the current study should be considered. In a fibril, polyGln $\beta$-sheet structure can impinge upon the Nt17 region at the last two Nt17 residues ${ }^{17}$. In the present study, the C-terminal portion of Nt17 are disordered, so structural transition to $\beta$ sheet is a possibility.

3. Huntingtin N-terminal monomeric and multimeric structures destabilized by 

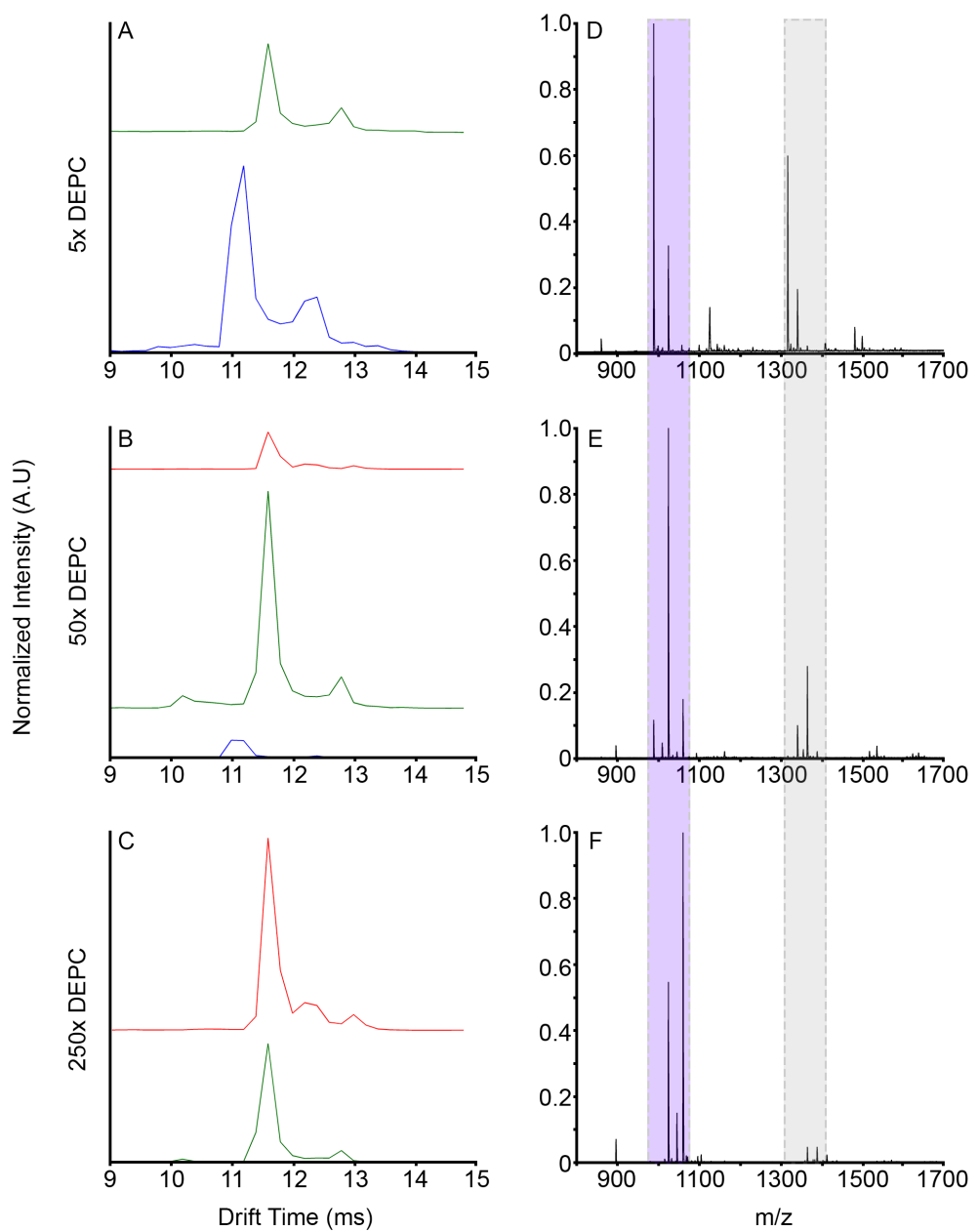

Figure 3.5. $t_{D}$ distributions of covalently modified $\mathrm{Nt17}[\mathrm{M}+2 \mathrm{H}]^{2+}$ monomer at $5 x$ (A), 50x (B), and 250x (C) DEPC. $t_{D}$ distributions are normalized to total peptide ion counts. Blue trace: unlabeled Nt17; green trace: singly modified Nt17; red trace: doubly modified. $D-F$, mass spectra at each DEPC concentration showing monomer $\mathrm{m} / \mathrm{z}$ range (violet box) and dimer $\mathrm{m} / \mathrm{z}$ range (gray box).

Covalent modification with DEPC was performed to determine effects of charged side chain modification on Nt17 multimerization. Figure 3.5 shows IMS-MS distributions of $[\mathrm{M}+2 \mathrm{H}]^{2+}$ peptide ions at various states of modification after ten

3. Huntingtin N-terminal monomeric and multimeric structures destabilized by 
minutes of incubation with DEPC (for 2D IMS-MS distributions depicting the course of modification, see Figures 3.6 and 3.7). Drift times and collision cross sections for each species are presented in Table 1.

\begin{tabular}{|c|c|c|c|}
\hline Species $^{a}$ & $\mathrm{~m} / \mathrm{z}^{b}$ & $t_{d}(m s)^{c}$ & $\Omega\left(\AA^{2}\right)^{d}$ \\
\hline \multirow[t]{2}{*}{$\mathrm{Nt17}$} & 988.1 & 11.2 & $400 \pm 1$ \\
\hline & & 12.4 & $443 \pm 1$ \\
\hline \multirow[t]{3}{*}{$\mathrm{Nt} 17 \times 2$} & 1317 & 11.6 & $620 \pm 1$ \\
\hline & & 12.6 & $671 \pm 5$ \\
\hline & & 13.2 & $708 \pm 1$ \\
\hline $\mathrm{Nt} 17 \times 3$ & 1481 & 12.6 & $874 \pm 1$ \\
\hline $\mathrm{Nt} 17 \times 4$ & 1581 & 12.0 & $1074 \pm 1$ \\
\hline \multirow[t]{2}{*}{ Nt17 N/T3C } & 1024 & 11.6 & $413 \pm 0$ \\
\hline & & 12.8 & $457 \pm 1$ \\
\hline \multirow[t]{4}{*}{ Nt17 T3C, K6C } & 1060 & 11.6 & $414 \pm 1$ \\
\hline & & 12.2 & $436 \pm 1$ \\
\hline & & 13.0 & $465 \pm 1$ \\
\hline & & 13.6 & $729 \pm 1$ \\
\hline Nt17 x 2 N/T3C, K6C* & 1389 & 13.4 & $718 \pm 1$ \\
\hline
\end{tabular}

Table 1. Calculated collision cross-sections for selected Nt17 modified and unmodified conformers. ${ }^{a}$ Species are identified as monomer (Nt17) with carbethoxylated residue identified. *Two monomer units were modified near the $N$-terminus, one was modified at K6. ${ }^{b}$ measured $\mathrm{m} / \mathrm{z}$ value. ${ }^{c}$ measured drift time in $\mathrm{ms} .{ }^{d}$ calculated collision cross section with standard deviation ( $n=3$ replicates).

At $5 \times$ label (Figure 3.5A), two distinct unlabeled $[\mathrm{M}+2 \mathrm{H}]^{2+}$ ion conformers are observed as well as two distinct species for the singly-labeled ions. The unmodified peptide and singly-modified peptide ion conformers are observed at shorter and longer drift times, respectively. Doubly-modified peptide ion conformers are not observed in the $5 \times$ trials but were observed as significant species at higher label concentrations. Additionally, at the $5 \times$ label concentration, total monomeric peptide ions accounts for approximately $50 \%$ of the total peptide signal, with $\sim 36 \%$ of the total peptide signal attributed to unlabeled monomer. The rest of the peptide signal is comprised of multimeric structures (see Figure

3. Huntingtin N-terminal monomeric and multimeric structures destabilized by covalent modification of heteroatomic residues 
3.5D - F for range of dimer $\mathrm{m} / \mathrm{z}$ values as well as the Supporting Information for modified dimer 2D IMS-MS distributions). Total dimer, trimer, and tetramer species comprise $\sim 38 \%, \sim 9 \%$, and $\sim 3 \%$ of the total peptide signal, respectively. Each multimer exists in several states of covalent modification. It is noted that this technique is unable to determine the origin of multimers; that is, it cannot distinguish between species that are modified before or after association. For this reason, the effect of covalent modification on formation of multimeric species was calculated as a percent of total peptide species.
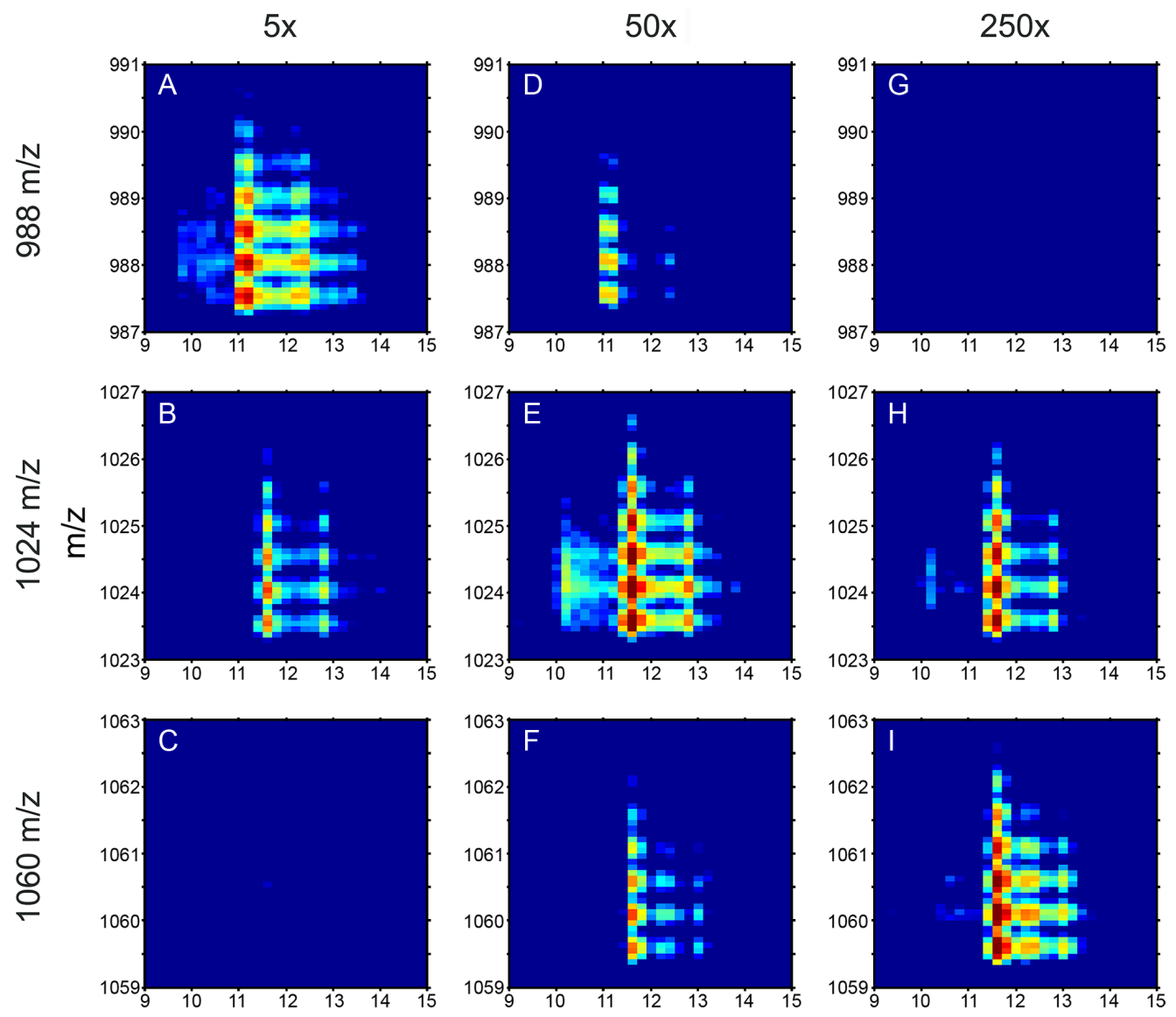

Drift Time (ms)

Figure 3.6. 2D IMS-MS distribution showing an expanded regions of unlabeled, singly-, and doubly-modified Nt17 at the 5x, 50x, and 250x DEPC concentrations. Note the appearance of the intermediate doubly-modified species $(1060 \mathrm{~m} / \mathrm{z}, 50 \mathrm{x}$ and 250x).

3. Huntingtin $\mathrm{N}$-terminal monomeric and multimeric structures destabilized by covalent modification of heteroatomic residues 
The first modification increases the collision cross section of $[\mathrm{M}+2 \mathrm{H}]^{2+}$ peptide conformers by $\sim 14 \AA^{2}$ (Figure 3.5A). Overall monomer signal increases at the $50 \times$ label concentration (Figure $3.5 \mathrm{E}$ ), to approximately $66 \%$ of the total peptide signal. At this concentration, a second modification is evident at $m / z$ 1060 (Figure 3.5B and 3.6F and I). The second modification has less effect on collision cross section; the largest increase in collision cross section was $\sim 7 \AA^{2}$ for the most extended conformer. Notably, a new conformer is observed at a $t_{D}$ of $12.2 \mathrm{~ms}\left(653 \AA^{2}\right)$ in the doubly-modified $[\mathrm{M}+2 \mathrm{H}]^{2+}$ ion $t_{D}$ distribution. It is noted that the size of this is closer to the more compact arrangement (Figure 3.3B, Structure 2). No additional collapsed monomeric structures are observed, and the small amount of organic solvent added to the solution to initiate the labeling was not sufficient to induce denaturation of the peptide (less than $1 \%$ by volume for each concentration); evidence for new structures was only observed once the peptide was doubly modified. Under these conditions, the majority of the monomer signal stems from the singly modified peptide; approximately $53 \%$ of the total peptide signal is attributed to this species alone. Multimer signal also decreases, falling to $\sim 27 \%, \sim 5 \%$, and $\sim 2 \%$ total dimer, trimer, and tetramer at the $50 \times$ level, respectively. 

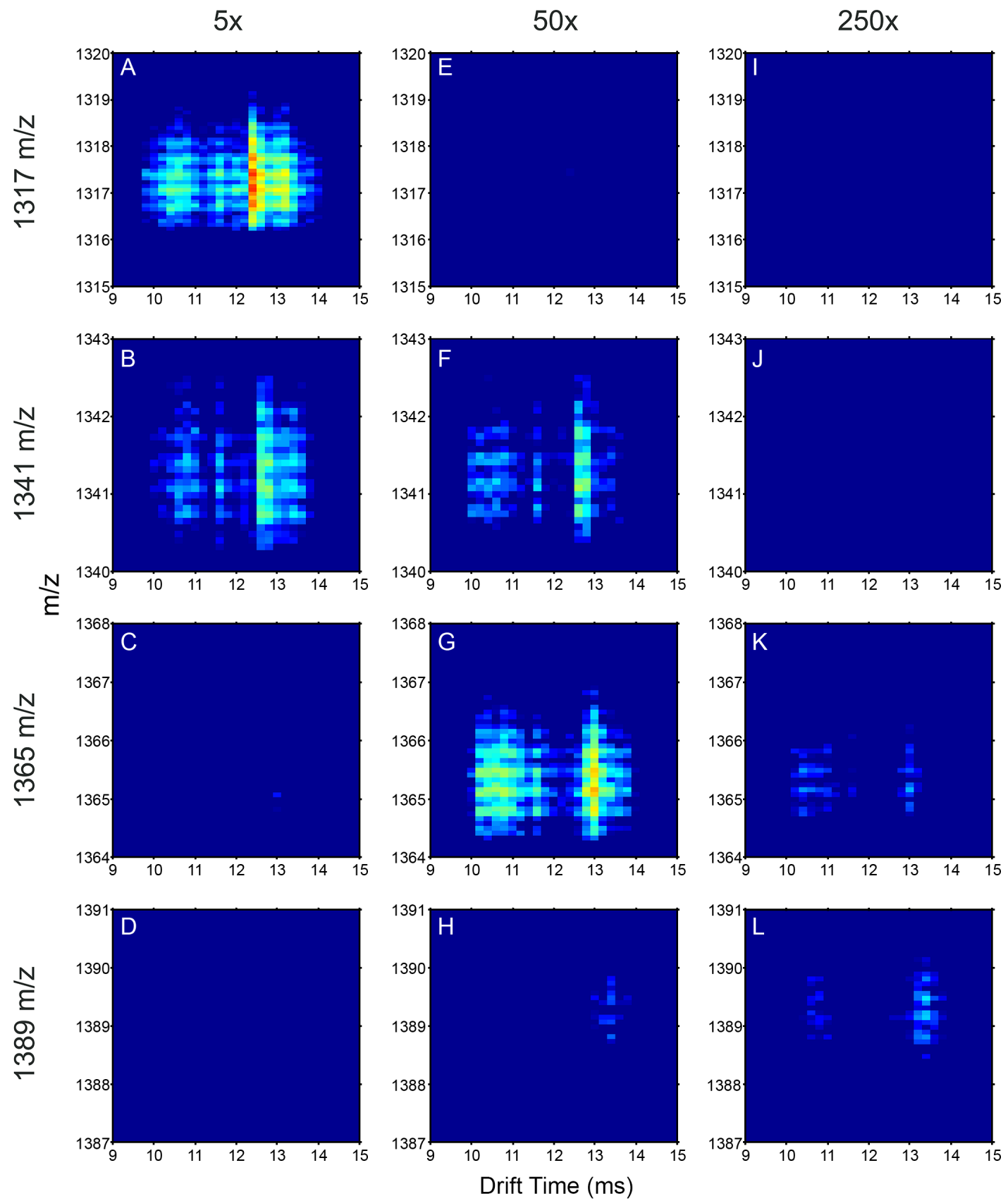

Figure 3.7. 2D IMS-MS distributions of modified dimer species. The overall abundance of mulitmer decreases as a function of label concentration.

At the $250 \times$ label concentration (Figure 3.5C and F, 3.6G, H, and I), almost no residual unlabeled monomer exists. The base peak of the collapsed

3. Huntingtin N-terminal monomeric and multimeric structures destabilized by covalent modification of heteroatomic residues 
mass spectrum is the doubly modified monomer. Total monomer increased in this experiment to $\sim 89 \%$ of the total peptide signal, while dimeric, trimeric, and tetrameric signal dropped to $\sim 8 \%, \sim 2 \%$, and $\sim 0.5 \%$, respectively. The appearance of the doubly-modified monomer does correlate with a sharp decline in multimer formation. This suggests that the covalent modification induces instability in the complex leading to a shift in equilibrium to the monomeric state. These results could have implications in posttranslational modification studies of huntingtin exon 1 , where modifications of hydrophilic residues have been shown to alter aggregation kinetics and morphologies, either by introducing a monomeric structural change or through inhibition of residue-specific interactions $14-16,72$

\subsubsection{Lysine-6 is Modified at Elevated DEPC Concentrations.}

Figure 3.8 shows a mobility-selected MS/MS spectrum of the new, doubly modified, intermediate monomer conformer at $12.2 \mathrm{~ms}$. The front and back gates in the drift tube have been set to allow only species with an arrival time distribution from 12.0 to $12.6 \mathrm{~ms}$. Mass selection and fragmentation was performed in the mass spectrometer. Figure 3.8 shows the sequence coverage obtained with MS/MS analysis. The Nt17 peptide contains several potential sites of modification, including the N-terminus, and residues $\mathrm{T} 3, \mathrm{~K} 6, \mathrm{~K} 9, \mathrm{~S} 14, \mathrm{~K} 15$, and S16. Singly modified peptides from MS/MS analysis are modified either at the Nterminus or at residue T3. Unfortunately, the $b_{1}$ and $b_{2}$ ions are not present in the 
observed ion sequence; therefore, the first modification could not be unambiguously identified. The $\mathrm{N}$-terminus would be the most likely site of

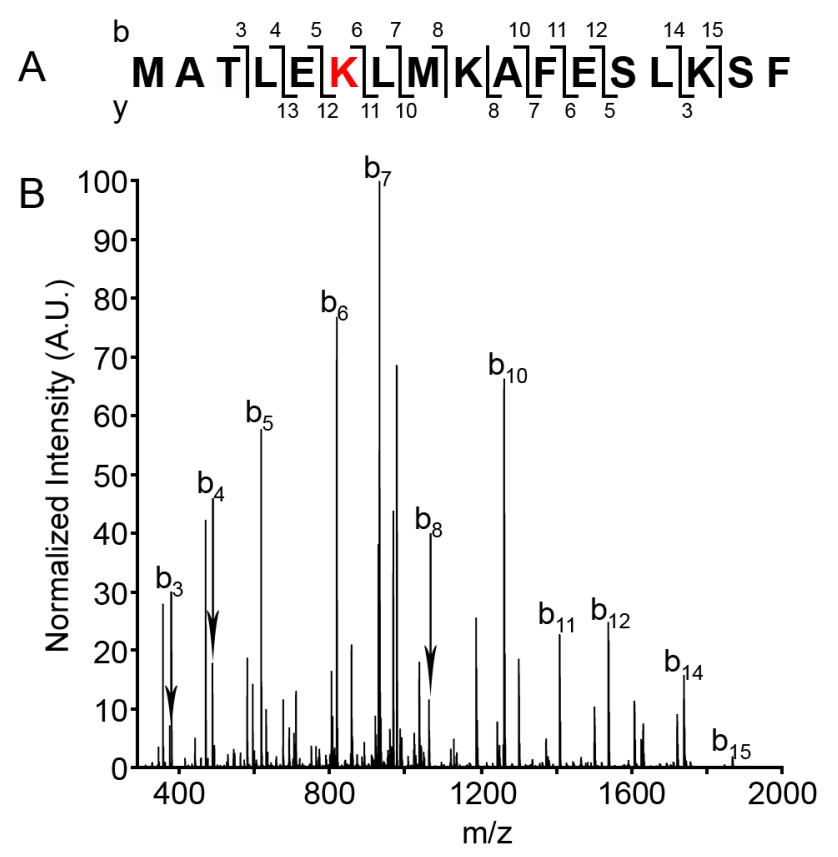

modification, because even in an aggregated state, it is solventexposed and heteroatom sites are very labile $5,13,52$. It would appear that the modification of residues $\mathrm{N}$-terminal to Leu4 does not introduce any structural rearrangement (Figure 3.5B, e, and $\mathrm{h}$ ); this modification has little

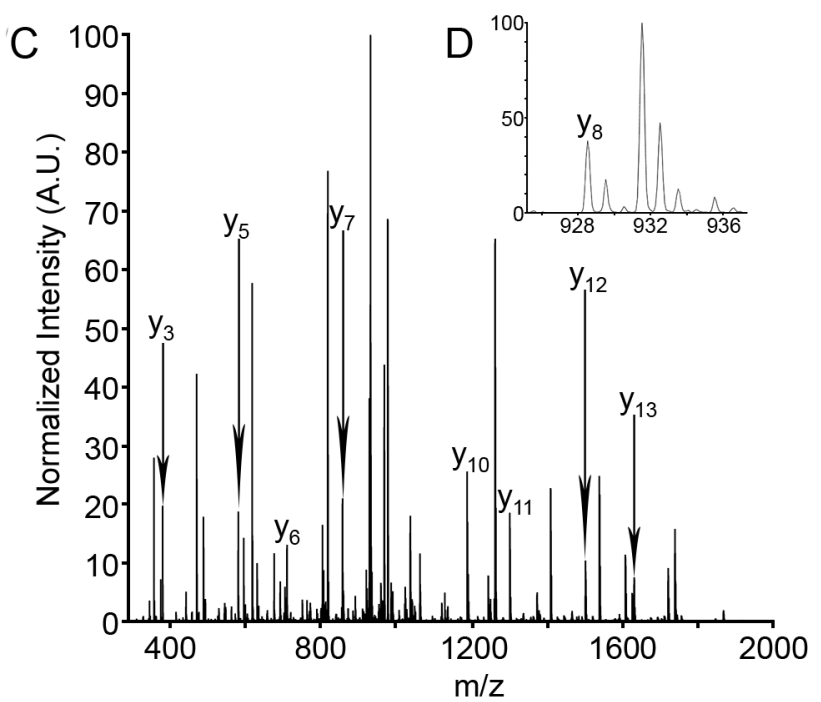
to no bearing on gas-phase conformation, other than a slight increase in collision crosssection owing to the larger carbethoxy moiety.

At elevated DEPC concentrations, $\mathrm{K} 6$ is modified. MS/MS studies confirm that all

Figure 3.8. Mobility selected MS/MS analysis of the intermediate, doubly modified conformer. a.) Sequence coverage by ion. b.) $b$ ion series. c.) y-ion series. d.) Enhanced region showing the $y_{8}$ ion for clarity.

three doubly modified

monomeric conformers are

modified at the same residues.

The $b_{7}$ ion is the most abundant ion in the MS/MS spectrum. Additionally, an ion

3. Huntingtin N-terminal monomeric and multimeric structures destabilized by 
corresponding to unmodified K6 was not observed. For these conformers, no other lysine residues were modified; carbethoxylation occurred exclusively on K6, and as such, resulted directly in the newly observed conformer at $12.2 \mathrm{~ms}$ (Figure 3.5F and I). $\mathrm{K} 6$ is contained within the conserved monomeric ion $\alpha$-helix in the N-terminal portion of Nt17 from MDS (Figure 3.3B and c, Structures 1 and 2). Additionally, MDS suggests that K6 could be directly involved in two stabilizing interactions in the Nt17 dimer; both $\mathrm{E} 12$ and $\mathrm{S} 16$ are potential binding partners for this residue. The most dramatic decrease in total mulitmer formation correlates with the emergence of the doubly-modified conformer at $t_{D} 12.2 \mathrm{~ms}$, which suggests this structural transition increases destabilization of multimeric species, either by introducing a new intermolecular interaction that destabilizes the $\alpha$-helix, or by preventing hydrophilic K6 interactions, such as those suggested by MDS.

\subsubsection{Interpretation of Structural Findings.}

Simulations of pathogenic and non-pathogenic Nt17-polyQ-polyP reveal that $\mathrm{Nt17}$ has the propensity to form helical bundles early in the aggregation process, with helical content increasing in the pathogenic $(55 \mathrm{Q})$ form ${ }^{69}$. The monomeric extended helix conformer has been implicated in studies containing the amyloidogenic polyGIn tract as the most prevalent structure, with helices ranging from residues $4-12$ to $2-17^{5,13,69,71}$. In contrast, solid state NMR studies on fully aggregated $\mathrm{Nt} 17 \mathrm{Q}_{35} \mathrm{P}_{10} \mathrm{~K}_{2}$ showed that $\beta$-sheet character from the amyloid core penetrated to S16; however, helical nature was conserved over

3. Huntingtin $\mathrm{N}$-terminal monomeric and multimeric structures destabilized by 
the region containing $\mathrm{K} 6{ }^{17}$. This is not out of the realm of possibility, as in light of the results obtained here, the random nature of the C-terminal portion of $\mathrm{Nt} 17$ suggested by MDS could very easily transition to a $\beta$-sheet conformation. Previous simulations on the Nt17 region alone show a two-helix bundle is a dominant structure at room temperature; residues Met1 - Met8 are contained in an $\alpha$-helix with a loop from residues $\mathrm{K} 9-\mathrm{F} 11^{71}$. In the same study, a second, straight helix conformer is evidenced at room temperature. The current study provides evidence for two relevant monomeric Nt17 structures stemming from solution species, which are mostly consistent with the findings of Kelley et al ${ }^{71}$. A strong helical propensity is observed for the first eleven $\mathrm{N}$-terminal residues; however, where Kelley et al. demonstrate a helix-turn-helix structure, referred to in their work as a two-helix bundle, the current study shows that the most abundant ion conformer contains a helical $\mathrm{N}$-terminus with a disordered $\mathrm{C}$ terminus. The second structure presented in the current study could most closely resemble the extended helix structure observed by Kelley et al., though they did not report any exivdence of a 3-10 helix. The data presented in the study by Kelley et al., and the work presented here, contrasts previously published work that states no stable helix is evident in $\mathrm{N}$-terminal huntingtin monomers ${ }^{5,73}$. It should be noted that measurements in these referenced studies are based on circular dichroism spectropolarimetry, which only reports on average characteristics. It was assumed that $\alpha$-helix character was a result of transient oligomers present in solution upon reconstitution. In light of the data presented here, this conclusion may be cursory, as both the matching monomeric and

3. Huntingtin N-terminal monomeric and multimeric structures destabilized by covalent modification of heteroatomic residues 
multimeric MDS conformations contain a degree of helicity. Indeed, crystal structures of huntingtin model peptides with an extended polyGln tract show a large amount of helicity in the $\mathrm{Nt} 17$ region ${ }^{68}$.

Trimeric and tetrameric species have been observed that appear to have conformers similar in size to bundles of elongated helices ${ }^{45}$. These structural assignments are in good agreement with prior work that states Nt17 associates via $\alpha$-helical interactions and maintains its helical nature in mature amyloid fibrils $5,13,17,71$. Because the data in the current study seem to indicate that elongated, helical, structures are conserved in the gas phase, it is noted that the elongated trimer and tetramer conformers observed here are assumed to contain a degree of helicity. This can also be argued due to the nature of Nt17 to form extended helices in amyloid fibrils and the similarity of collision cross sections to calculated helical bundle peptides of similar length.

\subsubsection{Covalent Modifications Alter Structures.}

Multiple PTM's have been implicated in accelerating or inhibiting huntingtin exon 1 aggregation ${ }^{14-16,74,75}$. Phosphorylation of T3 leads to decreased toxicity but increased aggregation rates ${ }^{16}$. Carbethoxylation of heteroatoms, while not a physiological PTM, is a means to increase hydrophobicity and introduces steric hindrance in the helical region. Excess of label reagent can destabilize secondary structures ${ }^{54}$. Previous studies have

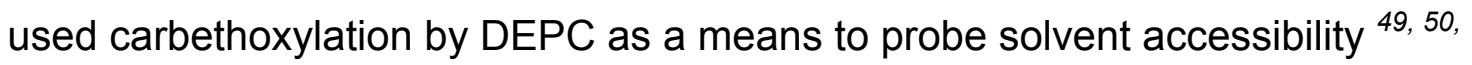

${ }^{54}$. Mendoza et al. use the reagent to track lysine, threonine, and histidine

3. Huntingtin $N$-terminal monomeric and multimeric structures destabilized by 
residues in isoforms of $\beta 2$ microglobulin ${ }^{49,50}$. In the present work, however, the modification of a single, hydrophilic residue led to a new monomer conformer and in concert, reduced overall multimerization. This occurs with a decrease in the elongated monomer conformer. The total contribution of the elongated conformer drops from $10 \%$ to approximately $7 \%$ as the new conformer appears. This may suggest that this conformer arises mainly from the elongated conformer, even though the majority of the doubly modified conformer still exists primarily in a compact conformation.

Previous work highlights the importance of $\mathrm{K} 6$ and its protection from an aggregated state in a huntingtin exon 1 model, indicating that $\mathrm{K} 6$ is involved in a stabilizing intra- or intermolecular interaction ${ }^{52}$. In previous studies, which utilized top-down deuterium exchange techniques, it was not possible to determine the exact origin of monomeric peptide. Results from the present study suggest that $\mathrm{K} 6$ is available in a monomeric state and may be involved in stabilizing multimer interactions, which would account for previous reports of decreased accessibility ${ }^{52}$. Modification of $\mathrm{K} 6$ in the peptide's multimeric state could reduce stabilizing interactions, and as a result, favor dissociation of the multimer species. Additionally, the modification could present a monomer structure that is not amenable for interaction mediated through $\mathrm{K} 6$ due to altered intramolecular interactions, once again preventing multimerization.

\footnotetext{
3. Huntingtin N-terminal monomeric and multimeric structures destabilized by
} 


\subsubsection{Implication of New Monomeric Structure.}

In some cases, post-translational modification can have accelerating effects on multimerization. For instance, phosphorylation of T3 in huntingtin exon 1 increased aggregation rate, as well as toxicity, in Drosophila models; Aiken et al. show that T3 phosphorylation occurs in vivo and may represent a therapeutic strategy for Huntington's disease remediation ${ }^{16}$. Two explanations for neuroprotection are proposed. Increased hydrophobicity of T3 due to modification either increases the rate of aggregation through the more toxic oligomeric state, thus reducing the amount of available toxic oligomer and leading to the neuroprotective fibrillar form. Conversely, modification of T3 destabilizes helical propensity and intermolecular association, thus preventing an oligomeric state to form entirely, leading to formation of fibrils that are not mediated through an oligomeric precursor. It should be noted that K6 lies on the boundary of the hydrophilic and hydrophobic faces, so the interpretation provided by Aiken et al. for T3 could also be extended to K6. That is, the data in the current study would suggest that the latter interpretation for neuroprotection proposed by Aiken et al.; an altered helix state does not allow for extended Nt17 multimers to form, promoting aggregation via a pathway that is not modulated by Nt17. Additionally, the T3 modification itself destabilizes quaternary arrangement leading to dissociation of multimer species. Since the model in the current study did not contain a polyGln tract, no comment on fibril formation can be made, nor can any conclusion be drawn regarding the effects of $\beta$-sheet formation in $\mathrm{Nt17}$ as a result of extended polyGln segments; however, it can be concluded that

3. Huntingtin $N$-terminal monomeric and multimeric structures destabilized by 
modification of Nt17 at a critical boundary lysine residue reduces multimerization, which could lead to altered aggregation pathways.

Multiple studies have reported on the propensity of $\mathrm{Nt} 17$ to form an $\alpha$-helix upon association with another Nt17 tract ${ }^{5,13}$ or a lipid bilayer ${ }^{7,76}$. Solid state NMR studies have shown that Nt17 is mostly helical in amyloid fibrils and retains its helicity even after advanced fibrillization, and that helicity increases with increased association ${ }^{13,17}$. Indeed, formation of an amphipathic $\alpha$-helix is a critical step in exon 1 nucleation ${ }^{5,44,67}$. Tam et al. have shown that an amphipathic helix from residues $4-12$ are necessary for advanced huntingtin fibrillization, which represents a more extended helix ${ }^{67}$. In the present study, a large portion of the monomer conformation appears to exist in an extended helical state in the gas phase and, upon labeling, is destabilized. It would appear that antiparallel association (Figure $3 \mathrm{C}$ ) of pre-elongated peptides is possible; however, it is unclear which (if either) conformation is the most disease-relevant. One interpretation is that destabilization of the pre-existing helix abrogates bundled multimer structure formation that resembles Nt17 nucleation in fulllength huntingtin exon 1. It could be that the more compact monomer conformer may not contribute as significantly to Nt17-mediated nucleation as the extended state due to the C-terminal coil region folding back into, and potentially interacting with, the helical region. 


\subsubsection{Implication of Lysine Modification.}

Depletion of the most elongated dimer conformers was observed upon modification of $\mathrm{K} 6$, which implies that $\mathrm{K} 6$ could be involved in more elongated helical associations of Nt17. Additionally, simulations of gas-phase dimer interactions show the involvement of K6 in stabilizing hydrogen bond interactions with oxygen-containing residues. Multiple solution simulations show that K6 is

contained in the helical portion of $\mathrm{Nt} 17$, whether in its monomeric form ${ }^{17,69}$ or bound to a lipid substrate ${ }^{6,7,76}$. While the current study did not probe aggregation kinetics, the study does suggest that modifying the $\varepsilon$-amino group on K6 inhibits inter-helical association. Modification of both the $\mathrm{N}$-terminal residue and K6 could work synergistically, by introducing steric hindrances that prohibit association and by altering secondary structure, to reduce multimer formation. Currently, it is not clear as to the mechanism of inhibition; however, K6 appears to be critical in Nt17 association.

Huntingtin Nt17 peptide monomer and multimer structures have been analyzed by IMS-MS and MDS. Direct observation of monomer structure reveals two populated solution states that correlate well with prior simulation and experiment, both of which could contain partial $\alpha$-helices. Several multimeric conformations are also observed, particularly for the dimer structure, each containing a degree of helicity. The most elongated dimer structure may consist of an arrangement of antiparallel $\alpha$ helices, and could be the most diseaserelevant conformation. This arrangement most closely resembles association of Nt17 in full-length huntingtin exon 1. MDS revealed intermolecular association in 
the elongated dimer ions is stabilized through K6-E12 and K6-S16 hydrogen bond interactions. Carbethoxylation of an $\mathrm{N}$-terminal residue and $\mathrm{K} 6$ may destabilize the helical structure as a new compact conformer is observed in the doubly-modified spectrum. Additionally, this modification drastically reduced multimer formation, which may suggest the critical role of $\mathrm{K} 6$ in the earliest stages of Nt17-mediated huntingtin exon 1 aggregation. 


\subsection{References}

(1) The Huntington's Disease Collaborative Research Group (1993) A NOVEL GENE CONTAINING A TRINUCLEOTIDE REPEAT THAT IS EXPANDED AND UNSTABLE ON HUNTINGTONS-DISEASE CHROMOSOMES, Cell 72, 971-983.

(2) Penney, J. B., Vonsattel, J. P., MacDonald, M. E., Gusella, J. F., and Myers, R. H. (1997) CAG repeat number governs the development rate of pathology in Huntington's disease, Annals of Neurology 41, 689-692.

(3) Mangiarini, L., Sathasivam, K., Seller, M., Cozens, B., Harper, A., Hetherington, C., Lawton, M., Trottier, Y., Lehrach, H., Davies, S. W., and Bates, G. P. (1996) Exon 1 of the HD gene with an expanded CAG repeat is sufficient to cause a progressive neurological phenotype in transgenic mice, Cell 87, 493-506.

(4) Jayaraman, M., Mishra, R., Kodali, R., Thakur, A. K., Koharudin, L. M. I., Gronenborn, A. M., and Wetzel, R. (2012) Kinetically Competing Huntingtin Aggregation Pathways Control Amyloid Polymorphism and Properties, Biochemistry 51, 2706-2716.

(5) Jayaraman, M., Kodali, R., Sahoo, B., Thakur, A. K., Mayasundari, A., Mishra, R., Peterson, C. B., and Wetzel, R. (2012) Slow Amyloid Nucleation via alpha-Helix-Rich Oligomeric Intermediates in Short Polyglutamine-Containing Huntingtin Fragments, Journal of Molecular Biology 415, 881-899.

(6) Côté, S., Wei, G., and Mousseau, N. (2014) Atomistic mechanisms of huntingtin $\mathrm{N}$-terminal fragment insertion on a phospholipid bilayer revealed by molecular dynamics simulations, Proteins: Structure, Function, and Bioinformatics, n/a-n/a.

(7) Michalek, M., Salnikov, E. S., Werten, S., and Bechinger, B. (2013) Membrane Interactions of the Amphipathic Amino Terminus of Huntingtin, Biochemistry 52, 847-858.

(8) Burke, K. A., Kauffman, K. J., Umbaugh, C. S., Frey, S. L., and Legleiter, J. (2013) The Interaction of Polyglutamine Peptides With Lipid Membranes is Regulated by Flanking Sequences Associated with Huntingtin, Journal of Biological Chemistry.

(9) Zheng, Z. Q., Li, A. M., Holmes, B. B., Marasa, J. C., and Diamond, M. I. (2013) An N-terminal Nuclear Export Signal Regulates Trafficking and Aggregation of Huntingtin (Htt) Protein Exon 1, Journal of Biological Chemistry 288, 6063-6071.

(10) Michalek, M., Salnikov, E. S., and Bechinger, B. (2013) Structure and Topology of the Huntingtin 117 Membrane Anchor by†a†Combined Solution and Solid-State NMR Approach, Biophysical journal 105, 699710.

(11) Giulia Rossetti, P. C., Alessandro Laio, Paolo Carloni. (2011) Conformations of the Huntingtin $\mathrm{N}$-term in aqueous solution from atomistic simulations, FEBS Letters, 3086 - 3089.

3. Huntingtin $\mathrm{N}$-terminal monomeric and multimeric structures destabilized by 
(12) Williamson, T. E., Vitalis, A., Crick, S. L., and Pappu, R. V. (2010) Modulation of Polyglutamine Conformations and Dimer Formation by the $\mathrm{N}-$ Terminus of Huntingtin, Journal of Molecular Biology 396, 1295-1309. Sivanandam, V. N., Jayaraman, M., Hoop, C. L., Kodali, R., Wetzel, R., and van der Wel, P. C. A. (2011) The Aggregation-Enhancing Huntingtin $\mathrm{N}$-Terminus Is Helical in Amyloid Fibrils, Journal of the American Chemical Society 133, 4558-4566.

(14) Mishra, R., Hoop, C. L., Kodali, R., Sahoo, B., van der Wel, P. C. A., and Wetzel, R. (2012) Serine Phosphorylation Suppresses Huntingtin Amyloid Accumulation by Altering Protein Aggregation Properties, Journal of Molecular Biology 424, 1-14.

(15) Gu, X. F., Greiner, E. R., Mishra, R., Kodali, R., Osmand, A., Finkbeiner, S., Steffan, J. S., Thompson, L. M., Wetzel, R., and Yang, X. W. (2009) Serines 13 and 16 Are Critical Determinants of Full-Length Human Mutant Huntingtin Induced Disease Pathogenesis in HD Mice, Neuron 64, 828840.

(16) Aiken, C. T., Steffan, J. S., Guerrero, C. M., Khashwji, H., Lukacsovich, T., Simmons, D., Purcell, J. M., Menhaji, K., Zhu, Y. Z., Green, K., LaFerla, F., Huang, L., Thompson, L. M., and Marsh, J. L. (2009) Phosphorylation of Threonine 3 IMPLICATIONS FOR HUNTINGTIN AGGREGATION AND NEUROTOXICITY, Journal of Biological Chemistry 284, 29427-29436.

(17) Hoop, C. L., Lin, H.-K., Kar, K., Hou, Z., Poirier, M. A., Wetzel, R., and van der Wel, P. C. A. (2014) Polyglutamine amyloid core boundaries and flanking domain dynamics in huntingtin fragment fibrils determined by solid-state NMR, Biochemistry.

(18) Kar, K., Hoop, C. L., Drombosky, K. W., Baker, M. A., Kodali, R., Arduini, I., van der Wel, P. C. A., Horne, W. S., and Wetzelt, R. (2013) betaHairpin-Mediated Nucleation of Polyglutamine Amyloid Formation, Journal of Molecular Biology 425, 1183-1197.

(19) Wyttenbach, T., von Helden, G., and Bowers, M. T. (1996) Gas-Phase Conformation of Biological Molecules: Bradykinin, Journal of the American Chemical Society 118, 8355-8364.

(20) Counterman, A. E., and Clemmer, D. E. (2002) Cis-Trans Signatures of Proline-Containing Tryptic Peptides in the Gas Phase, Analytical Chemistry 74, 1946-1951.

(21) Taraszka, J. A., Counterman, A. E., and Clemmer, D. E. (2001) Large anhydrous polyalanine ions: substitution of $\mathrm{Na}+$ for $\mathrm{H}+$ destabilizes folded states, International Journal of Mass Spectrometry 204, 87-100.

(22) Mao, Y., Woenckhaus, J., Kolafa, J., Ratner, M. A., and Jarrold, M. F. (1999) Thermal Unfolding of Unsolvated Cytochrome c: Experiment and Molecular Dynamics Simulations, Journal of the American Chemical Society 121, 2712-2721.

(23) Hall, Z., Politis, A., and Robinson, C. V. (2012) Structural modeling of heteromeric protein complexes from disassembly pathways and ion mobility-mass spectrometry, Structure 20, 1596-1609.

3. Huntingtin N-terminal monomeric and multimeric structures destabilized by covalent modification of heteroatomic residues 
(24) Politis, A., Park, A. Y., Hyung, S.-J., Barsky, D., Ruotolo, B. T., and Robinson, C. V. (2010) Integrating Ion Mobility Mass Spectrometry with Molecular Modelling to Determine the Architecture of Multiprotein Complexes, PLoS ONE 5, e12080.

(25) Ruotolo, B. T., Hyung, S.-J., Robinson, P. M., Giles, K., Bateman, R. H., and Robinson, C. V. (2007) Ion Mobility-Mass Spectrometry Reveals Long-Lived, Unfolded Intermediates in the Dissociation of Protein Complexes, Angewandte Chemie International Edition 46, 8001-8004.

(26) Gidden, J., Kemper, P. R., Shammel, E., Fee, D. P., Anderson, S., and Bowers, M. T. (2003) Application of ion mobility to the gas-phase conformational analysis of polyhedral oligomeric silsesquioxanes (POSS), International Journal of Mass Spectrometry 222, 63-73.

(27) Wyttenbach, T., and Bowers, M. T. (2011) Structural Stability from Solution to the Gas Phase: Native Solution Structure of Ubiquitin Survives Analysis in a Solvent-Free Ion Mobility-Mass Spectrometry Environment, Journal of Physical Chemistry B 115, 12266-12275.

(28) Pierson, N. A., Chen, L., Valentine, S. J., Russell, D. H., and Clemmer, D. E. (2011) Number of Solution States of Bradykinin from Ion Mobility and Mass Spectrometry Measurements, Journal of the American Chemical Society 133, 13810-13813.

(29) Bernstein, S. L., Dupuis, N. F., Lazo, N. D., Wyttenbach, T., Condron, M. M., Bitan, G., Teplow, D. B., Shea, J. E., Ruotolo, B. T., Robinson, C. V., and Bowers, M. T. (2009) Amyloid-beta protein oligomerization and the importance of tetramers and dodecamers in the aetiology of Alzheimer's disease, Nature Chemistry 1, 326-331.

(30) Shi, H. L., Pierson, N. A., Valentine, S. J., and Clemmer, D. E. (2012) Conformation Types of Ubiquitin $\mathrm{M}+8 \mathrm{H}(8+)$ lons from Water:Methanol Solutions: Evidence for the $\mathrm{N}$ and $\mathrm{A}$ States in Aqueous Solution, Journal of Physical Chemistry B 116, 3344-3352.

(31) Shi, L., Holliday, A. E., Shi, H., Zhu, F., Ewing, M. A., Russell, D. H., and Clemmer, D. E. (2014) Characterizing Intermediates Along the Transition from Polyproline I to Polyproline II Using Ion Mobility Spectrometry-Mass Spectrometry, Journal of the American Chemical Society.

(32) Hudgins, R. R., Woenckhaus, J., and Jarrold, M. F. (1997) High resolution ion mobility measurements for gas phase proteins: correlation between solution phase and gas phase conformations, International Journal of Mass Spectrometry and lon Processes 165-166, 497-507.

(33) Clemmer, D. E., Hudgins, R. R., and Jarrold, M. F. (1995) Naked Protein Conformations: Cytochrome $\mathrm{c}$ in the Gas Phase, Journal of the American Chemical Society 117, 10141-10142.

(34) Bleiholder, C., Do, T. D., Wu, C., Economou, N. J., Bernstein, S. S., Buratto, S. K., Shea, J. E., and Bowers, M. T. (2013) Ion Mobility Spectrometry Reveals the Mechanism of Amyloid Formation of A beta(2535) and Its Modulation by Inhibitors at the Molecular Level:

3. Huntingtin N-terminal monomeric and multimeric structures destabilized by 
Epigallocatechin Gallate and Scyllo-inositol, Journal of the American Chemical Society 135, 16926-16937.

(35) Li, H., Bendiak, B., Siems, W. F., Gang, D. R., and Hill, H. H. (2013) Carbohydrate Structure Characterization by Tandem lon Mobility Mass Spectrometry (IMMS)2, Analytical Chemistry 85, 2760-2769.

(36) Kloniecki, M., Jablonowska, A., Poznanski, J., Langridge, J., Hughes, C., Campuzano, I., Giles, K., and Dadlez, M. (2011) Ion Mobility Separation Coupled with MS Detects Two Structural States of Alzheimer's Disease A beta 1-40 Peptide Oligomers, Journal of Molecular Biology 407, 110-124.

(37) Bornschein, R. E., Hyung, S. J., and Ruotolo, B. T. (2011) lon MobilityMass Spectrometry Reveals Conformational Changes in Charge Reduced Multiprotein Complexes, Journal of the American Society for Mass Spectrometry 22, 1690-1698.

(38) Trimpin, S., Tan, B., Bohrer, B. C., O'Dell, D. K., Merenbloom, S. I., Pazos, M. X., Clemmer, D. E., and Walker, J. M. (2009) Profiling of phospholipids and related lipid structures using multidimensional ion mobility spectrometry-mass spectrometry, International Journal of Mass Spectrometry 287, 58-69.

(39) Shvartsburg, A. A., Li, F. M., Tang, K. Q., and Smith, R. D. (2006) Characterizing the structures and folding of free proteins using 2-D gasphase separations: Observation of multiple unfolded conformers, Analytical Chemistry 78, 3304-3315.

(40) Ridenour, W. B., Kliman, M., McLean, J. A., and Caprioli, R. M. (2010) Structural Characterization of Phospholipids and Peptides Directly from Tissue Sections by MALDI Traveling-Wave Ion Mobility-Mass Spectrometry, Analytical Chemistry 82, 1881-1889.

(41) Campuzano, I., Bush, M. F., Robinson, C. V., Beaumont, C., Richardson, K., Kim, H., and Kim, H. I. (2011) Structural Characterization of Drug-like Compounds by lon Mobility Mass Spectrometry: Comparison of Theoretical and Experimentally Derived Nitrogen Collision Cross Sections, Analytical Chemistry 84, 1026-1033.

(42) Hoaglund, C. S., Valentine, S. J., Sporleder, C. R., Reilly, J. P., and Clemmer, D. E. (1998) Three-Dimensional Ion Mobility/TOFMS Analysis of Electrosprayed Biomolecules, Analytical Chemistry 70, 2236-2242.

(43) Wu, C., Siems, W. F., Asbury, G. R., and Hill, H. H. (1998) Electrospray Ionization High-Resolution Ion Mobility Spectrometry-Mass Spectrometry, Analytical Chemistry 70, 4929-4938.

(44) Mishra, R., Jayaraman, M., Roland, B. P., Landrum, E., Fullam, T., Kodali, R., Thakur, A. K., Arduini, I., and Wetzel, R. (2012) Inhibiting the Nucleation of Amyloid Structure in a Huntingtin Fragment by Targeting alpha-Helix-Rich Oligomeric Intermediates, Journal of Molecular Biology 415, 900-917.

(45) Counterman, A. E., Valentine, S. J., Srebalus, C. A., Henderson, S. C., Hoaglund, C. S., and Clemmer, D. E. (1998) High-order structure and dissociation of gaseous peptide aggregates that are hidden in mass

3. Huntingtin N-terminal monomeric and multimeric structures destabilized by covalent modification of heteroatomic residues 
spectra, Journal of the American Society for Mass Spectrometry 9, 743759.

(46) Gessel, M. M., Wu, C., Li, H. Y., Bitan, G., Shea, J. E., and Bowers, M. T. (2012) A beta(39-42) Modulates A beta Oligomerization but Not Fibril Formation, Biochemistry 51, 108-117.

(47) Bleiholder, C., Dupuis, N. F., Wyttenbach, T., and Bowers, M. T. (2011) Ion mobility-mass spectrometry reveals a conformational conversion from random assembly to beta-sheet in amyloid fibril formation, Nature Chemistry 3, 172-177.

(48) Bernstein, S. L., Wyttenbach, T., Baumketner, A., Shea, J. E., Bitan, G., Teplow, D. B., and Bowers, M. T. (2005) Amyloid beta-protein: Monomer structure and early aggregation states of $A$ beta 42 and its Pro(19) alloform, Journal of the American Chemical Society 127, 2075-2084.

(49) Mendoza, V. L., Antwi, K., Baron-Rodriguez, M. A., Blanco, C., and Vachet, R. W. (2010) Structure of the Preamyloid Dimer of beta-2Microglobulin from Covalent Labeling and Mass Spectrometry, Biochemistry 49, 1522-1532.

(50) Mendoza, V. L., Baron-Rodriguez, M. A., Blanco, C., and Vachet, R. W. (2011) Structural Insights into the Pre-Amyloid Tetramer of beta-2Microglobulin from Covalent Labeling and Mass Spectrometry, Biochemistry 50, 6711-6722.

(51) Stocks, B. B., Rezvanpour, A., Shaw, G. S., and Konermann, L. (2011) Temporal Development of Protein Structure during S100A11 Folding and Dimerization Probed by Oxidative Labeling and Mass Spectrometry, Journal of Molecular Biology 409, 669-679.

(52) Arndt, J. R., Brown, R. J., Burke, K. A., Legleiter, J., and Valentine, S. J. (2015) Lysine residues in the $\mathrm{N}$-terminal huntingtin amphipathic $\alpha$-helix play a key role in peptide aggregation, Journal of Mass Spectrometry 50 , 117-126.

(53) Mendoza, V. L., and Vachet, R. W. (2009) Probing protein structure by amino acid-specific covalent labeling and mass spectrometry, Mass Spectrometry Reviews 28, 785-815.

(54) Mendoza, V. L., and Vachet, R. W. (2008) Protein Surface Mapping Using Diethylpyrocarbonate with Mass Spectrometric Detection, Analytical Chemistry 80, 2895-2904.

(55) Donohoe, G. C., Maleki, H., Arndt, J. R., Khakinejad, M., Yi, J., McBride, C., Nurkiewicz, T. R., and Valentine, S. J. (2014) A New Ion MobilityLinear Ion Trap Instrument for Complex Mixture Analysis, Analytical Chemistry 86, 8121-8128.

(56) Khakinejad, M., Kondalaji, S. G., Maleki, H., Arndt, J. R., Donohoe, G. C., and Valentine, S. J. (2014) Combining lon Mobility Spectrometry with Hydrogen-Deuterium Exchange and Top-Down MS for Peptide Ion Structure Analysis, Journal of The American Society for Mass Spectrometry 25, 2103-2115.

3. Huntingtin N-terminal monomeric and multimeric structures destabilized by 
(57) Kim, T., Tolmachev, A. V., Harkewicz, R., Prior, D. C., Anderson, G., Udseth, H. R., Smith, R. D., Bailey, T. H., Rakov, S., and Futrell, J. H. (2000) Design and Implementation of a New Electrodynamic Ion Funnel, Analytical Chemistry 72, 2247-2255.

(58) Wang, F. B., J. P.; Cieplak, P.; Dupradeau, F. Y. (2013) R.E.D. Python: Object oriented programming for Amber force fields, Université de Picardie - Jules Verne, Sanford|Burnham Medical Research Institute.

(59) Bayly, C. I., Cieplak, P., Cornell, W. D., and Kollman, P. A. (1993) A WELL-BEHAVED ELECTROSTATIC POTENTIAL BASED METHOD USING CHARGE RESTRAINTS FOR DERIVING ATOMIC CHARGES THE RESP MODEL, Journal of Physical Chemistry 97, 10269-10280.

(60) Schmidt, M. W., Baldridge, K. K., Boatz, J. A., Elbert, S. T., Gordon, M. S., Jensen, J. H., Koseki, S., Matsunaga, N., Nguyen, K. A., Su, S. J., Windus, T. L., Dupuis, M., and Montgomery, J. A. (1993) GENERAL ATOMIC AND MOLECULAR ELECTRONIC-STRUCTURE SYSTEM, Journal of Computational Chemistry 14, 1347-1363.

(61) Gordon, M. S., and Schmidt, M. W. (2005) Advances in electronic structure theory: GAMESS a decade later, In Theory and Applications of Computational Chemistry: the first forty years (Dykstra, C. E., Frenking, G., Kim, K. S., and Scuseria, G. E., Eds.), pp 1167-1189, Elsevier.

(62) Wyttenbach, T., vonHelden, G., and Bowers, M. T. (1996) Gas-phase conformation of biological molecules: Bradykinin, Journal of the American Chemical Society 118, 8355-8364.

(63) Case, D. A. D., T. A.; Cheatham, III, T. E.; Simmerling, C. L.; Wang, L.; Duke, R. E.; Luo, R.; Walker, R. C.; Zhang, W.; Merz, K. M.; Roberts, B.; Hayik, S.; Roitberg, A.; Seabra, G.; Swails, J.; Götz, A. W.; Kolossváry, I.; Wong, K. F.; Paesani, F.; Vanicek, J.; Wolf, R. M.; Liu, J.; Wu, X.; Brozell, S. R.; Steinbrecher, T.; Gohlke, H.; Cai, Q.; Ye, X.; Wang, J.; Hsieh, M. J.; Cui, G.; Roe, D. R.; Mathews, D. H.; Seetin, M. G.; Salomon-Ferrer, R.; Sagui, C.; Babin, V.; Luchko, T.; Gusarov, S.; Kovalenko, A.; Kollman, P. A. . (2012) Amber 12, University of California, San Francisco.

(64) Jarrold Research Group, I. U. MOBCAL - A Program to Calculate Mobilities.

(65) Mesleh, M. F., Hunter, J. M., Shvartsburg, A. A., Schatz, G. C., and Jarrold, M. F. (1996) Structural Information from Ion Mobility Measurements: Effects of the Long-Range Potential, The Journal of Physical Chemistry 100, 16082-16086.

(66) Bugg, C. W., Isas, J. M., Fischer, T., Patterson, P. H., and Langen, R. (2012) Structural Features and Domain Organization of Huntingtin Fibrils, Journal of Biological Chemistry 287, 31739-31746.

(67) Tam, S., Spiess, C., Auyeung, W., Joachimiak, L., Chen, B., Poirier, M. A., and Frydman, J. (2009) The chaperonin TRiC blocks a huntingtin sequence element that promotes the conformational switch to aggregation, Nature Structural \& Molecular Biology 16, 1279-U1298.

3. Huntingtin N-terminal monomeric and multimeric structures destabilized by 
(68) Kim, M. W., Chelliah, Y., Kim, S. W., Otwinowski, Z., and Bezprozvanny, I. (2009) Secondary Structure of Huntingtin Amino-Terminal Region, Structure 17, 1205-1212.

(69) Dlugosz, M., and Trylska, J. (2011) Secondary Structures of Native and Pathogenic Huntingtin N-Terminal Fragments, Journal of Physical Chemistry B 115, 11597-11608.

(70) Rossetti, G., Cossio, P., Laio, A., and Carloni, P. (2011) Conformations of the Huntingtin $\mathrm{N}$-term in aqueous solution from atomistic simulations, Febs Letters 585, 3086-3089.

(71) Kelley, N. W., Huang, X., Tam, S., Spiess, C., Frydman, J., and Pande, V. S. (2009) The Predicted Structure of the Headpiece of the Huntingtin Protein and Its Implications on Huntingtin Aggregation, Journal of Molecular Biology 388, 919-927.

(72) O'Rourke, J. G., Gareau, J. R., Ochaba, J., Song, W., Rasko, T., Reverter, D., Lee, J., Monteys, A. M., Pallos, J., Mee, L., Vashishtha, M., Apostol, B. L., Nicholson, T. P., Illes, K., Zhu, Y. Z., Dasso, M., Bates, G. P., Difiglia, M., Davidson, B., Wanker, E. E., Marsh, J. L., Lima, C. D., Steffan, J. S., and Thompson, L. M. (2013) SUMO-2 and PIAS1 Modulate Insoluble Mutant Huntingtin Protein Accumulation, Cell Reports 4, 362-375.

(73) Thakur, A. K., Jayaraman, M., Mishra, R., Thakur, M., Chellgren, V. M., Byeon, I. J. L., Anjum, D. H., Kodali, R., Creamer, T. P., Conway, J. F., Gronenborn, A. M., and Wetzel, R. (2009) Polyglutamine disruption of the huntingtin exon $1 \mathrm{~N}$ terminus triggers a complex aggregation mechanism, Nature Structural \& Molecular Biology 16, 380-389.

(74) Atwal, R. S., Desmond, C. R., Caron, N., Maiuri, T., Xia, J. R., Sipione, S., and Truant, R. (2011) Kinase inhibitors modulate huntingtin cell localization and toxicity, Nature Chemical Biology 7, 453-460.

(75) Thompson, L. M., Aiken, C. T., Kaltenbach, L. S., Agrawal, N., Illes, K., Khoshnan, A., Martinez-Vincente, M., Arrasate, M., O'Rourke, J. G., Khashwji, H., Lukacsovich, T., Zhu, Y. Z., Lau, A. L., Massey, A., Hayden, M. R., Zeitlin, S. O., Finkbeiner, S., Green, K. N., LaFerla, F. M., Bates, G., Huang, L., Patterson, P. H., Lo, D. C., Cuervo, A. M., Marsh, J. L., and Steffan, J. S. (2009) IKK phosphorylates Huntingtin and targets it for degradation by the proteasome and lysosome, Journal of Cell Biology 187, 1083-1099.

(76) Nagarajan, A., Jawahery, S., and Matysiak, S. (2013) The Effects of Flanking Sequences in the Interaction of Polyglutamine Peptides with a Membrane Bilayer, The Journal of Physical Chemistry B.

3. Huntingtin N-terminal monomeric and multimeric structures destabilized by 


\section{Polyproline peptides interact with $\mathrm{Nt17}$ and suppress}

\section{huntingtin aggregation}

The peptide representing the $\mathrm{Nt} 17$ region of htt has been shown in previous chapters to form stable elongated homodimeric and homomultimeric complexes in the gas phase. Association appears to be predicated on the ability of Nt17 to form an extended amphipathic a-helix. The C-terminal proline-rich region of htt exon 1 could possibly serve as a regulator of Nt17 in native htt exon 1 ; this interaction is disrupted due to a gain of rigidity in extended disease-length polyQ regions. ${ }^{1}$ The exact mechanism of $\mathrm{Nt} 17$ regulation by polyP is not well understood. Additionally, since polyP may act as a stabilizer of Nt17 in native htt, a co-incubation of disease-length htt exon 1 with a polyP peptide could have an impact on aggregation kinetics, morphology, or both, through interaction with Nt17. The work described in this chapter explores Nt17-polyP interactions through IMS-MS coupled to gas-phase HDX and MDS. A morphological effect of co-incubation is demonstrated by AFM. The results of this study indicate that polyP may regulate $\mathrm{Nt17}$ by stabilizing a random coil region after residue M8 in $\mathrm{Nt17}$, thus, not allowing the transition to the reactive amphipathic $\alpha$-helix.

\subsection{Introduction: PolyP as a regulator of $\mathrm{Nt17}$}

Nt17 modulates oligomerization of htt exon 1 , in bulk solution and in the presence of a lipid bilayer. ${ }^{2-4}$ Several studies have sought to block Nt17 selfinteraction as a means of preventing amyloid formation, or have shown that chemical modification results in aggregation pathways that may not Nt17- 
mediated and bypass the oligomeric state. ${ }^{5-8}$ One hypothesis suggests that the PolyP PPII helix acts as an intrinsic regulator for Nt17, and at sufficient PolyQ lengths, this interaction becomes less favorable due to induced $\beta$-turn strand structure in polyQ. ${ }^{1} \mathrm{~A}$ gain of structure, and thus, rigidity, in the polyQ region increases the intermolecular distance between Nt17 and PolyP, thus freeing Nt17 for disease-related interaction, such as association with other Nt17 tracts or interaction with cellular surfaces. ${ }^{1}$ Additionally, the polyP region could act synergistically with Nt17 to decrease solubility of the polyQ tract and thus, drive aggregation. ${ }^{9,10}$ Similarly, recent studies show that an Nt17 peptide co-incubated with an htt exon 1 peptide mimic was sufficient to force aggregation via a pathway not mediated by Nt17. It stands to reason, then, that like an Nt17 peptide, a polyP peptide could interact with Nt17 and perhaps modify aggregation, or at least promote a pathway that is not mediated by Nt17. To date, no technique has directly probed residue-specific Nt17-polyP interactions, nor has a structural model for interaction been proposed. The information gained by studying this interaction could lead to valuable insight to an important active region for Nt17-Nt17 association, and by extension, provide a new structural target for disease amelioration. This study uses hybrid ion mobility-mass spectrometry (IMS-MS) coupled to gas-phase hydrogen-deuterium exchange (HDX) to probe the interactions between Nt17 and polyP in the context of regulating Nt17 reactivity toward mediating aggregation. 
Nt17 is a strong promoter of both oligomer formation and amyloid formation; ${ }^{3,4,10}$ however, the location and extent of helicity in Nt17 is not well defined. In x-ray crystal structures of htt exon $1, \mathrm{Nt17}$ is entirely helical with the $\alpha$ helix extending into the polyQ region. ${ }^{11}$ In other solid state NMR and simulation experiments, the helix is not present in the monomeric form, but rather forms a helix when in proximity with a binding partner, such as a second Nt17 sequence or a lipid bilayer. ${ }^{12-14}$ In other cases, Nt17 is tightly associated with the polyQ region in a monomeric form. ${ }^{10}$ In any case, it appears that Nt17 is mostly helical in amyloid fibrils; ${ }^{4,14,15}$ thus, it represents an intriguing structural target for the amelioration of HD progression. Several studies have modified Nt17 through S to D mutation, which mimics phosphorylation, at serines 13 and $16{ }^{7,16}$ These mutations slowed the progression of Nt17-mediated htt aggregation, but did not prevent aggregation all together. While the goal of these studies were to examine the role of post-translational modification on aggregation, they did provide a framework for targeting Nt17. Similarly, Nt17 attached to a pathogenic polyQ tract has been targeted with a simple Nt17 peptide ${ }^{5}$ or by elimination by enzymatic digestion. ${ }^{3}$ This study showed a net decrease in aggregation, most likely due to Nt17-Nt17 interactions where the non-pathogenic fragment is included in the nucleation of pathogenic peptides, increasing the distance between polyQ fragments and thus, increasing the energy barrier required for amyloid formation. A similar idea, then, is to target the $\mathrm{Nt} 17$ region using another peptide. Ideally, this peptide would function the same as the Nt17 peptide alone in inhibiting nucleated amyloid formation. 
Previous work has shown that, in its native form, Nt17 may be stabilized by the polyP region, for non-pathogenic $\mathrm{Q}$-lengths. ${ }^{1}$ At disease-length poly $\mathrm{Q}$ tracts, the poly $Q$ region gains rigidity through $\beta$-sheet formation and increases the intermolecular distance between Nt17 and polyP, thus allowing Nt17 to mediate fibril nucleation; however, the structural mechanism by which polyP stabilizes Nt17 is unknown. The interaction could be modulated through $\mathrm{S} 13$ and S16, as phosphomimicry and true phosphorylation both showed an increase in intermolecular distance. ${ }^{1}$ Using IMS-MS, the current study aims to gain insight into the Nt17-polyP interactions and determine if polyP is a suitable inhibitor of htt exon 1 aggregation, much like co-incubation with other peptides that target Nt17.

\subsection{Materials and Methods}

\subsubsection{Sample Preparation.}

Nt17 (MATLEKLMKAFESLKSF) and polyP $\left(\right.$ Ac- $\left.\mathrm{P}_{10}-\mathrm{NH}_{2}\right)$ peptides were prepared according to established protocols, ${ }^{17}$ as described in Chapters 2 and 3. All samples were reconstituted in $500 \mu \mathrm{L}$ of $0.200 \mathrm{M}$ ammonium acetate (Fisher Scientific) in $18 \mathrm{M} \Omega$ water, purified using a Milli-Q purification system (Millipore). For binary samples, the first peptide was reconstituted, incubated, and vortexed. The resulting solution was then removed from the tube and used to reconstitute the second peptide film.

\subsubsection{Ion Mobility - Mass Spectrometry.}

Ion Mobility - Mass Spectrometry (IMS-MS) was performed on a homebuilt hybrid instrument that consists of a linear drift tube coupled to a Thermo 
LTQ Velos Pro (Thermo Scientific, San Jose, CA). Design, operation, and figures of merit for the instrument have been described elsewhere, and in Chapter $1 .{ }^{18}$ All samples were introduced at $0.5 \mu \mathrm{L} / \mathrm{min}$. lons were pulsed into the drift tube every $20 \mathrm{~ms}$ using a $150 \mu$ s pulse width. The time-delayed gate at the rear of the drift tube just before the mass spectrometer, was periodically lowered for $150 \mu \mathrm{s}$ at a rate of $50 \mathrm{~Hz}$. The delay between the front and rear gates is scanned to produce the drift spectrum. lons whose drift times are equal to the delay time are allowed to enter the mass spectrometer. The pressure in the drift tube was maintained at approximately 2.6Torr. The ion trap MS was set to scan every 400 $\mu \mathrm{s}$, and average every four microscans. Each mass spectrum in the drift tube experiment was collected for thirty seconds.

\subsubsection{Complex Energetics.}

Collision-induced dissociation (CID) was used to determine relative complex strength for each Nt17-PolyP multimer conformation. For comparison, Nt17 homodimer conformations were also sampled. Conformers were driftselected using the IMS. Gate timing was set in order to sample the entire peak. Mass selection and fragmentation was then performed in the mass spectrometer. Helium was used as the collision gas. The parent ion signal for the complex was monitored as a function of normalized collision energy. 


\subsubsection{Gas-phase Hydrogen-Deuterium Exchange (GP-HDX).}

Gaseous $D_{2} \mathrm{O}$ was introduced to the drift tube buffer gas to determine accessible sites to decipher protection as a result of non-covalent interaction, and to probe the most accessible sites in the complex, as described in Chapter 1. Liquid $\mathrm{D}_{2} \mathrm{O}$ was measured into a stainless steel bulb, sealed with a Swagelok fitting, and flash frozen using liquid nitrogen. The headspace above the $D_{2} \mathrm{O}$ was pumped away, leaving only $\mathrm{D}_{2} \mathrm{O}$ in the bulb. $\mathrm{D}_{2} \mathrm{O}$ was allowed into the drift tube using a bleed valve. The partial pressure of $\mathrm{D}_{2} \mathrm{O}$ for all experiments was 0.01 Torr. The helium pressure was adjusted to compensate for the addition of $D_{2} \mathrm{O}$, in order to maintain a total pressure of 2.61 Torr. This is a sufficient amount of the reagent to induce labeling of rapidly exchanging sites; in this manner, only the most accessible sites are labeled.

\subsubsection{Molecular Dynamics Simulations.}

Molecular dynamics simulations (MD) and simulated annealing were performed as described in Chapter 3, using the same force field and molecular constraints. PolyP was constrained to an all trans conformation in AMBER by limiting the $\omega$ angle to between $150^{\circ}$ and $210^{\circ}$. An all-trans proline conformation was chosen because polyP tracts favor all-trans PPII helices in aqueous solvents, ${ }^{19}$ and the polyP tract in htt exon 1 forms a PPII-helix. ${ }^{20,21}$ Simulated annealing was performed as described previously in Chapter 3 for two different starting structures. The lowest energy conformations within $\pm 1 \%$ of the measured CCS were examined using a hydrogen accessibility scoring (HAS) model. ${ }^{22}$ 


\subsubsection{Hydrogen accessibility scoring and kinetics model.}

Hydrogen accessibility scoring (HAS) was performed for each structure according to methods developed in the Valentine lab. ${ }^{22}$ This algorithm was used to validate the candidate structures based on the measured deuterium uptake. To calculate the HAS, each carbonyl oxygen within $7 \AA$ of a charge site, scaled as the inverse distance to the charge site $\left(d_{\mathrm{O}-\mathrm{N}}\right)$ was scored $\left(S_{\mathrm{O}}\right)$. This is the HDXactive carbonyl that initiates deuterium exchange. In the event multiple charge sites were within the proximity of a carbonyl, the oxygen was scored as the sum of inverse distances. Next, each heteroatomic hydrogen within $7 \AA$ of the active carbonyl was scored as:

$$
S_{H}=\sum \frac{S_{O}}{d_{H-O}}
$$

where $S_{H}$ is the hydrogen score and $d_{H-O}$ is the interatomic distance between the hydrogen and the HDX active carbonyl. The residue score $\left(S_{R}\right)$ was determined using Equation 4.2:

$$
S_{R}=\sum S_{H}
$$

Solvent accessibility was also assessed in silico by allowing a water molecue to sample the entire peptide's surface to obtain an excluded volume. The solvent accessibility and the HAS were weighted equally in calculations of exchange 
kinetics for fast exchanging hydrogens. Exchange kinetics were calculated using Equation 4.3:

$$
\begin{array}{ll}
1-\frac{A}{A_{0}} & \\
& \\
& \text { where } \\
& \\
\frac{A}{A_{0}}=e^{-k t_{i}} & 4.3 .4 .
\end{array}
$$

The rate constant $k$ is estimated using values recorded for positively charged peptide ions. ${ }^{22,}{ }^{23}$ In Equation 4.3, $A / A_{0}$ represents the fraction of sites that do not exchange at a given time $t_{i}$. Fractions altered were compared to a ratio of a random number to total possible random numbers generated. If the ratio was less than the fraction altered, then an exchange event was recorded for the exchange site. The process was repeated as the ion stepped through the time increment $\left(t_{i}\right)$ up to the final drift time.

\subsubsection{Purification of htt exon 1 construct.}

Disease-length (51Q) htt exon 1 was purified from Escherichia coli according to previously published protocols. ${ }^{17,}{ }^{24}$ Briefly, pelleted E. coli colonies containing glutathione S-transferase (GST) fused htt exon 1 were thawed and resuspended in a $\mathrm{pH} 8$ phosphate buffer containing EDTA and a protease inhibitor cocktail. The suspension was lysed using lysozyme and mechanical 
sonication. The resulting slurry was centrifuged at 3500 RPM to pellet cellular debris. The solution containing the protein of interest was decanted and diluted 1:1 into the same phosphate buffer. Proteins were purified using a Biorad Profinity $5 \mathrm{~mL}$ GST affinity column by FPLC (Biorad Prologic). Fractions containing protein of interest were dialyzed against a Tris- $\mathrm{NaCl}$ solution overnight. GST was cleaved from htt exon 1 using Factor Xa (Thermo). Overall protein concentration was determined by a Bradford assay. The final working concentration of htt exon 1 was adjusted to $20 \mu \mathrm{M}$ in a Tris- $\mathrm{NaCl}$ buffer.

\subsubsection{Atomic Force Microscopy.}

The purified GST protein and co-incubated peptides were maintained at $37^{\circ} \mathrm{C}$ in Eppendorf tubes and $1400 \mathrm{rpm}$ for the duration of the experiment in an orbital mixer. At $24 \mathrm{~h}$ after cleavage of GST with factor Xa protease, a $5.0 \mu \mathrm{L}$ aliquot of each protein incubation was spotted onto a freshly cleaved mica substrate (Ted Pella Inc., Redding, CA), for 30 seconds, washed with $200 \mu$ l of ultra-pure water to remove salt and dried gently with a stream of clean air. The mica was placed on metal pucks and stored in a covered petri dish until imaging.

AFM imaging Experiments were performed with a Nanoscope $V$ MultiMode scanning probe microscope (Veeco, Santa Barbara,CA) equipped with a closed loop vertical engage J-scanner. All images were acquired with diving board shaped silicon-oxide cantilevers with a nominal spring constant of $40 \mathrm{~N} / \mathrm{m}$ and resonance frequency of about $\sim 300 \mathrm{kHz}$. Scan rates were set at $1-2 \mathrm{~Hz}$ with cantilever drive frequencies $10 \%$ below resonance. 


\subsection{Results and Discussion}

4.3.1 Nt17 and polyP form two stable heterodimeric complex conformers.

Nt17 and polyP were incubated together in an ammonium acetate buffer at a $\mathrm{pH}$ of approximately 7.0 . The mass spectrum was dominated by the $[\mathrm{M}+2 \mathrm{H}]^{2+} \mathrm{Nt} 17$ monomer, as well as the PolyP monomer of the same charge; however, several dimeric species were also present, including but not limited to, the Nt17 homodimer $\left([\mathrm{M}+3 \mathrm{H}]^{3+}\right)$ and a Nt17-PolyP heterodimer $\left([\mathrm{M}+3 \mathrm{H}]^{3+}\right)$. Other multimers, such as the Nt17 homotrimer and the homotetramer were observed, but did not decrease in abundance in the presence of the PolyP peptide. Additionally, a PolyP homomultimer was not observed, nor was any evidence of higher charge heteromultimers (e.g., Nt17-PolyP $[\mathrm{M}+4 \mathrm{H}]^{4+}$ ) observed. Only the heterodimer species was observed. All collision cross sections can be found in Table 1.

Figure 4.1 shows expanded regions of representative IMS-MS distributions of a co-incubated Nt17-polyP sample. Figure 1A depicts the structural heterogeneity of the Nt17-PolyP heterodimer. Two distinct multimer structures that persist in the gas phase $(1002.4 \mathrm{~m} / \mathrm{z})$ are evident in the distribution. The origin of these complexes could stem from non-helical (compact, shorter drift time) and helical (elongated, longer drift time) association of Nt17 with PolyP, or PolyP association with the Nt17 helix via two separate faces. The more compact conformer is roughly $20 \%$ more abundant than its more elongated counterpart. The first conformer has a peak centered at $9.0 \mathrm{~ms}$, with a peak width 
of $0.2 \mathrm{~ms}$ (FWHM). This arrival time corresponds to a collision cross section of $539 \pm 1 \AA^{2}$.
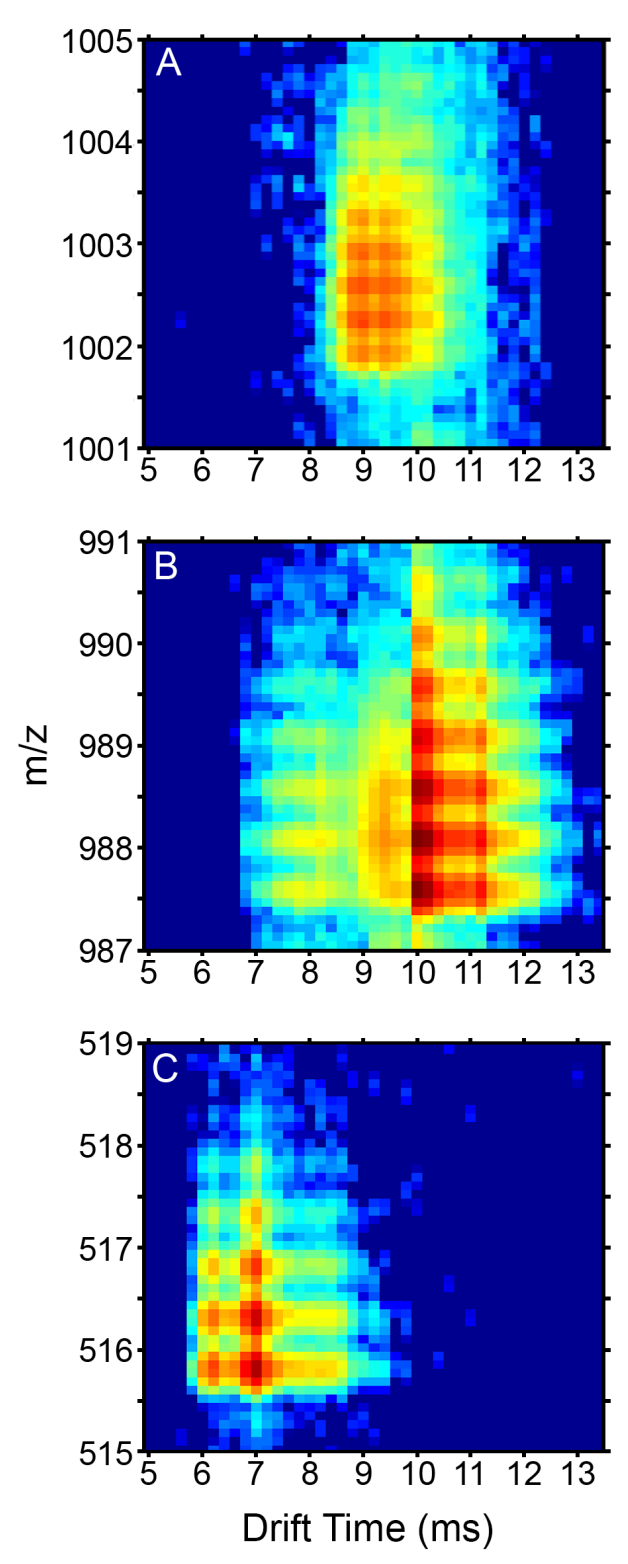

Figure 4.1. Expanded Regions of Representative IMS-MS distributions. A, distribution of the Nt17-PolyP $[\mathrm{M}+3 \mathrm{H}]^{3+}$ heterodimers. $\mathrm{B}$, distribution of the $[\mathrm{M}+2 \mathrm{H}]^{2+} \mathrm{Nt17}$ monomer. $\mathrm{C}$, distribution of the $[\mathrm{M}+2 \mathrm{H}]^{2+}$ PolyP monomer. Each panel is shown over the entire drift time range.
Though distinct, the peak is not fully resolved from the more elongated conformer. It is difficult to determine if a third structure is present in the $t_{D}$ space between the two conformations due to significant overlap; however later deuterium exchange experiments suggest that these are the only two conformer types of this species (see Figure 3). The second, more elongated conformation had an arrival time centered at $9.4 \mathrm{~ms}$, with a calculated cross section of $565 \pm 1 \AA^{2}$. Each conformation was drift-selected in the IMS drift tube, then mass selected in the mass analyzer before being subjected to increasing normalized collision energy. In this manner, we determined that the two conformations do not differ significantly in complex strength (Figure 2B); each conformer required approximately 15 
normalized collision energy (NCE) for $50 \%$ signal decay. This may suggest that the two conformers are bound similarly, perhaps including the same amino acid residues.

Several conformations of monomeric Nt17 and PolyP were apparent from the same mass spectrometry experiments in which the heterodimeric conformers were observed (Figure 1B and C). MDS suggests that the two most abundant Nt17 conformations can be represented by a semi-helical random structure (10.0 $\mathrm{ms}$ ) and an elongated, mostly helical structure (11.2 ms). These conformations are in good agreement with previous reports that indicate structural heterogeneity in the gas phase (see Chapter 3). The other two monomeric structures $(8.0 \mathrm{~ms}$ and $9.4 \mathrm{~ms}$ ) are dissociation products from higher order multimers. For example, the conformer arriving at $9.4 \mathrm{~ms}$ is most likely due to dissociation of the Nt17PolyP heterodimer complex at the same drift time. Analysis of the isotopic envelope indicated that no multimers with number of monomer units/charge $(n / z)$ $=1 / 2\left(\right.$ e.g., $[2 \mathrm{M}+4 \mathrm{H}]^{4+}$, which would have the same $\mathrm{m} / \mathrm{z}$ as $\left.[\mathrm{M}+2 \mathrm{H}]^{2+}\right)$ were present in the sample. The more elongated conformer is roughly one-tenth the abundance of the more compact structure, indicating that solution structure giving rise to the more compact structure is the dominant motif. The presence of both conformations suggests an equilibrium between species. The same argument can be applied to the PolyP peptide. Figure $1 \mathrm{C}$ shows the drift distribution of the PolyP $[\mathrm{M}+2 \mathrm{H}]^{2+}$ peptide. Both observed conformers are on the same cross section order as other gas-phase PolyP peptides of similar length, which were identified as mostly PPII helices with one or two residues exhibiting 
residual cis PPI structure. ${ }^{19}$ The first, most compact, conformation has a drift time of $6.2 \mathrm{~ms}$. The second, more elongated conformer has a drift time of $7.0 \mathrm{~ms}$, which corresponds to a gas phase cross section of $276 \pm 3 \AA^{2}$. As with the Nt17 monomer, no evidence of any isobaric homomultimeric species was observed.

\begin{tabular}{c|c|c|c} 
Species $^{a}$ & Charge State $^{b}$ & Drift Time $(\mathrm{ms})^{c}$ & Cross Section $\left(\AA^{2}\right)^{d}$ \\
\hline Nt17 & {$[\mathrm{M}+2 \mathrm{H}]^{2+}$} & 10.0 & $401 \pm 1$ \\
& & 11.2 & $443 \pm 2$ \\
PolyP & {$[\mathrm{M}+2 \mathrm{H}]^{2+}$} & 7.0 & $276 \pm 3$ \\
Nt17-PolyP & {$[\mathrm{M}+3 \mathrm{H}]^{3+}$} & 9.0 & $539 \pm 1$ \\
& 9.4 & $565 \pm 1$
\end{tabular}

Table 4.1. Calculated Collision Cross Sections for All Relevant Species. ${ }^{a}$ Species name as it is referred to in the body of the manuscript and in Figure $1 .{ }^{b}$ Charge state determined by peak spacing in isotopic envelope for drift-selected species. ${ }^{c}$ Drift time determined by peak center in the IMS-MS distribution. See Figure 1 for IMS-MS heat map. ${ }^{d}$ Gas-phase collision cross section determined by Equations 1 and 2 using experimental values.

Two Nt17-PolyP heterodimer conformations were observed. In an attempt to discern details beyond gas-phase cross section, each conformer was mobility selected using the ion mobility separation, then subsequently mass-selected and fragmented in the mass spectrometer. The abundance of the parent ion was monitored as a function of normalized collision energy. It should be noted that in this study, we used helium for the collision gas in the linear ion trap. Typically, a less polarizable gas, such as argon, is used for such studies if thermodynamics are to be directly calculated; ${ }^{25,}{ }^{26}$ however, in this case, only a relative 


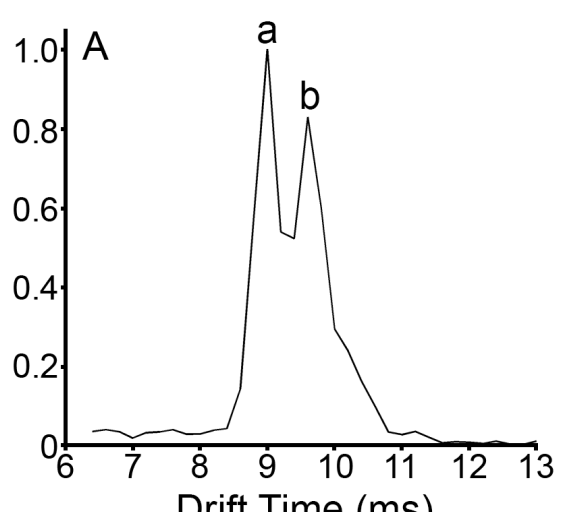

Drift Time (ms)
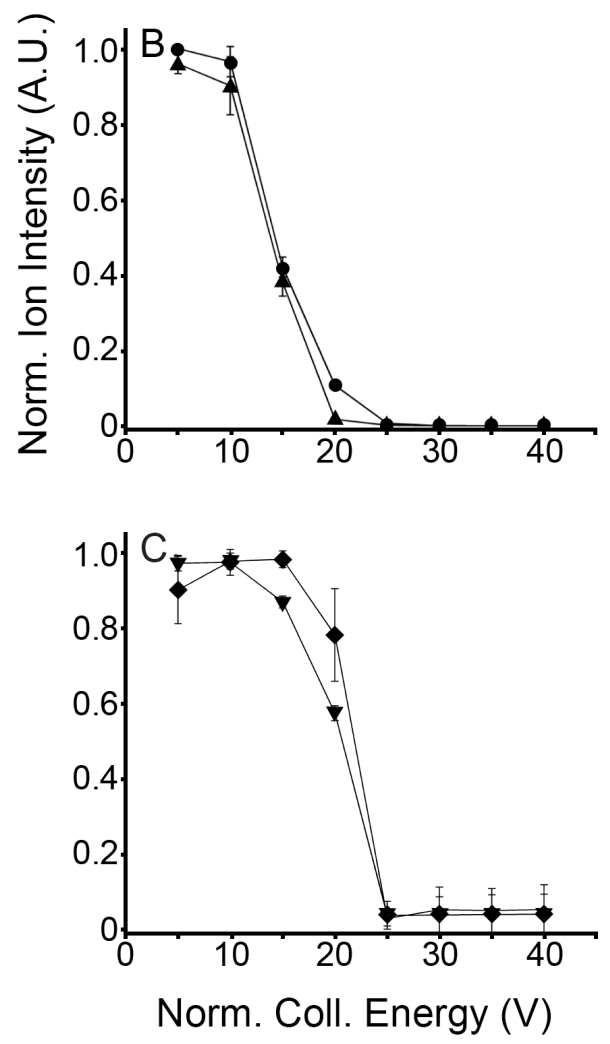

Figure 4.2. Nt17-PolyP heterodimer energetics. $A$, arrival time distribution of the heterodimer species, with labeled conformers 'a' (9.0 ms) and 'b' (9.4 ms). B, normalized ion signal as a function of normalized collision energy for each heterodimer conformer. Circles: conformer 'a'. Triangles: conformer ' $b$ '. C normalized ion signal as a function of normalized collision energy for two elongated Nt17 homodimer conformers. comparison of strength is necessary.

Thus, the use of helium gas is

acceptable for this study.

Figure 4.2A and $\mathrm{B}$ show the extracted drift time distribution of the Nt17-polyP heterodimer and the relative strength of each conformer.

The conformers do not differ in dissociation energy; both conformers have a $50 \%$ signal decay occurring at roughly $15 \mathrm{NCE}$, which would indicate that both complexes are energetically similar (Figue 4.2B).

Nt17 homodimer dissociation (Figure 4.2C) shows dissociation at approximately 20 NCE. Interestingly, the more elongated $\mathrm{Nt} 17$ homodimer conformer (filled diamonds, Figure

4.2C) forms a slightly stronger complex than the more compact conformer (filled squares, Figure 4.2C). In general, however, both Nt17-polyP heterodimers are not as 
energetically stable as the Nt17 homodimers. It should be noted that from these experiments, it is impossible to tell where exactly intermolecular interactions occur for the peptide multimers. The energetics of association can have implication in normal function of the huntingtin protein overall. If the polyP region does, in fact, regulate the function of Nt17 as suggested by Truant and coworkers, ${ }^{1}$ then one would expect a relatively weak interaction such that Nt17 can undergo its normal function. Additionally, the relative strength of the homodimeric Nt17 complex suggests a greater affinity for itself over a small molecule binding partner, which is not surprising considering its potential role in aggregation. . $^{3,9}$, 27

\subsubsection{Heterodimer conformations vary in deuterium uptake.}

Following the evidence of two heterodimeric species, gas-phase hydrogen-deuterium exchange (HDX) was performed to determine differences in deuterium uptake by conformer. By extension, deuterium uptake may help determine sites of binding and provide insight into the location of exchange sites in the proximity of charge sites. An explanation of the gas-phase HDX mechanism can be found in Chapter 1, Section 1.6.3.

Figure 4.3 shows IMS-MS distributions for the Nt17-polyP heterodimer (panel A), the Nt17 monomer (panel B), and the polyP monomer (panel C). Each of these species are the same as in Figure 4.1A - C. Overall, very little deviation in drift time was observed for all species; a $0.2 \mathrm{~ms}$ shift to higher drift times was 

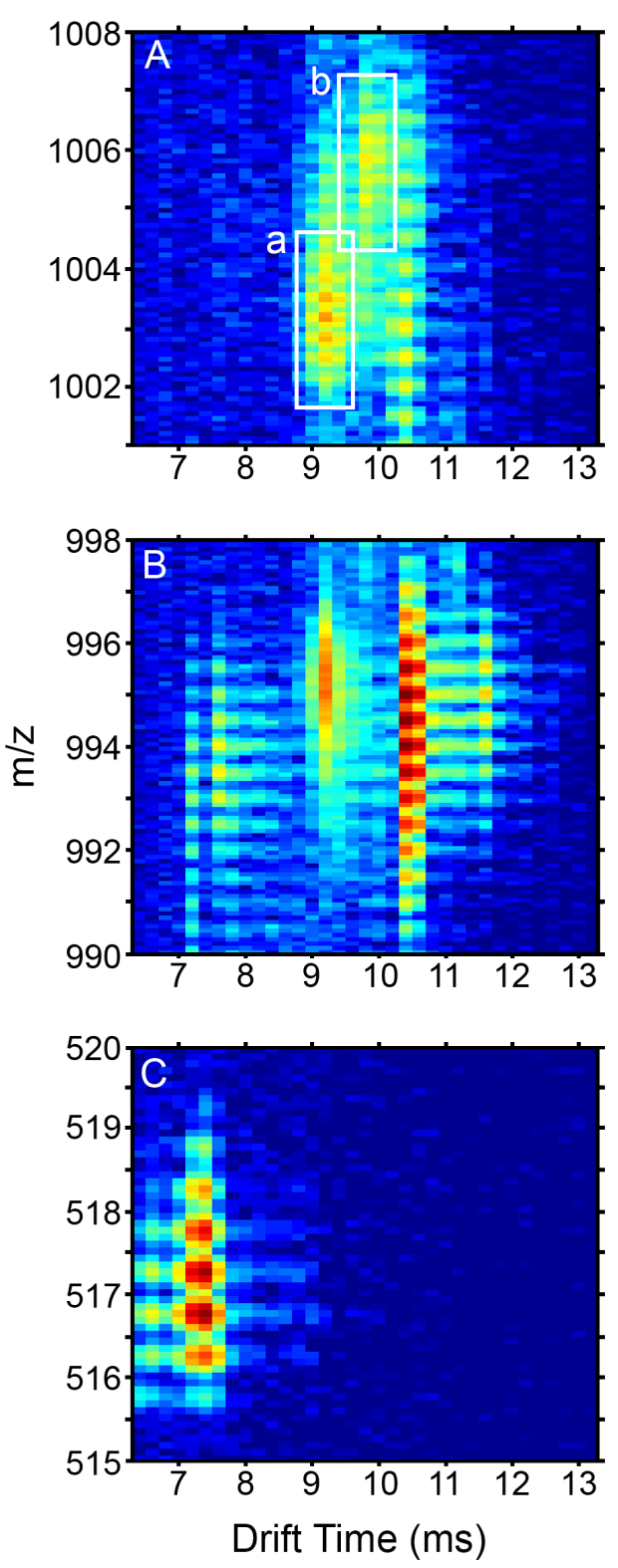

Figure 4.3. Deuterated IMS-MS

distributions. $A$, deuterated $[\mathrm{M}+2 \mathrm{H}]^{2+}$ Nt17-PolyP heterodimer complex.

Conformers ' $a$ ' and ' $b$ ' are labeled the same as they are in Figure 2. $B$, deuterated $[\mathrm{M}+2 \mathrm{H}]^{2+} \mathrm{Nt17}$ monomer. C, deuterated $[\mathrm{M}+2 \mathrm{H}]^{2+}$ PolyP monomer. Each panel is shown over the entire drift range. observed, which is expected when

using a heterogeneous drift gas

mixture of larger gas size and mass. ${ }^{28}$

A surprising result is the

differential deuterium uptake

exhibited by the two Nt17-PolyP

heterodimer conformations (Figure

4.3A and Figure 4.4). The more

compact conformation (conformer 'a')

shifted upward in $\mathrm{m} / \mathrm{z}$ by 1.6 , which

leads to an average uptake of $4 \pm 2$

deuterium (see Figure 4 for total

deuterium uptake). Conversely,

conformer 'b' incorporated an

average of $10.8 \pm 0.5$ deuterium, over

twice the uptake of conformer 'a'.

Both Nt17 monomer conformations

exhibited the same uptake of

approximately 13 deuterium atoms,

which is higher than that of the

complex, indicating several

hydrophilic residues may be involved in binding. Finally, the PolyP peptide only

exhibited an uptake of 2 deuterium atoms. Since PolyP does not contain 


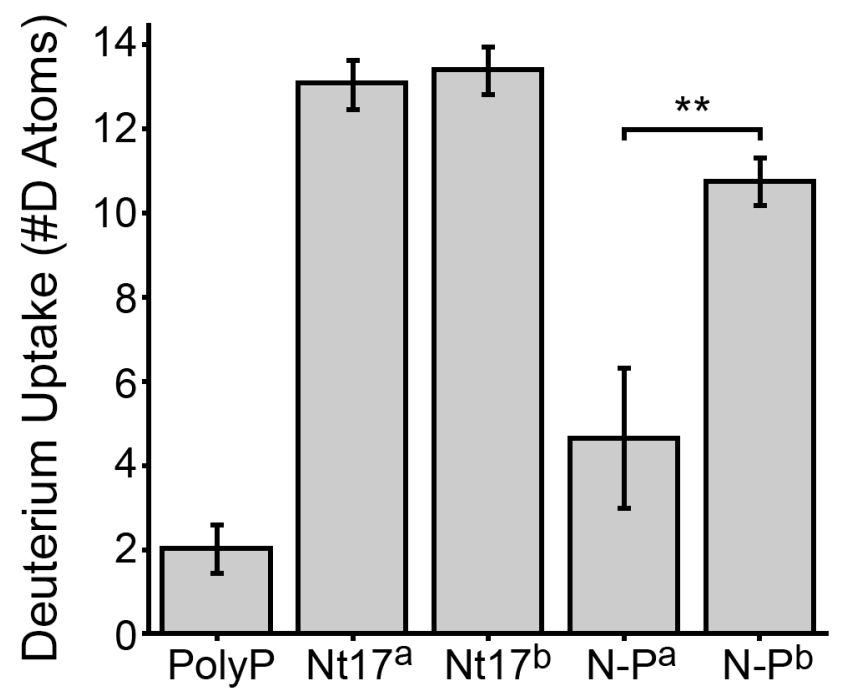

Figure 4.4. Deuterium uptake by species. Calculated deuterium uptake for species highlighted in Figure 3. PolyP: polyproline peptide $[\mathrm{M}+\mathrm{H}]^{2+} . \mathrm{Nt17^{a }}$ and $\mathrm{Nt17^{b }}$ : Nt17 peptide $[\mathrm{M}+2 \mathrm{H}]^{2+}$ at 10.6 and $11.6 \mathrm{~ms}$, respectively. $N-P^{a}$ and $N-P^{b}$ : $\mathrm{Nt17-PolyP}$ heterodimer complex $[\mathrm{M}+3 \mathrm{H}]^{3+}$ at 9.2 and $9.8 \mathrm{~ms}$, respectively. Two asterisks denotes highly significant statistical difference $(p<0.01)$ exchangeable backbone amide hydrogens, these two deuterium atoms found in the PolyP tract must result from charge site deuteration; ${ }^{29,30}$ once the deuteron is transferred to the charge site, it cannot be redistributed as it is in peptides containing other labile heteroatom sites. The disparate uptake data may indicate that polyP can associate with multiple faces

of Nt17, perhaps via similar interactions; however, one site provides more access to labile heteroatoms than does the other. Conversely, polyP could be associated in roughly the same place in both structures, but Nt17 could sample several conformations. Serines 13 and 16 have been implicated in Nt17-polyP interactions. ${ }^{1}$ Both S13E/S16E mutations and Nt17 hyperphosphorylation (M8P) by IKK kinase inhibition resulted in reduced Nt17-polyP interaction for pathogenic and non-pathogenic htt exon $1 .{ }^{1}$ When taken with the HDX data reported here, it seems the more compact conformation, which has less deuterium incorporation than its more elongated counterpart, could include a S13/16-polyP interaction. 


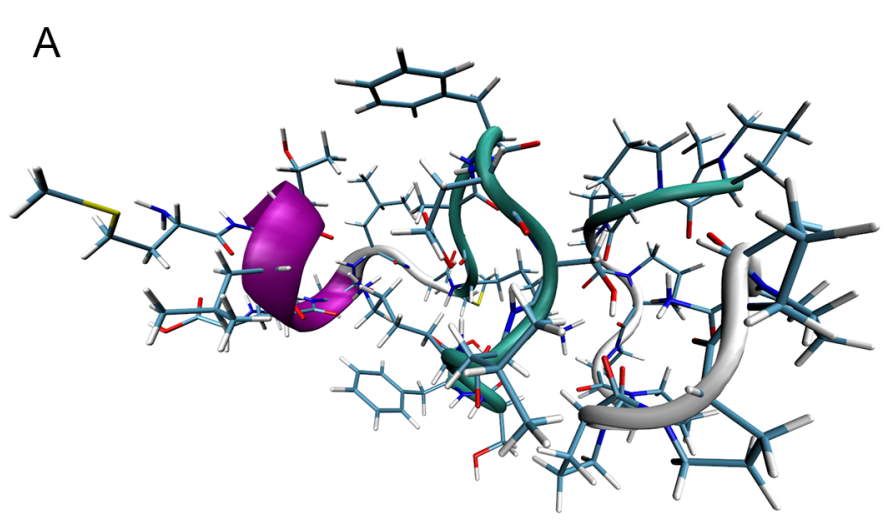

B

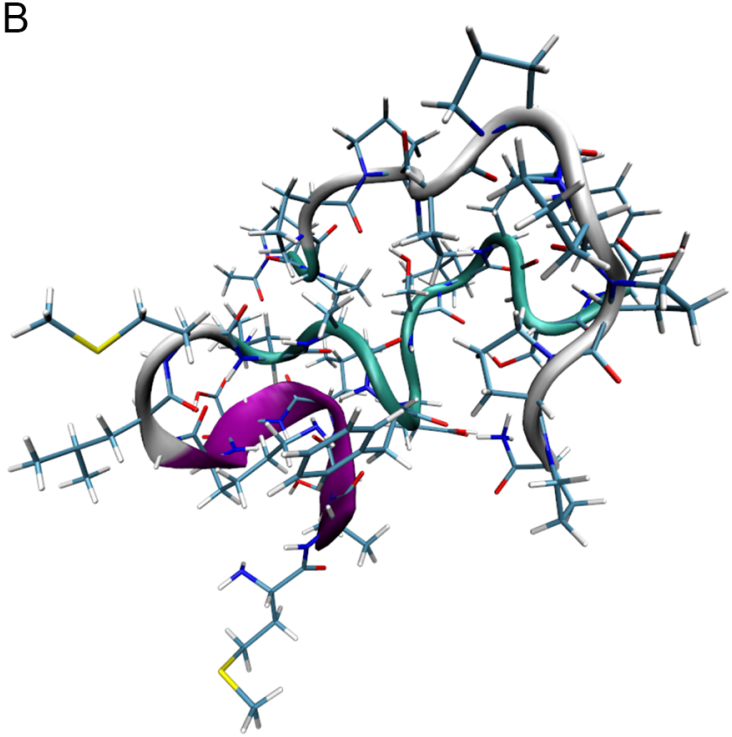

Figure 4.5. Nt17-polyP heterodimer gas-phase structures. A. Compact conformer, labeled 'a' in Figures 4.1 - 4.4. B. Elongated conformer, labeled 'b' in Figures 4.1 - 4.4 .
4.3.3 Gas-phase Nt17-polyP

heterodimer structure.

Figure 4.5 shows the

lowest energy conformations

within $\pm 1 \%$ of the measured

CCS (found in Table 4.1) from

MDS. Panel A represents a

compact conformer ('a' in

Figures $4.1-4.4)$, and panel B represents a more elongated conformer ('b' in Figures 4.1 4.4). Both conformers exhibit a surprising lack of extended $\alpha$ helicity in Nt17. The polyP constituent in the compact conformer associates with Nt17 mostly by the latter half (M8 - S16) of the peptide. While there is some small degree of helicity from residues $\mathrm{A} 2$ to $\mathrm{E} 5$, the extended helix that was prevalent in the homodimeric complex, as well as monomeric Nt17 was not evident. Hydrophilic residues are buried either in intramolecular association (K6) or buried in the interaction with the polyP tract $(\mathrm{K} 9, \mathrm{~K} 15)$. The inaccessibility of these residues would explain the 
lack of deuterium uptake in conformer 'a' (see Figure 4.3 and 4.4). Since the charges are either buried or otherwise

inaccessible to collisions with deuterium which initiate the relay exchange mechanism, deuterium exchange cannot efficiently proceed. The charged polyP C-terminus self-solvates and thus, is not highly accessible (right portion of panel A, white backbone region), thus not interacting with Nt17. This is an important point, because in a htt exon 1 context, the PPII helix would not be charged.

Figure 4.5B shows the more elongated conformer. In contrast to Figure 4.5A, this conformation shows a more extended Nt17 constituent. In this model, hydrophilic residues $\mathrm{K} 6$ and $\mathrm{K} 9$ are solvent-exposed, which would lead to a greater deuterium uptake, as shown in Figures 4.3 and 4.4. Additionally, residue M8 on Nt17 was pointed away from the polyP tract, as opposed to potentially interacting with polyP in the more compact state. The same helical content was observed in the elongated conformer as was in the compact conformer, though the C-terminal portion of Nt17 did not turn back on itself, as was evident in the compact conformation. Lastly, it appears that in this arrangement, the polyP tract is oriented almost around $\mathrm{Nt17}$, with hydrogens on $\mathrm{S} 13$ and $\mathrm{S} 16$ within $\mathrm{H}$-bond distance of a backbone carbonyl on polyP. To verify the structures, HAS and surface accessability were incorporated into exchange kinetics and deuterium uptake was calculated for each candidate structure. The most compact candidate structure was indeed calculated to exchange on average 4.5 deuterium atoms, while the more elongated candidate structure should exchange roughly 9.2 deuterium atoms. These calculated values are in good agreement with actual 
gas-phase deuterium uptake measurements, thus, these are highly plausible gas-phase structures. It must be stressed here that these are gas-phase structures, and may be reminiscent of condensed-phase structure. These are structures that most closely represent the measured CCS and deuterium uptake patterns; however, that is not to say that the gas-phase conformations shown here and in other works ${ }^{19,31-35}$ are completely erroneous. On the contrary, these are gas-phase structures that are reminiscent of solution-phase conformations that retain structural heterogeneity when transitioning into the gas-phase.

Two striking similarities appear upon inspection of the structures reported in Figure $4.5 \mathrm{~A}$ and $\mathrm{B}$. First is the lack of the extended helical conformation in Nt17. In solution, the Nt17 tract, both in the context of polyQ and without, adopts a helical conformation when in proximity of a binding partner: either another Nt17 tract $^{4,14}$ or a lipid bilayer. ${ }^{12,36,37}$ Additionally, the helical association could be the driving force for oligomerization in htt exon $1 .{ }^{4,5}$ The lack of extended helix observed here could have implications in intrinsic polyP regulation of Nt17. ${ }^{1}$ The polyP region regulates $\mathrm{Nt} 17$ by associating with the $\mathrm{C}$-terminal portion of the tract, part of which may be helical in amyloid fibrils, ${ }^{4,14,15}$ thus, not allowing helical formation of a full, extended a-helix ranging from A2 to E12. This would explain the inherent lack of helicity in $\mathrm{Nt} 17-\mathrm{Q}_{\mathrm{n}}-\mathrm{P}_{\mathrm{m}}$ and htt exon 1 monomeric structures measured by spectroscopy, ${ }^{4}$ while Nt17 alone in its monomeric and multimeric form is free to form extended $\alpha$-helices. This could also explain the acceleration of aggregation observed when $\mathrm{Nt17}$ is associated to extended polyQ regions without the proline-rich domain; $;^{9,10}$ the proline domain is not present to 
regulate Nt17 helical formation, thus allowing Nt17 to drive aggregation through helical association.

The second similarity in the two structures are the involvement, or relative proximity, of S13 and S16 to the polyP tract. CYP-labeled Phosphomimetic and hyperphosphorylated htt exon 1 , with $Q_{n}=9$ showed a dramatic reduction in FRET intensity compared with wild type htt exon 1. In the gas-phase structures reported in the current work, phosphorylation of S13 and S16 could feasibly disrupt interaction with the polyP sequence. In previous work, htt exon 1 with $\mathrm{S}$ to D mutation showed a decrease in aggregation kinetics and decreased aggregate stability. Phosphorylation of these $\mathrm{S}$ residues both reduces polyP interaction in native htt exon 1 , and suppresses aggregation kinetics. ${ }^{7}{ }^{16}$ While these mutations were not probed in this study, it is worth pointing out a similarity between the gas phase structures reported here and the results observed in other work.

\subsubsection{Incubation with polyP reduces htt exon 1 fibril formation.}

Figure 6 shows representative AFM images taken at 24 hours after GST cleavage. Panel A depicts htt exon 1, while panels B and C show htt exon 1 coincubated with Nt17 and polyP, respectively (see Experimental Procedures for incubation conditions). Htt exon 1 formed characteristic amyloid fibrils. The structures shown in panel A are nearly identical in height and length to those reported elsewhere using the same protein. When co-incubated with Nt17, htt exon 1 still forms amyloid fibrils. Previous experiments that reported co- 
incubation of htt exon 1 peptides with Nt17 showed similar morphologies to those reported in Figure 6B at the 24 hour time point. In their study, Mishra et al. report more stable oligomer structures when htt exon 1 is co-incubated with $\mathrm{Nt17}$ at shorter time points. ${ }^{5}$ Figure $6 \mathrm{C}$ shows a representative AFM image taken at $\mathrm{t}=$ 24 hours of htt exon 1 co-incubated with the polyP peptide. A striking difference is observed relative to Figure $6 \mathrm{~A}$; significantly fewer extended fibrillar species are apparent in the sample co-incubated with polyP. Short, amorphous, possibly oligomeric species are observed, much like Mishra et al. observe for their Nt17htt exon 1 co-incubation at shorter time points. ${ }^{5}$ It would appear, then, that the polyP peptide has an amyloidogenic inhibitory effect in a model protein system.
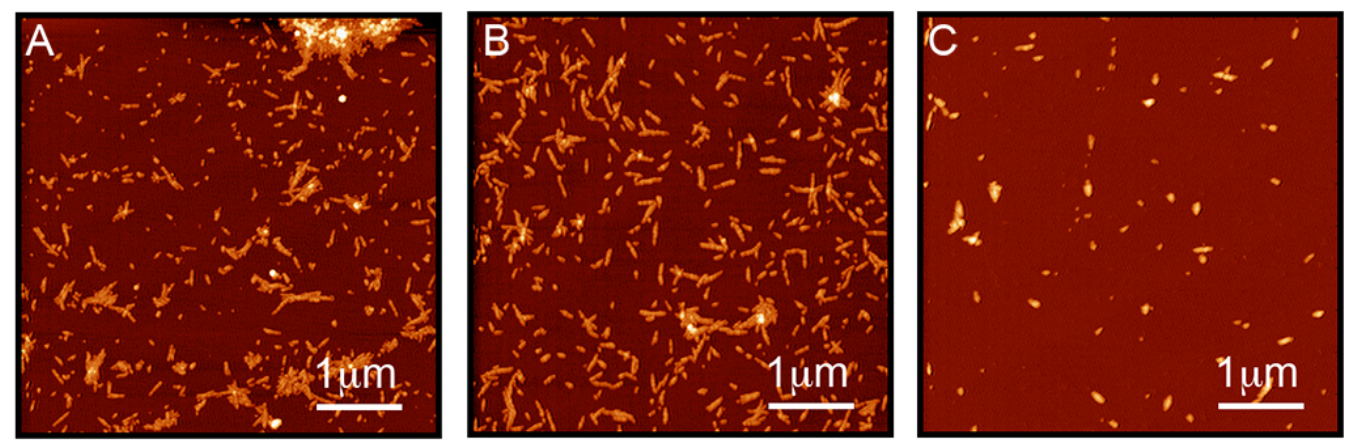

Figure 4.6. Representative AFM images. Images of htt exon 1 (51Q, $t=24$ hrs.) deposited on a mica surface. A htt exon 1. B htt exon 1 co-incubated with Nt17. C htt exon 1 incubated with polyP.

Several interpretations of this observed polyP inhibitory effect could be plausible. First, the polyP peptide could interact with the polyQ domain, which could stabilize the random nature of polyQ and prohibit the transition to $\beta$-sheet. Additionally, interaction at multiple sites in the polyQ domain could increase the distance between adjacent polyQ chains. PolyQ segments could transiently 
sample PPII conformations, ${ }^{20,38}$ which would be amenable for intermolecular interaction with the polyP peptide in its PPII state. Previous studies have shown inhibition of short polyQ fragments in a PPII conformation by N-methylated short peptide sequences. ${ }^{39}$ Since the IMS-MS data reported in this study did not take into account a polyQ tract, no definitive comment can be made on the inhibition of polyQ aggregation by direct intermolecular interaction with polyP. Although the addition of the polyP peptide could interact with the proline-rich region in htt exon 1 and stabilize the PPII character further, in the IMS-MS experiments we do not observe a polyP homomultimer of any kind, which suggests that this interaction could be unlikely. Still, because the full proline-rich region was not sampled in IMS-MS, a C-terminal interaction responsible for aggregation suppression cannot be ruled out. A comment can be made, however, on inhibition of htt exon 1 aggregation through a process mediated by Nt17. Like previous studies that have shown aggregation inhibition through targeting Nt17 with small peptides, ${ }^{5}$ and molecular chaperones ${ }^{40,41}$ polyP could associate with $\mathrm{Nt17}$ at a critical interface causing stabilization of an oligomeric state by not allowing further polyQ association. Interstitial insertion of polyP into the $\mathrm{N}$-terminal htt exon 1 helical bundles could act in the same manner as Nt17, increasing distance between polyQ chains and thus, increasing the energy required to form $\beta$-sheet rich aggregates. Alternatively, the polyP region could partially shut down Nt17 helix formation in disease-length htt exon 1 constructs, thus decreasing kinetics of fibril formation, though still proceeding through an oligomeric intermediate. While the polyQ-associated polyP region would no longer be in proximity of $\mathrm{Nt17}$ for 
regulation, free polyP peptide can insert and act to regulate $\mathrm{Nt17}$ in the same manner. In any case, incubation of htt exon 1 with a polyP peptide reduced fibril formation. Future studies are necessary to fully characterize this interaction.

\subsubsection{Interpretation of structural findings.}

This study reports, for the first time, multiple conformations of a Nt17polyP complex that could have implications in Huntington's disease-related amyloid formation. Previous work suggests that the C-terminal polyP region acts as a stabilizer of $\mathrm{Nt17}$ for short polyQ lengths; however, as polyQ length increases the resulting rigidity increases the distance between the Nt17 tract and the polyP tract. The increased distance allows Nt17 to participate in intermolecular associations with other Nt17 tracts, or perhaps the associated polyQ, to initiate fibril formation. This is supported by the data in Figure 4.2, where increased complexation energy is suggested for the Nt17 homodimer relative to the Nt17-polyP heterodimer; the increased energy supports the claim that Nt17 has a high self-affinity, and thus can act to nucleate aggregation. Additionally, no decrease in Nt17 homomultimer is observed upon addition of the polyP peptide, which, again indicates that the Nt17 helix-helix interaction overcomes the Nt17-polyP hetrodimerization. This could have implications for the native function of $\mathrm{Nt17}$ as a nuclear export signal and modification target, ${ }^{27,42,43}$ as complexation with polyP near an active site would act to regulate kinase activity that leads to nuclear export signaling, especially if key hydrophilic residues are buried in the complex. 
The gas-phase structures reported in Figure 4.5 provide a clearer picture of what potential Nt17-polyP complexes may resemble. While these are gasphase conformations, it should be noted that the method used here has been used in previous experiments to accurately predict condensed phase structure and pinpoint protein heterogeneity. ${ }^{32,35,44}$ Along with these studies, the work may indicate that condensed phase Nt17 structure could range anywhere from mostly disordered ${ }^{4}$ to fully extended. ${ }^{45}$ Indeed, previous IMS-MS experiments report that Nt17 could consist of a heterogenous mix of partial helix and extended helix in the gas phase (see Chapter 3). Extended Nt17 homodimers could consist of two elongated antiparallel helices. These data lead to the hypothesis that Nt17 only adopts a fully extended helical structure when involved in a complex. In both the compact and elongated structures shown in Figure 4.5, however, Nt17 adopts a mostly random coil motif. The differential deuterium uptake by conformation is then explained by the involvement of hydrophilic residues in stabilizing the complex and the relative proximity to charge sites, and to a much lesser extent, the presence of secondary structure. Based on these data, it could be hypothesized that C-terminal Nt17 complexation with the polyP helix stabilizes the random coil nature of $\mathrm{Nt} 17$, thus preventing a transition to the extended $\alpha-$ helix, as observed in amyloid fibrils ${ }^{14,15}$ and hypothesized for an extended homodimer. In addition to inhibition through insertion, the co-incubated polyP could possibly act to disallow the helical transition in Nt17, thus promoting formation of more amorphous aggregates, as shown in Figure 4.6, or entirely abolishing aggregation. A caveat to this work as with the work presented in 
Chapter 3 , is the lack of a poly $Q$ sequence. The poly $Q$ linker would restrict the freedom of the polyP and Nt17 domains and may impart structure into these regions; however, solid-state NMR results indicate that in a mature fibril, the polyP PPII helix abruptly stops immediately before the polyQ domain and the $\beta$ sheet character of the polyQ region only extends to the last two residues of $\mathrm{Nt} 17 .^{15}$

The work described in this chapter highlights potential interactions between $\mathrm{Nt} 17$ and the polyP regions on htt exon 1. PolyP could serve as a regulator of Nt17 in non-pathogenic htt exon 1. Two Nt17-polyP heterodimer complexes were observed in the gas phase, both of which are energetically weaker than an Nt17 homodimer. A gas-phase deuterium exchange experiment shows differential uptake between the conformations, which suggested greater availability of charge sites in Nt17, which could have implication in complex orientation, presence of secondary structure, or both. Simulated annealing experiments reveal a mostly unstructured, C-terminal Nt17 region in the gas phase when complexed to the all-trans polyP peptide, which is in stark contrast to gas-phase orientation of the Nt17 homodimeric complex, which, in its most elongated state, is composed of two extended $\alpha$-helices arranged antiparallel to each other (see Chapter 3). Incubation of disease-length htt exon 1 with the polyP tract led to a reduction in fibril formation relative to control htt exon 1, which suggests that polyP has an inhibitory effect on htt exon 1 . Together, the data described in this work suggest that the polyP region inhibits formation of the amphipathic $\alpha$-helix by $\mathrm{Nt17}$, thus reducing the ability to participate in 
energetically favorable helix-helix interactions. This work has laid the groundwork for future studies seeking not just blockage of a critical residue, but perhaps blockage of a critical secondary structure for HD remediation. 


\subsection{References}

(1) Caron, N. S., Desmond, C. R., Xia, J., and Truant, R. (2013)

Polyglutamine domain flexibility mediates the proximity between flanking sequences in huntingtin, Proceedings of the National Academy of Sciences 110, 14610-14615.

(2) Burke, K. A., Kauffman, K. J., Umbaugh, C. S., Frey, S. L., and Legleiter, J. (2013) The Interaction of Polyglutamine Peptides With Lipid Membranes is Regulated by Flanking Sequences Associated with Huntingtin, Journal of Biological Chemistry.

(3) Jayaraman, M., Mishra, R., Kodali, R., Thakur, A. K., Koharudin, L. M. I., Gronenborn, A. M., and Wetzel, R. (2012) Kinetically Competing Huntingtin Aggregation Pathways Control Amyloid Polymorphism and Properties, Biochemistry 51, 2706-2716.

(4) Jayaraman, M., Kodali, R., Sahoo, B., Thakur, A. K., Mayasundari, A., Mishra, R., Peterson, C. B., and Wetzel, R. (2012) Slow Amyloid Nucleation via alpha-Helix-Rich Oligomeric Intermediates in Short Polyglutamine-Containing Huntingtin Fragments, Journal of Molecular Biology 415, 881-899.

(5) Mishra, R., Jayaraman, M., Roland, B. P., Landrum, E., Fullam, T., Kodali, R., Thakur, A. K., Arduini, I., and Wetzel, R. (2012) Inhibiting the Nucleation of Amyloid Structure in a Huntingtin Fragment by Targeting alpha-Helix-Rich Oligomeric Intermediates, Journal of Molecular Biology 415, 900-917.

(6) O'Rourke, J. G., Gareau, J. R., Ochaba, J., Song, W., Rasko, T., Reverter, D., Lee, J., Monteys, A. M., Pallos, J., Mee, L., Vashishtha, M., Apostol, B. L., Nicholson, T. P., Illes, K., Zhu, Y. Z., Dasso, M., Bates, G. P., Difiglia, M., Davidson, B., Wanker, E. E., Marsh, J. L., Lima, C. D., Steffan, J. S., and Thompson, L. M. (2013) SUMO-2 and PIAS1 Modulate Insoluble Mutant Huntingtin Protein Accumulation, Cell Reports 4, 362-375.

(7) Mishra, R., Hoop, C. L., Kodali, R., Sahoo, B., van der Wel, P. C. A., and Wetzel, R. (2012) Serine Phosphorylation Suppresses Huntingtin Amyloid Accumulation by Altering Protein Aggregation Properties, Journal of Molecular Biology 424, 1-14.

(8) Aiken, C. T., Steffan, J. S., Guerrero, C. M., Khashwji, H., Lukacsovich, T., Simmons, D., Purcell, J. M., Menhaji, K., Zhu, Y. Z., Green, K., LaFerla, F., Huang, L., Thompson, L. M., and Marsh, J. L. (2009) Phosphorylation of Threonine 3 IMPLICATIONS FOR HUNTINGTIN AGGREGATION AND NEUROTOXICITY, Journal of Biological Chemistry 284, 29427-29436.

(9) Crick, S. L., Ruff, K. M., Garai, K., Frieden, C., and Pappu, R. V. (2013) Unmasking the roles of $\mathrm{N}$ - and $\mathrm{C}$-terminal flanking sequences from exon 1 of huntingtin as modulators of polyglutamine aggregation, Proceedings of the National Academy of Sciences 110, 20075-20080.

(10) Williamson, T. E., Vitalis, A., Crick, S. L., and Pappu, R. V. (2010) Modulation of Polyglutamine Conformations and Dimer Formation by the $\mathrm{N}-$ Terminus of Huntingtin, Journal of Molecular Biology 396, 1295-1309. 
(11) Kim, M. W., Chelliah, Y., Kim, S. W., Otwinowski, Z., and Bezprozvanny, I. (2009) Secondary Structure of Huntingtin Amino-Terminal Region, Structure 17, 1205-1212.

(12) Michalek, M., Salnikov, E. S., Werten, S., and Bechinger, B. (2013) Membrane Interactions of the Amphipathic Amino Terminus of Huntingtin, Biochemistry 52, 847-858.

(13) Michalek, M., Salnikov, E. S., and Bechinger, B. (2013) Structure and Topology of the Huntingtin 117 Membrane Anchor by†a†Combined Solution and Solid-State NMR Approach, Biophysical journal 105, 699710.

(14) Sivanandam, V. N., Jayaraman, M., Hoop, C. L., Kodali, R., Wetzel, R., and van der Wel, P. C. A. (2011) The Aggregation-Enhancing Huntingtin $\mathrm{N}$-Terminus Is Helical in Amyloid Fibrils, Journal of the American Chemical Society 133, 4558-4566.

(15) Hoop, C. L., Lin, H.-K., Kar, K., Hou, Z., Poirier, M. A., Wetzel, R., and van der Wel, P. C. A. (2014) Polyglutamine amyloid core boundaries and flanking domain dynamics in huntingtin fragment fibrils determined by solid-state NMR, Biochemistry.

(16) Gu, X. F., Greiner, E. R., Mishra, R., Kodali, R., Osmand, A., Finkbeiner, S., Steffan, J. S., Thompson, L. M., Wetzel, R., and Yang, X. W. (2009) Serines 13 and 16 Are Critical Determinants of Full-Length Human Mutant Huntingtin Induced Disease Pathogenesis in HD Mice, Neuron 64, 828840.

(17) Burke, K. A., Godbey, J., and Legleiter, J. (2011) Assessing mutant huntingtin fragment and polyglutamine aggregation by atomic force microscopy, Methods 53, 275-284.

(18) Donohoe, G. C., Maleki, H., Arndt, J. R., Khakinejad, M., Yi, J., McBride, C., Nurkiewicz, T. R., and Valentine, S. J. (2014) A New Ion MobilityLinear Ion Trap Instrument for Complex Mixture Analysis, Analytical Chemistry 86, 8121-8128.

(19) Shi, L., Holliday, A. E., Shi, H., Zhu, F., Ewing, M. A., Russell, D. H., and Clemmer, D. E. (2014) Characterizing Intermediates Along the Transition from Polyproline I to Polyproline II Using Ion Mobility Spectrometry-Mass Spectrometry, Journal of the American Chemical Society.

(20) Darnell, G., Orgel, J., Pahl, R., and Meredith, S. C. (2007) Flanking polyproline sequences inhibit beta-sheet structure in polyglutamine segments by inducing PPII-like helix structure, Journal of Molecular Biology 374, 688-704.

(21) Bhattacharyya, A., Thakur, A. K., Chellgren, V. M., Thiagarajan, G., Williams, A. D., Chellgren, B. W., Creamer, T. P., and Wetzel, R. (2006) Oligoproline effects on polyglutamine conformation and aggregation, Journal of Molecular Biology 355, 524-535.

(22) Khakinejad, M. K., S; Valentine, S. (2015) Gas-phase hydrogen-deuterium exchange labeling of select peptide ion conformer types: a per-residue kinetics analysis, Journal of The American Society for Mass Spectrometry In Press. 
(23) Donohoe, G., Khakinejad, M., and Valentine, S. (2015) Ion Mobility Spectrometry-Hydrogen Deuterium Exchange Mass Spectrometry of Anions: Part 1. Peptides to Proteins, Journal of The American Society for Mass Spectrometry 26, 564-576.

(24) Muchowski, P. J., Schaffar, G., Sittler, A., Wanker, E. E., Hayer-Hartl, M. K., and Hartl, F. U. (2000) Hsp70 and Hsp40 chaperones can inhibit selfassembly of polyglutamine proteins into amyloid-like fibrils, Proceedings of the National Academy of Sciences 97, 7841-7846.

(25) Armentrout, P. B. (2013) The Power of Accurate Energetics (or Thermochemistry: What is it Good for?), Journal of the American Society for Mass Spectrometry 24, 173-185.

(26) Rodgers, M. T., and Armentrout, P. B. (2004) A thermodynamic "vocabulary" for metal ion interactions in biological systems, Accounts of Chemical Research 37, 989-998.

(27) Zheng, Z. Q., Li, A. M., Holmes, B. B., Marasa, J. C., and Diamond, M. I. (2013) An N-terminal Nuclear Export Signal Regulates Trafficking and Aggregation of Huntingtin (Htt) Protein Exon 1, Journal of Biological Chemistry 288, 6063-6071.

(28) Khakinejad, M., Kondalaji, S. G., Maleki, H., Arndt, J. R., Donohoe, G. C., and Valentine, S. J. (2014) Combining lon Mobility Spectrometry with Hydrogen-Deuterium Exchange and Top-Down MS for Peptide Ion Structure Analysis, Journal of The American Society for Mass Spectrometry 25, 2103-2115.

(29) Wyttenbach, T., and Bowers, M. T. (1999) Gas phase conformations of biological molecules: the hydrogen/deuterium exchange mechanism, Journal of the American Society for Mass Spectrometry 10, 9-14.

(30) Campbell, S., Rodgers, M. T., Marzluff, E. M., and Beauchamp, J. L. (1995) Deuterium exchange reactions as a probe of biomolecule structure. Fundamental studies of cas phase $\mathrm{H} / \mathrm{D}$ exchange reactions of protonated glycine oligomers with D2O, CD3OD, CD3CO2D, and ND3, Journal of the American Chemical Society 117, 12840-12854.

(31) Bleiholder, C., Do, T. D., Wu, C., Economou, N. J., Bernstein, S. S., Buratto, S. K., Shea, J. E., and Bowers, M. T. (2013) Ion Mobility Spectrometry Reveals the Mechanism of Amyloid Formation of A beta(2535) and Its Modulation by Inhibitors at the Molecular Level:

Epigallocatechin Gallate and Scyllo-inositol, Journal of the American Chemical Society 135, 16926-16937.

(32) Wyttenbach, T., and Bowers, M. T. (2011) Structural Stability from Solution to the Gas Phase: Native Solution Structure of Ubiquitin Survives Analysis in a Solvent-Free lon Mobility-Mass Spectrometry Environment, Journal of Physical Chemistry B 115, 12266-12275.

(33) Bernstein, S. L., Dupuis, N. F., Lazo, N. D., Wyttenbach, T., Condron, M. M., Bitan, G., Teplow, D. B., Shea, J. E., Ruotolo, B. T., Robinson, C. V., and Bowers, M. T. (2009) Amyloid-beta protein oligomerization and the importance of tetramers and dodecamers in the aetiology of Alzheimer's disease, Nature Chemistry 1, 326-331. 
(34) Wyttenbach, T., von Helden, G., and Bowers, M. T. (1996) Gas-Phase Conformation of Biological Molecules: Bradykinin, Journal of the American Chemical Society 118, 8355-8364.

(35) Shi, H. L., Pierson, N. A., Valentine, S. J., and Clemmer, D. E. (2012) Conformation Types of Ubiquitin $\mathrm{M}+8 \mathrm{H}(8+)$ lons from Water:Methanol Solutions: Evidence for the $\mathrm{N}$ and $\mathrm{A}$ States in Aqueous Solution, Journal of Physical Chemistry B 116, 3344-3352.

(36) Côté, S., Wei, G., and Mousseau, N. (2014) Atomistic mechanisms of huntingtin $\mathrm{N}$-terminal fragment insertion on a phospholipid bilayer revealed by molecular dynamics simulations, Proteins: Structure, Function, and Bioinformatics, n/a-n/a.

(37) Nagarajan, A., Jawahery, S., and Matysiak, S. (2013) The Effects of Flanking Sequences in the Interaction of Polyglutamine Peptides with a Membrane Bilayer, The Journal of Physical Chemistry $B$.

(38) Darnell, G. D., Derryberry, J., Kurutz, J. W., and Meredith, S. C. (2009) Mechanism of Cis-Inhibition of PolyQ Fibrillation by PolyP: PPII Oligomers and the Hydrophobic Effect, Biophysical Journal 97, 2295-2305.

(39) Lanning, J. D., Hawk, A. J., Derryberry, J., and Meredith, S. C. (2010) Chaperone-like N-Methyl Peptide Inhibitors of Polyglutamine Aggregation, Biochemistry 49, 7108-7118.

(40) Monsellier, E., Redeker, V., Ruiz-Arlandis, G., Bousset, L., and Melki, R. (2015) Molecular Interaction between the Chaperone Hsc70 and the Nterminal Flank of Huntingtin Exon 1 Modulates Aggregation, Journal of Biological Chemistry 290, 2560-2576.

(41) Tam, S., Spiess, C., Auyeung, W., Joachimiak, L., Chen, B., Poirier, M. A., and Frydman, J. (2009) The chaperonin TRiC blocks a huntingtin sequence element that promotes the conformational switch to aggregation, Nature Structural \& Molecular Biology 16, 1279-U1298.

(42) Thompson, L. M., Aiken, C. T., Kaltenbach, L. S., Agrawal, N., Illes, K., Khoshnan, A., Martinez-Vincente, M., Arrasate, M., O'Rourke, J. G., Khashwji, H., Lukacsovich, T., Zhu, Y. Z., Lau, A. L., Massey, A., Hayden, M. R., Zeitlin, S. O., Finkbeiner, S., Green, K. N., LaFerla, F. M., Bates, G., Huang, L., Patterson, P. H., Lo, D. C., Cuervo, A. M., Marsh, J. L., and Steffan, J. S. (2009) IKK phosphorylates Huntingtin and targets it for degradation by the proteasome and lysosome, Journal of Cell Biology 187, 1083-1099.

(43) Schilling, B., Gafni, J., Torcassi, C., Cong, X., Row, R. H., LaFevre-Bernt, M. A., Cusack, M. P., Ratovitski, T., Hirschhorn, R., Ross, C. A., Gibson, B. W., and Ellerby, L. M. (2006) Huntingtin phosphorylation sites mapped by mass spectrometry - Modulation of cleavage and toxicity, Journal of Biological Chemistry 281, 23686-23697.

(44) Rand, K. D., Pringle, S. D., Morris, M., and Brown, J. M. (2012) SiteSpecific Analysis of Gas-Phase Hydrogen/Deuterium Exchange of Peptides and Proteins by Electron Transfer Dissociation, Analytical Chemistry 84, 1931-1940. 
(45) Kelley, N. W., Huang, X. H., Tam, S., Spiess, C., Frydman, J., and Pande, V. S. (2009) The Predicted Structure of the Headpiece of the Huntingtin Protein and Its Implications on Huntingtin Aggregation, Journal of Molecular Biology 388, 919-927. 


\section{Outlook: Physiologically relevant post-translational modifications of $\mathrm{Nt17}$ and regulation through complexation}

The work described in this document begins with identification of critical residues in htt exon 1, moves into modification of residues and measurements of gas phase structure, then finally, into Nt17-regulating effects of an endogenous sequence. What has not been discussed, however, is the effect of physiologically relevant post-translational modification. The work-in-progress described here aims to study acetylation and phosphorylation of $\mathrm{Nt} 17$ in the context of secondary structure, aggregation suppression, and other physiological properties, such as lipid binding. These studies will serve to supplement the works described in this document, and provide additional critical insight into mechanisms of Nt17mediated aggregation.

\subsection{Work in Progress: Acetlyation of $\mathrm{Nt}_{17 Q_{35}} \mathrm{P}_{10} \mathrm{KK}$ by sulfo-NHSA}

\subsubsection{Acetylation of Nt17 suppresses aggregate formation and alters aggregation} kinetics.

Nt17's ability to interact with substrates and other Nt17 tracts appears to be predicated on the ability of the sequence to form an amphipathic a-helix. As

shown in this work, and by others, ${ }^{1-3}$ structural perturbation by side chain modification at critical interfaces can partially absolve Nt17-mediated htt exon 1 multimer formation. The modifications in this work, however, are not physiological in nature. Thus, a thorough characterization of small, physiologically-relevant 
post-translational modifications is necessary. In Chapter 2, the N-terminal htt sequence was probed using HDX- and DHX-MS.
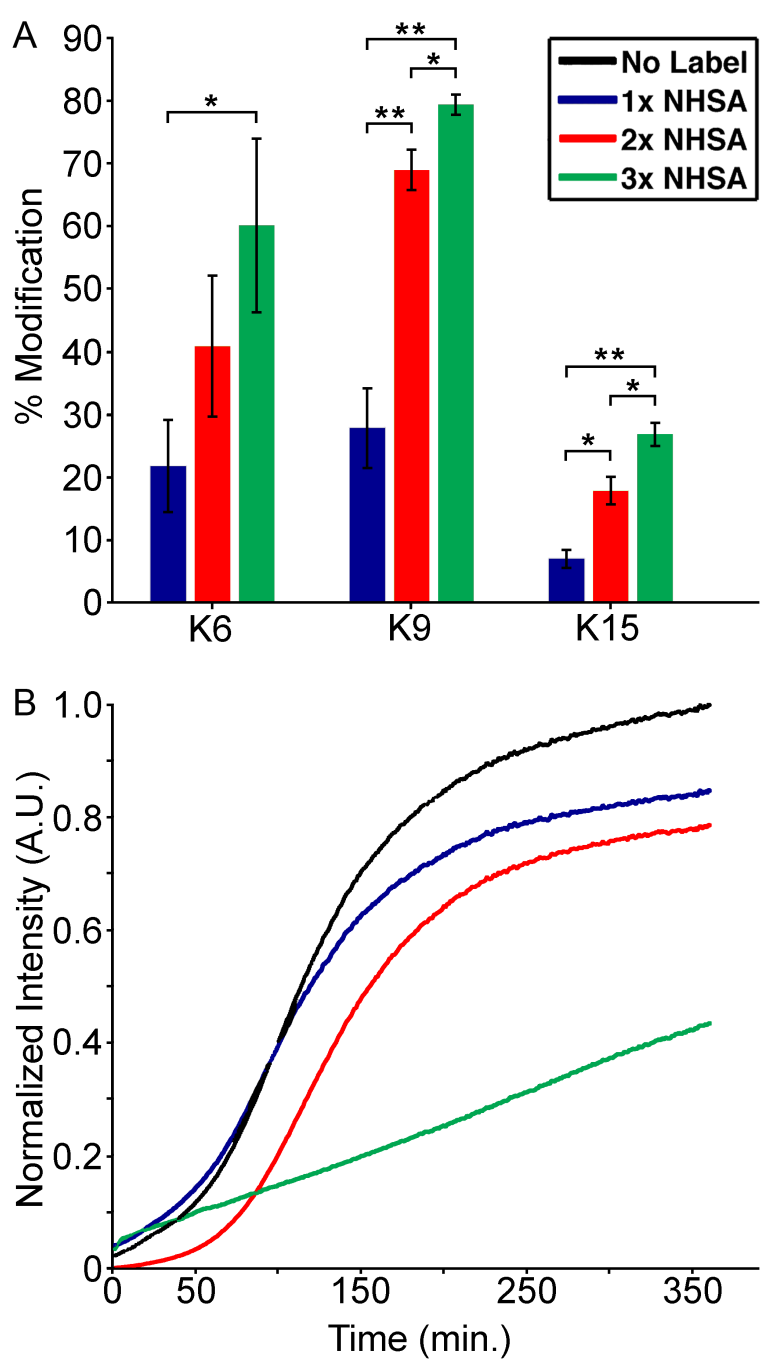

Figure 5.1. Acetylation of $N t 17 Q_{35} P_{10} K K$ reduces $\boldsymbol{\beta}$-sheet formation. A) Percent modification of each lysine residue in Nt17as a function of label concentration (mol:mol). B) ThT curves by label concentration. Increased labeling reduces $\beta$-sheet formation.
This study laid the groundwork for future investigations of lysine modifications by pinpointing critical lysine residues in $\mathrm{Nt17}$ that may be solvent-protected when incorporated in an aggregate. Lysine residues are targets for PTM's in vivo, thus, a characterization of the effects of these PTM's on aggregation kinetics and morphologies is a logical next step..

Nt17, in addition to its properties in driving aggregation, can undergo numerous PTM's, such as SUMOylation, ${ }^{4}$ phosphorylation, ${ }^{5,6}$ and acetylation. ${ }^{7}$ These PTM's can have significant effect on aggregation kinetics and morphologies. Preliminary data has been acquired that demonstrated the effect of acetylation on $\beta$ sheet formation (Figure 5.1). 
Lysine residues were modified with sulfo-N-hydroxysuccinimide (NHSA), which replaces primary amine groups with an acetyl group. Acetylation was chosen because it removes charge from the lysine side chain, thus preventing salt bridging to negatively charged residues. Modification by sulfo-NHSA of lysine $\varepsilon$-amino groups has been used as a structural probe in previous work. ${ }^{8,9}$ In those studies, the label was added as a probe to determine sites of surface availability. In the present work, however, NHSA is used to modify lysine residues in the monomeric state and determine implications on downstream aggregation. Labeling was performed with the molar ratios of NHSA to peptide of 1:1, 2:1, or 3:1. Controls were performed with no label added to the peptide. Percent modification of the three lysine residues in Nt17 was determined in a label concentration-dependent manner. The percent modification of the $\mathrm{N}$-terminus was not considered because it is pre-acetylated in the synthetic peptide. Of the three lysine residues in Nt17, $\mathrm{K} 9$ is most readily acetylated, while $\mathrm{K} 15$ does not appear to appreciably label relative to the other lysine residues. This result is consistent with previous findings that state $\mathrm{K} 9$ is an acetylation site in full-length huntingtin, which targets degradation to the proteasome. ${ }^{10,11} \mathrm{~K} 6$ and $\mathrm{K} 9$ are labeled appreciably more than $\mathrm{K} 15$ at the $1 \mathrm{x}$ concentration. At the $2 \mathrm{x}$ concentration of label, acetylation of $\mathrm{K} 9$ is nearly saturated, while $\mathrm{K} 6$ and $\mathrm{K} 15$ remain at 40 and $20 \%$ modified, respectively. Finally, at $3 x$ label:peptide concentration, modification at $\mathrm{K} 6$ did not appreciably increase from $2 \mathrm{x}$, while $\mathrm{K} 9$ and K15 modification is only increased slightly. 
Aggregation at each label:peptide ratio was monitored by ThT fluorescence (Figure 5.1B). While the lag phase of aggregation was not appreciably altered with exposure to $1 \mathrm{x}$ label, a decrease in fluorescence magnitude, and thus $\beta$-sheet formation, was observed. K6 and K9 were most readily acetylated, which is in good agreement with the data presented in Chapters 2 and 3 . At the $2 x$ label:peptide ratio, the increase in lysine modification results in an elongation of the aggregation lag phase, as well as another decrease in $\beta$-sheet formation. A significant increase in percent label from $1 \mathrm{x}$ to $2 x$ is observed for $\mathrm{K} 9$ and $\mathrm{K} 15$. Finally, at the $3 \mathrm{x}$ concentration, $\beta$-sheet formation is reduced by $50 \%$, relative to the $1 \times$ concentration at the 350 minute time point, which correlates to the statistically significant labeling increase from $1 x$ to $3 x$ for all lysine residues. Since K9 completely back-exchanges in deuterium exchange experiments, the large drop in $\beta$-sheet content is most likely attributed to labeling at K6 and K15. Still, modification at K9 increases significantly for all three label:peptide ratios, so its role cannot be completely disregarded. Further study is required to discern the actual role of each lysine residue. The most logical probe is a chemical point mutation that simulates acetylation ( $K$ to $Q$ ).

\subsection{Work in Progress: $\mathrm{K}$ to $\mathrm{Q}$ mutations in Nt17}

\subsubsection{K to Q mutations alter Nt17 secondary structure.}

A significant portion of the work described in this work focuses on structural ramifications of altering native Nt17 at critical lysine residues. Figure 5.2 shows a mass spectrum (A) and IMS-MS distributions of a doubly charged 

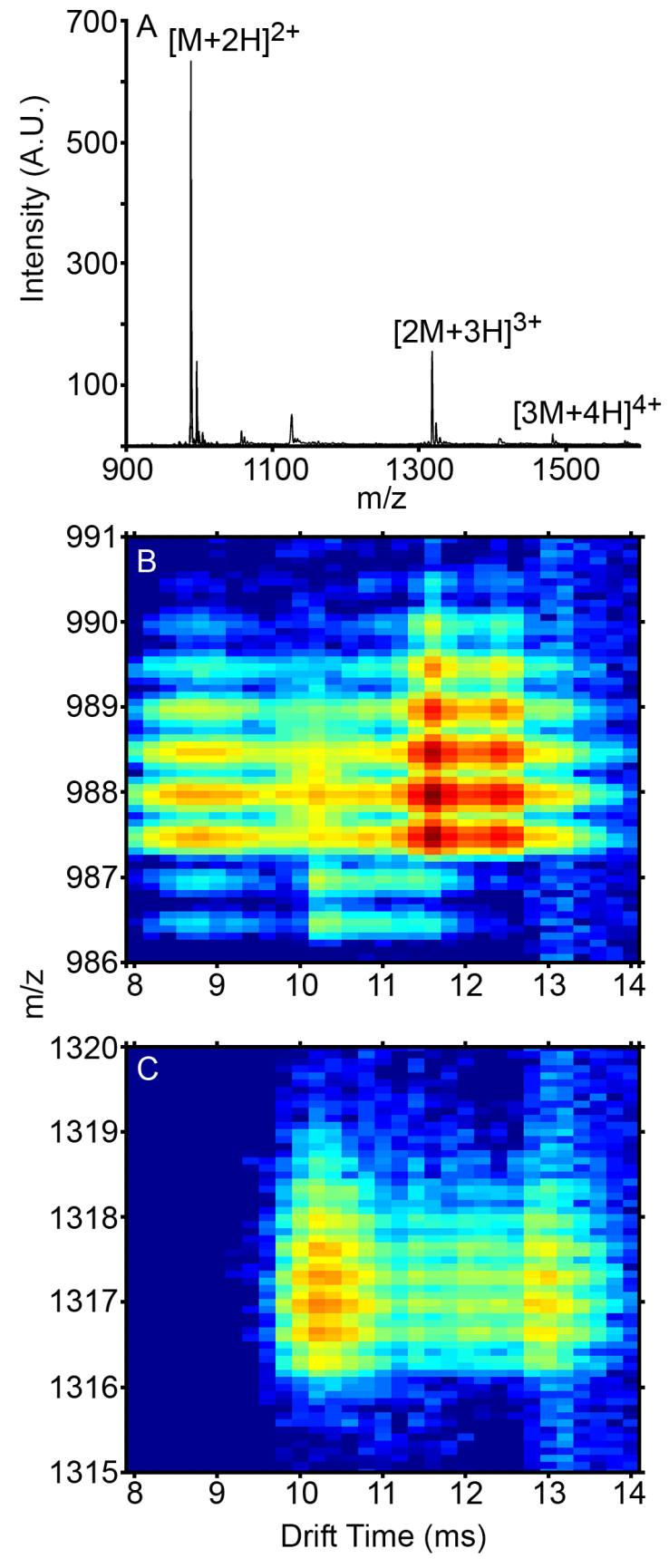

Figure 5.2. MS and IMS-MS distributions of Nt17 K6Q point mutations. A) Full mass spectrum of K6Q Nt17. lons of interest are labeled. B) IMS-MS distribution of the doubly charged monomer. C) IMS-MS distribution of the triply charged dimer.
K6Q Nt17 monomer (B) and dimer

(C). Peptides were prepared, and

IMS-MS experiments were

performed, in the same manner as

outlined in Chapters 3 and 4. A

decrease in overall multimer is

observed; however, dimer and

trimer species are not completely

abolished. Doubly charged

monomer dominates the mass

spectrum, which is to be expected

based on experiments with this

peptide

discussed in Chapters 3 and 4 . It

appears that monomer structure is affected by the $\mathrm{K} 6 \mathrm{Q}$ mutation. The same collapsed monomer feature

(Figure 5.2B, $11.6 \mathrm{~ms}$ ) is observed in this distribution, as was in others presented in previous chapters.

Interestingly, there appears to be a higher abundance of a more collapsed structure $(8.6 \mathrm{~ms})$. This could be due to a complete loss of structure 
from the helical state, though this has not been confirmed by MDS results. A more elongated conformation is also evident, which, strikingly, matches the drift time for the partially collapsed intermediate observed in Chapter 3. While acetylation is not the same as carbethoxylation, substitution of the primary amine group on K6 with a more nonpolar moiety could yield the same results as carbethoxylation, though not as dramatic.

The effect of acetylation could also be explored in the context of lipid binding. Nt17 and the polyP tract are required in the oligomerization of htt exon 1 on lipid substrates, ${ }^{12}$ thus interactions of $\mathrm{Nt} 17$ and lipid substrates could be of great interest in HD remediation. At first, simple colorimetric assays, such as a polydiacetlyene (PDA) assay ${ }^{13,14}$ could be used to determine Nt17 and pointmutated Nt17 lipid binding propensity. Experiments could then evolve into AFM experiments on a lipid bilayer that contains point-mutated htt exon 1 to determine extent of bilayer disruption. Finally, IMS-MS could supplement these experiments with gas-phase measurements and calculations of $\mathrm{Nt} 17$ structure featuring various point mutations.

\subsection{Future Direction: Targeting Nt17 with other proteins}

Physiologically endogenous proteins could also have a therapeutic effect in $\mathrm{HD}$ remediation. Hsc70 binds to the amino terminus of htt exon 1 and prevents aggregation by preventing helical homodimerization. ${ }^{15}$ To date, no work has focused on Hsc40/70 interactions with pre-aggregated species. If the $\mathrm{N}$-terminal region is indeed solvent-exposed in the early oligomeric or aggregated state, 
then theoretically the Hsc70 complex could function like the polyP-recognizing antibody, 3B5H10. 3B5H10 served to disaggregate htt exon 1 aggregates by taking advantage of the on-off equilibrium between monomer and fibril. ${ }^{16}$ The antibody sequestered soluble monomer from the amyloid fibril through association of the polyP region, and eventually, dissolved amyloid fibrils. The Hsc70 machine could take advantage of the same on/off equilibrium to recognize and sequester htt exon 1 through the $\mathrm{Nt} 17$ region. Intrabodies specific for $\mathrm{Nt} 17^{17}$, ${ }^{18}$ could be used in control experiments to compare morphological details between something that is specific for Nt17 and something that is less specific, in this case, the Hsc70 complex. Presence of secondary structure and residuespecific information could be obtained through HDX and DHX experiments, much like those performed in Chapter 2. This would only be a confirmatory study for the Nt17 intrabody controls; Nt17 adopts a helical structure in the intrabody complex. ${ }^{17}$ Whether Hsc70 acts on the unstructured or structured Nt17 remains to be shown, and could be demonstrated by the techniques outlined in Chapter 2 .

\subsection{Concluding remarks}

The works contained in this document are meant to provide a greater structural insight to HD-related aggregates, pinpoint critical residues and interfaces in early-stage aggregates, and develop a model for intrinsic, and possibly extrinsic, stabilization of a peptide sequence that is implicated in oligomer architecture. Additionally, these works detail the analytical utility of IMS- 
MS and gas-phase structural measurements in the study of relevant problems in

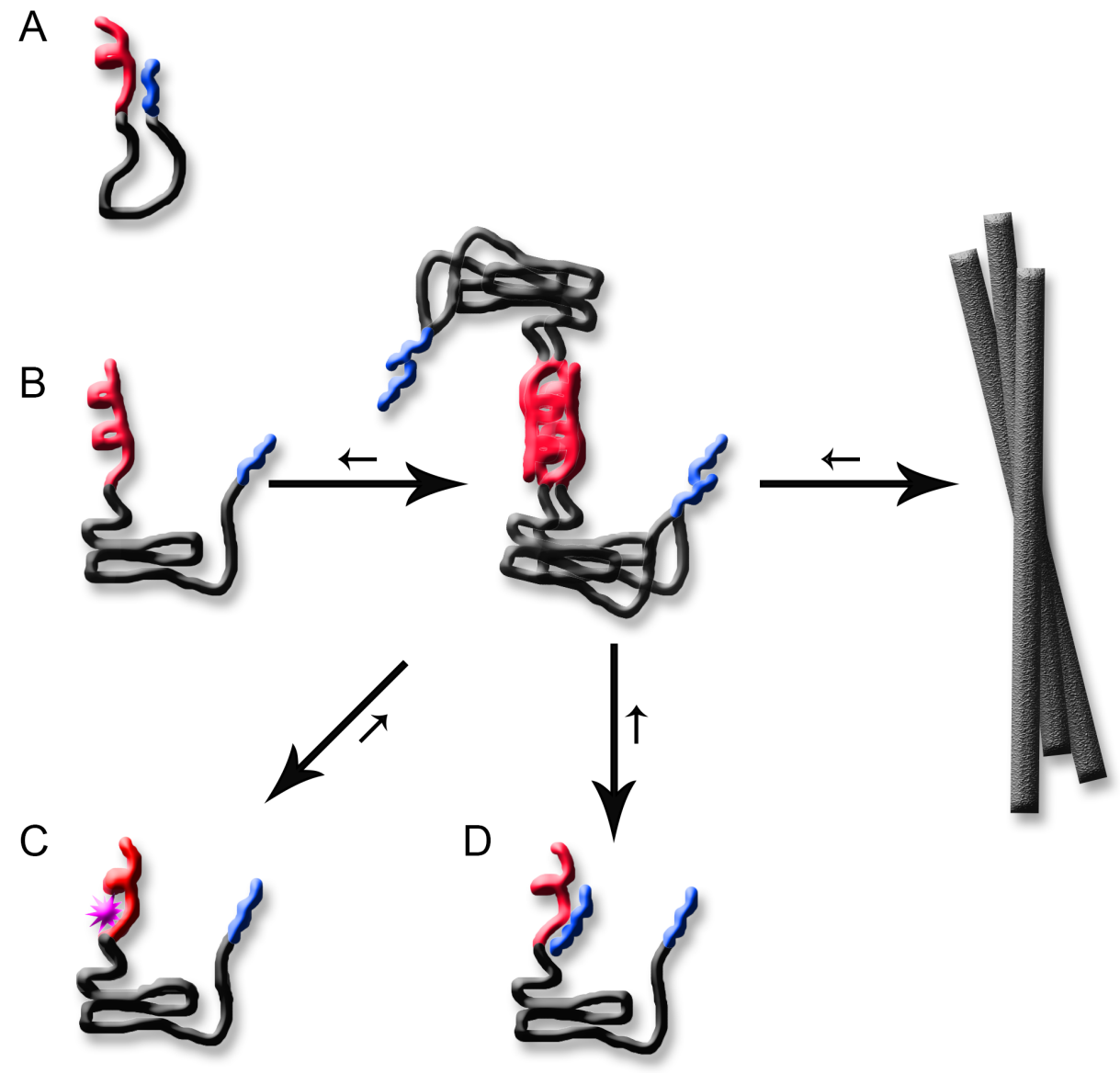

Figure 5.3. Schematic of Nt17-mediated htt exon 1 aggregation for modified and regulated Nt17. Nt17 association leading to aggregation is regulated by posttranslational modification and/or the presence of an external regulator. Red: Nt17; black: polyQ; blue: polyP. A) Normal, monomeric htt exon 1 with a non-disease-length polyQ. PolyP regulates Nt17 by not allowing helical transition. B) PolyQ gains rigidity at disease length, increasing distance from Nt17, thus allowing helical transition and association, to eventually form amyloid fibrils. C) A PTM in or near the helical sequence destabilizes helical character and moderates Nt17 association. D) Presence of an external regulator, such as polyP, prevents helical formation. In cases $C$ and D, amyloid fibrils still form, just not to the same extent as unmodified/unregulated Nt17. Additionally, it should be noted that this is only one part of an overall aggregation mechanism, as the polyQ region can still self-associate into amyloid fibrils.

biophysical chemistry. IMS-MS, when coupled to secondary techniques like

MDS, offers the benefit of discrete structural characterization of analytes that 
sample a heterogeneous mix of secondary, tertiary, and quaternary

arrangements. HDX and DHX supplement these studies and show, by deuterium retention in the condensed phase or deuterium incorporation in the gas phase, sites where labile heteroatoms are protected through $\mathrm{H}$-bond interactions or solvation. The condensed-phase experiments verified that $\mathrm{Nt} 17$ is solventexposed and mostly structured when incorporated in an aggregate. Additionally, the increased protection on $\mathrm{K} 6$ led to the hypothesis that the availability of lysine residues may be critical determinants in Nt17-mediated htt exon 1 aggregation. Subsequent ion mobility-mass spectrometry studies revealed structural heterogeneity in Nt17 that persists into the multimeric form; however, upon modification at two sites, one of which is $\mathrm{K} 6$, structure is perturbed and multimerization is drastically decreased. Finally, co-incubation of Nt17 with a polyP sequence drastically decreased helical content in the gas phase, and perhaps in a physiologically relevant construct, providing a structural framework for intrinsic htt exon 1 stabilization.

It is with this that these studies have laid the groundwork for future IMSMS studies to glean insight toward protein structure, with the eventual aim of ameliorating HD pathology. The next critical step is to further elucidate the effects of physiological post-translational modification in the condensed phase, the gas phase, and in the context of a cellular substrate. 


\subsection{References}

(1) Michalek, M., Salnikov, E. S., Werten, S., and Bechinger, B. (2013) Membrane Interactions of the Amphipathic Amino Terminus of Huntingtin, Biochemistry 52, 847-858.

(2) Jayaraman, M., Kodali, R., Sahoo, B., Thakur, A. K., Mayasundari, A., Mishra, R., Peterson, C. B., and Wetzel, R. (2012) Slow Amyloid Nucleation via alpha-Helix-Rich Oligomeric Intermediates in Short Polyglutamine-Containing Huntingtin Fragments, Journal of Molecular Biology 415, 881-899.

(3) Sivanandam, V. N., Jayaraman, M., Hoop, C. L., Kodali, R., Wetzel, R., and van der Wel, P. C. A. (2011) The Aggregation-Enhancing Huntingtin $\mathrm{N}$-Terminus Is Helical in Amyloid Fibrils, Journal of the American Chemical Society 133, 4558-4566.

(4) O'Rourke, J. G., Gareau, J. R., Ochaba, J., Song, W., Rasko, T., Reverter, D., Lee, J., Monteys, A. M., Pallos, J., Mee, L., Vashishtha, M., Apostol, B. L., Nicholson, T. P., Illes, K., Zhu, Y. Z., Dasso, M., Bates, G. P., Difiglia, M., Davidson, B., Wanker, E. E., Marsh, J. L., Lima, C. D., Steffan, J. S., and Thompson, L. M. (2013) SUMO-2 and PIAS1 Modulate Insoluble Mutant Huntingtin Protein Accumulation, Cell Reports 4, 362-375.

(5) Gu, X. F., Greiner, E. R., Mishra, R., Kodali, R., Osmand, A., Finkbeiner, S., Steffan, J. S., Thompson, L. M., Wetzel, R., and Yang, X. W. (2009) Serines 13 and 16 Are Critical Determinants of Full-Length Human Mutant Huntingtin Induced Disease Pathogenesis in HD Mice, Neuron 64, 828840.

(6) Aiken, C. T., Steffan, J. S., Guerrero, C. M., Khashwji, H., Lukacsovich, T., Simmons, D., Purcell, J. M., Menhaji, K., Zhu, Y. Z., Green, K., LaFerla, F., Huang, L., Thompson, L. M., and Marsh, J. L. (2009) Phosphorylation of Threonine 3 IMPLICATIONS FOR HUNTINGTIN AGGREGATION AND NEUROTOXICITY, Journal of Biological Chemistry 284, 29427-29436.

(7) Thompson, L. M., Aiken, C. T., Kaltenbach, L. S., Agrawal, N., Illes, K., Khoshnan, A., Martinez-Vincente, M., Arrasate, M., O'Rourke, J. G., Khashwji, H., Lukacsovich, T., Zhu, Y. Z., Lau, A. L., Massey, A., Hayden, M. R., Zeitlin, S. O., Finkbeiner, S., Green, K. N., LaFerla, F. M., Bates, G., Huang, L., Patterson, P. H., Lo, D. C., Cuervo, A. M., Marsh, J. L., and Steffan, J. S. (2009) IKK phosphorylates Huntingtin and targets it for degradation by the proteasome and lysosome, Journal of Cell Biology 187, 1083-1099.

(8) Mendoza, V. L., Baron-Rodriguez, M. A., Blanco, C., and Vachet, R. W. (2011) Structural Insights into the Pre-Amyloid Tetramer of beta-2Microglobulin from Covalent Labeling and Mass Spectrometry, Biochemistry 50, 6711-6722.

(9) Mendoza, V. L., Antwi, K., Baron-Rodriguez, M. A., Blanco, C., and Vachet, R. W. (2010) Structure of the Preamyloid Dimer of beta-2Microglobulin from Covalent Labeling and Mass Spectrometry, Biochemistry 49, 1522-1532. 
(10) Cong, X., Held, J. M., DeGiacomo, F., Bonner, A., Chen, J. M., Schilling, B., Czerwieniec, G. A., Gibson, B. W., and Ellerby, L. M. (2011) Mass Spectrometric Identification of Novel Lysine Acetylation Sites in Huntingtin, Molecular \& Cellular Proteomics 10.

(11) Jeong, H., Then, F., Melia Jr, T. J., Mazzulli, J. R., Cui, L., Savas, J. N., Voisine, C., Paganetti, P., Tanese, N., Hart, A. C., Yamamoto, A., and Krainc, D. (2009) Acetylation Targets Mutant Huntingtin to Autophagosomes for Degradation, Cell 137, 60-72.

(12) Burke, K. A., Kauffman, K. J., Umbaugh, C. S., Frey, S. L., and Legleiter, J. (2013) The Interaction of Polyglutamine Peptides With Lipid Membranes is Regulated by Flanking Sequences Associated with Huntingtin, Journal of Biological Chemistry.

(13) Zheng, F., Wu, Z., and Chen, Y. H. (2012) A quantitative method for the measurement of membrane affinity by polydiacetylene-based colorimetric assay, Analytical Biochemistry 420, 171-176.

(14) Evrard, D., Touitou, E., Kolusheva, S., Fishov, Y., and Jelinek, R. (2001) A new colorimetric assay for studying and rapid screening of membrane penetration enhancers, Pharmaceutical Research 18, 943-949.

(15) Monsellier, E., Redeker, V., Ruiz-Arlandis, G., Bousset, L., and Melki, R. (2015) Molecular Interaction between the Chaperone Hsc70 and the Nterminal Flank of Huntingtin Exon 1 Modulates Aggregation, Journal of Biological Chemistry 290, 2560-2576.

(16) Legleiter, J., Lotz, G. P., Miller, J., Ko, J., Ng, C., Williams, G. L., Finkbeiner, S., Patterson, P. H., and Muchowski, P. J. (2009) Monoclonal Antibodies Recognize Distinct Conformational Epitopes Formed by Polyglutamine in a Mutant Huntingtin Fragment, Journal of Biological Chemistry 284, 21647-21658.

(17) Colby, D. W., Garg, P., Holden, T., Chao, G., Webster, J. M., Messer, A., Ingram, V. M., and Wittrup, K. D. (2004) Development of a Human Light Chain Variable Domain (VL) Intracellular Antibody Specific for the Amino Terminus of Huntingtin via Yeast Surface Display, Journal of Molecular Biology 342, 901-912.

(18) Colby, D. W., Chu, Y., Cassady, J. P., Duennwald, M., Zazulak, H., Webster, J. M., Messer, A., Lindquist, S., Ingram, V. M., and Wittrup, K. D. (2004) Potent inhibition of huntingtin aggregation and cytotoxicity by a disulfide bond-free single-domain intracellular antibody, Proceedings of the National Academy of Sciences of the United States of America 101, 17616-17621. 
Ion mobility-mass spectrometry for structural characterization and applied 'omics: A study in neurodegenerative disease

James R. Arndt

Dissertation submitted to the Eberly College of Arts and Sciences at West Virginia University in partial fulfillment of the requirements for the degree of

Doctor of Philosophy in Chemistry

C. Eugene Bennett Department of Chemistry

APPROVAL OF THE EXAMINING COMMITTEE

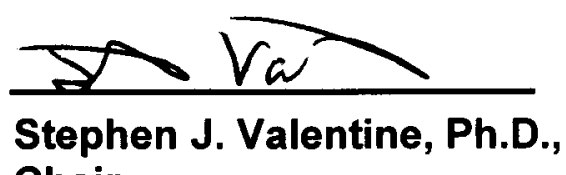
Chair
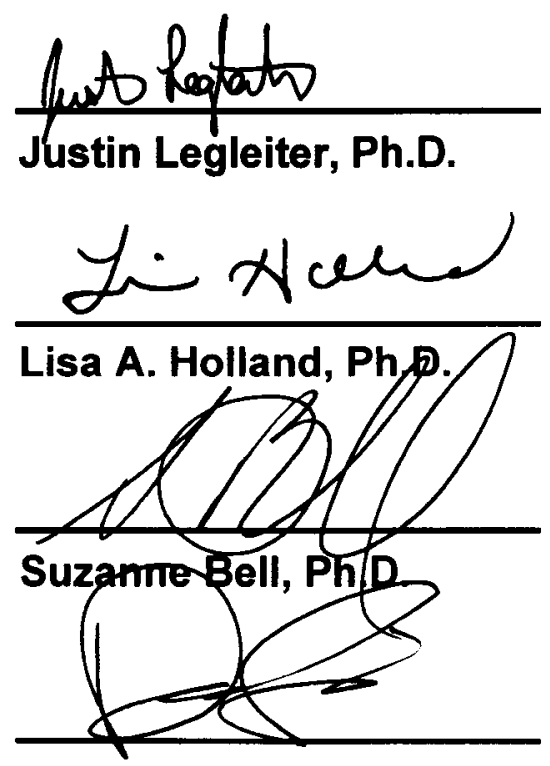

Date

David M. Smith, Ph.D. 